Supporting Information

\title{
Enantioselective Synthesis of the Polyhydroxylated Chain of Oscillariolide and Phormidolides A-C.
}

\begin{abstract}
Alejandro Gil, Janire Lamariano-Merketegi, Adriana Lorente, Fernando Albericio and Mercedes Álvarez

Barcelona Science Park-University of Barcelona, Baldiri Reixac 10, E-08028 Barcelona, Spain. CIBERBBN, Networking Centre on Bioengineering, Biomaterials and Nanomedicine, Department of Organic Chemistry, University of Barcelona, 08028 Barcelona, Spain; Laboratory of Organic Chemistry, Faculty of Pharmacy, University of Barcelona, 08028 Barcelona, Spain.
\end{abstract}

mercedesalvarez@ub.edu 


\section{TABLE OF CONTENTS}

$\begin{array}{ll}\text { 1. General Procedures } & \text { SI } 2\end{array}$

2. Experimental procedures and characterization $\quad$ SI $2-$ SI15

3. Solvent optimization in transformation $\mathbf{5} \rightarrow \mathbf{1 0}$ SI 15

$\begin{array}{lr}\text { 4. C23 Configuration of } \mathbf{1 0} \text { determination } & \text { SI } 16\end{array}$

5. Solvent and temperature optimization in transformation $\mathbf{1 0} \rightarrow$ SI4 $\quad$ SI 16

6. C19 Configuration of $\mathbf{1 3}$ determination $\quad$ SI 16

$\begin{array}{lr}\text { 7. C27 Configuration of } \mathbf{1 6} \text { determination } & \text { SI } 17\end{array}$

8. Table $1 . \mathrm{H}^{1}$ - and $\mathrm{C}^{13}$-NMR data of compound $\mathbf{1}$ and oscillariolide SI 18

9. Table 2. $\mathrm{H}^{1}$ - and $\mathrm{C}^{13}$-NMR data of compound 2 and phormidolide A SI 18

$\begin{array}{ll}\text { 10. NMR spectra of compounds } & \text { SI 19- SI53 }\end{array}$

11. References $\quad$ SI 54 


\section{General Procedures}

Tetrahydrofuran (THF) and $N, N$-dimethylformamide (DMF) were dried using a PureSolv solvent purification system. All other solvents and reagents were used as purchased without further purification, unless otherwise indicated. Flash column chromatography was performed on silica gel (60A $35-70 \mu \mathrm{m})$ as stationary phase. Analytical TLC was performed on pre-coated silica gel $60 \mathrm{~F}_{254}$ plates $(0.2 \mathrm{~mm}$ thick, $20 \times 20 \mathrm{~cm})$ and visualized under UV light (254 and $360 \mathrm{~nm}$ ), with anisaldehyde in conc. $\mathrm{H}_{2} \mathrm{SO}_{4}$ or with phosphomolybdic acid in ethanol. Polarimetry studies were performed on a Perkin-Elmer 241 or JascoP-2000 polarimeter equipped with a Na-lamp. IR spectra were recorded on a Varian Mercury $400 \mathrm{MHz}$ or a Varian VNMRS500 500MHz. Chemical shifts are reported in ppm referenced to the appropriate residual solvent peaks $\left(\mathrm{CDCl}_{3}\right)$ and coupling constants are reported in $\mathrm{Hz}$. Multiplicity of the carbons was assigned with gHSQC experiments. Standard abbreviations for off-resonance decoupling were employed: $\mathrm{s}=$ singlet, $\mathrm{d}=$ doublet, $\mathrm{t}=$ triplet, $\mathrm{q}=$ quadruplet, $\mathrm{bs}=$ broad singlet, $\mathrm{bd}=$ broad doublet, $\mathrm{m}=$ multiplet. The same abbreviations were also used for the multiplicity of signals in ${ }^{1} \mathrm{H}-\mathrm{NMR}$. High Resolution Mass Spectroscopy (HRMS) was performed an Agilent LC/MSD-TOF 2006 system using the ESI-MS technique.

\section{Experimental procedures and characterization}

(4R)-4-Benzyl-3-((2R)-2-((4S)-2-(4-methoxyphenyl)-1,3-dioxan-4-yl)propanoyl)oxazolidin2-one (8)<smiles>[Y6]C1OCC[C@H]([C@H](C)C(=O)N2C(=O)OC[C@H]2Cc2ccccc2)O1</smiles>

DDQ (6.9 g, $30.3 \mathrm{mmol})$ was added to a solution of aldol $7^{1}(11.8 \mathrm{~g}$, $27.6 \mathrm{mmol})$ in dry $\mathrm{CH}_{2} \mathrm{Cl}_{2}(600 \mathrm{~mL})$ and the reaction was stirred for 45 minutes. After this time, the reaction was filtered through a pad of celite. The solution was washed three times with $\mathrm{NaHCO}_{3}$ and the organic layer was dried over $\mathrm{Na}_{2} \mathrm{SO}_{4}$, filtered and the solvent was removed under reduced pressure. Purification by silica gel column chromatography with hexane-EtOAc (9:1) yielded $\mathbf{8}(11.08 \mathrm{~g}, 90 \%)$ as a colorless oil. $[\alpha]_{\mathrm{D}}=-34.6\left(\mathrm{c} 1.0, \mathrm{CH}_{2} \mathrm{Cl}_{2}\right)$. IR $\left(\mathrm{KBr}\right.$ film) $v 3028,2968,2858,1777,1693,1455,1382,1248,1109 \mathrm{~cm}^{-1} .{ }^{1} \mathrm{H}-\mathrm{NMR}(400 \mathrm{MHz}$, $\left.\mathrm{CDCl}_{3}\right): \delta 1.34(\mathrm{~d}, J=6.5 \mathrm{~Hz}, 3 \mathrm{H}), 1.59-1.62(\mathrm{~m}, 1 \mathrm{H}), 1.83-1.95(\mathrm{~m}, 1 \mathrm{H}), 2.77(\mathrm{dd}, J=13.4$, $9.6 \mathrm{~Hz}, 1 \mathrm{H}), 3.28(\mathrm{dd}, J=13.4,3.4 \mathrm{~Hz}, 1 \mathrm{H}), 3.79$ (s, 3H), 3.95 (ddd, $J=12.3,11.5,2.6 \mathrm{~Hz}, 1 \mathrm{H}$ ), $4.07-4.17(\mathrm{~m}, 4 \mathrm{H}), 4.20-4.28(\mathrm{~m}, 1 \mathrm{H}), 4.62-4.69(\mathrm{~m}, 1 \mathrm{H}), 5.49(\mathrm{~s}, 1 \mathrm{H}), 6.84-6.91(\mathrm{~m}$, $2 \mathrm{H}), 7.18-7.23(\mathrm{~m}, 2 \mathrm{H}), 7.27-7.42(\mathrm{~m}, 5 \mathrm{H}) .{ }^{13} \mathrm{C}-\mathrm{NMR}\left(100.6 \mathrm{MHz}, \mathrm{CDCl}_{3}\right): \delta 13.1(\mathrm{q}), 28.3$ $(\mathrm{t}), 37.9(\mathrm{t}), 42.3(\mathrm{~d}), 55.3(\mathrm{~d}), 55.5(\mathrm{q}), 66.0(\mathrm{t}), 66.7$ (t), $77.6(\mathrm{~d}), 100.9$ (d), $113.5(2)(\mathrm{d}), 127.2$ (2) (d), 127.4 (d), 128.9 (d), 129.4 (d), 131.2 (s), 135.2 (s), 153.1 (s), 159.8 (s), 174.4 (s). HRMS (ESI+): $m / z$ calculated for $\mathrm{C}_{24} \mathrm{H}_{28} \mathrm{NO}_{6}[\mathrm{M}+\mathrm{H}]^{+} 426.1911$, found 426.1923 . 
(2S)-2-((4S)-2-(4-Methoxyphenyl)-1,3-dioxan-4-yl)propan-1-ol (S1)<smiles>[Y6]C1OCC[C@H]([C@@H](C)CO)O1</smiles>

A $2 \mathrm{M}$ solution of $\mathrm{LiBH}_{4}$ in THF $(13.53 \mathrm{~mL}, 27.1 \mathrm{mmol})$ was added to a solution of $8(5.75 \mathrm{~g}, 13.53 \mathrm{mmol})$ in $\mathrm{Et}_{2} \mathrm{O}(210 \mathrm{~mL})$ and $\mathrm{MeOH}(2.1 \mathrm{~mL})$ at $0^{\circ} \mathrm{C}$ during 30 minutes and the mixture was stirred at $0{ }^{\circ} \mathrm{C}$ for 30 minutes more. The reaction was quenched by addition of saturated solution of $\mathrm{NH}_{4} \mathrm{Cl}$ and extracted three times with $\mathrm{Et}_{2} \mathrm{O}$. The organic phase was dried over $\mathrm{Na}_{2} \mathrm{SO}_{4}$, filtered and the solvent was removed under reduced pressure. Purification by silica gel column chromatography with hexane-EtOAc (1:1) yielded $\mathbf{S 1}(2.8 \mathrm{~g}, 82 \%)$ as a colorless oil. $[\alpha]_{\mathrm{D}}=+13.9$ (c 1.0, $\mathrm{CH}_{2} \mathrm{Cl}_{2}$ ). IR (KBr film) v 3436 (b), 2962, 2858, 1614, 1517, 1395, 1249, $1103 \mathrm{~cm}^{-1} .{ }^{1} \mathrm{H}-\mathrm{NMR}$ $\left(400 \mathrm{MHz}, \mathrm{CDCl}_{3}\right): \delta 1.00(\mathrm{~d}, J=7.1 \mathrm{~Hz}, 3 \mathrm{H}), 1.40-1.46(\mathrm{~m}, 1 \mathrm{H}), 1.93-1.99(\mathrm{~m}, 1 \mathrm{H}), 2.00-$ $2.07(\mathrm{~m}, 1 \mathrm{H}), 3.59-3.65(\mathrm{~m}, 1 \mathrm{H}), 3.74(\mathrm{~d}, J=7.7 \mathrm{~Hz}, 1 \mathrm{H}), 3.80(\mathrm{~s}, 3 \mathrm{H}), 3.96(\mathrm{ddd}, J=12.2$, $11.4,2.5 \mathrm{~Hz}, 1 \mathrm{H}$ ), 4.02 (ddd, $J=11.6,4.2,2.4 \mathrm{~Hz}, 1 \mathrm{H}), 4.29$ (ddd, $J=11.4,5.0,1.5 \mathrm{~Hz}, 1 \mathrm{H}$ ), $5.47(\mathrm{~s}, 1 \mathrm{H}), 6.85-6.90(\mathrm{~m}, 2 \mathrm{H}), 7.35-7.40(\mathrm{~m}, 2 \mathrm{H}) .{ }^{13} \mathrm{C}-\mathrm{NMR}\left(100.6 \mathrm{MHz}, \mathrm{CDCl}_{3}\right): \delta 11.7$ (q), $27.1(\mathrm{t}), 39.4$ (d), 55.3 (q), 65.5 (t), 67.1 (t), 79.7 (d), 101.3 (d), 113.6 (d), 127.2 (d), 131.2 (s), 159.9 (s). HRMS (ESI+): $m / z$ calculated for $\mathrm{C}_{14} \mathrm{H}_{21} \mathrm{O}_{4}[\mathrm{M}+\mathrm{H}]^{+}$253.1437, found 253.1434 .

\section{(2R)-2-((4S)-2-(4-Methoxyphenyl)-1,3-dioxan-4-yl)propanal (5)}<smiles>[R16]C1OCC[C@H]([C@H](C)C=O)O1</smiles>

$\mathrm{NaHCO}_{3}(370 \mathrm{mg}, 4.4 \mathrm{mmol})$ and DMP $(930 \mathrm{mg}, 2.2 \mathrm{mmol})$ were added to a solution of alcohol $\mathbf{S 1}(462 \mathrm{mg}, 1.83 \mathrm{mmol})$ in $\mathrm{CH}_{2} \mathrm{Cl}_{2}(40 \mathrm{~mL})$ and the solution was stirred for $1 \mathrm{~h}$. The reaction mixture was diluted with a saturated solution of $\mathrm{Na}_{2} \mathrm{~S}_{2} \mathrm{O}_{3}$ and a saturated solution of $\mathrm{NaHCO}_{3}$ and the residue was extracted with $\mathrm{CH}_{2} \mathrm{Cl}_{2}$. The organic extracts were dried over $\mathrm{Na}_{2} \mathrm{SO}_{4}$, filtered and concentrated under reduced pressure. Purification by silica gel column chromatography with hexane-EtOAc (9:1) yielded aldehyde $5(398 \mathrm{mg}, 91 \%)$ as a colorless oil. $[\alpha]_{\mathrm{D}}=-10.9(\mathrm{c} 1.0$, $\left.\mathrm{CH}_{2} \mathrm{Cl}_{2}\right)$. IR $\left(\mathrm{KBr}\right.$ film) v 2964, 2839, 1726, 1615, 1518, 1393, 1249, $1102 \mathrm{~cm}^{-1} .{ }^{1} \mathrm{H}-\mathrm{NMR}$ (400 $\left.\mathrm{MHz}, \mathrm{CDCl}_{3}\right): \delta 1.21(\mathrm{~d}, J=7.1 \mathrm{~Hz}, 3 \mathrm{H}), 1.52-1.57(\mathrm{~m}, 1 \mathrm{H}), 1.85-1.99(\mathrm{~m}, 1 \mathrm{H}), 2.57-2.66$ (m, 1H), 3.80 (s, 3H), $3.92-4.04(\mathrm{~m}, 1 \mathrm{H}), 4.20-4.25$ (m, 1H), 4.28 (ddd, $J=11.5,5.1,1.5 \mathrm{~Hz}$, 1H), $5.50(\mathrm{~s}, 1 \mathrm{H}), 6.83-6.91(\mathrm{~m}, 2 \mathrm{H}), 7.31-7.43(\mathrm{~m}, 2 \mathrm{H}), 9.83(\mathrm{~d}, J=1.2 \mathrm{~Hz}, 1 \mathrm{H}) .{ }^{13} \mathrm{C}-\mathrm{NMR}$ $\left(100.6 \mathrm{MHz}, \mathrm{CDCl}_{3}\right):{ }^{13} \mathrm{C} \mathrm{NMR}\left(101 \mathrm{MHz}, \mathrm{CDCl}_{3}\right) \delta 8.8(\mathrm{q}), 28.3(\mathrm{t}), 50.8(\mathrm{~d}), 55.3(\mathrm{q}), 66.7$ (t), 76.5 (d), 101.2 (d), 113.6 (d), 127.3 (d), 130.9 (s), 159.9 (s), 203.6 (d). HRMS (ESI+): m/z calculated for $\mathrm{C}_{14} \mathrm{H}_{19} \mathrm{O}_{4}[\mathrm{M}+\mathrm{H}]^{+} 251.1278$, found 251.1282. 
(5R,6S)-1-((tert-Butyldimethylsilyl)oxy)-5-hyd roxy-6-((4S)-2-(4-methoxyphenyl)-1,3dioxan-4-yl)-2,2-dimethylheptan-3-one (10)

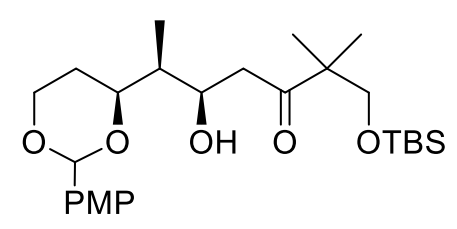

A solution of silylenol ether $9^{2}(1.4 \mathrm{~g}, 4.71 \mathrm{mmol})$ in $\mathrm{CH}_{2} \mathrm{Cl}_{2}: \mathrm{Et}_{2} \mathrm{O}(95: 5,5 \mathrm{~mL})$ was added to a cooled solution ($\left.78^{\circ} \mathrm{C}\right)$ of aldehyde $5(1.18 \mathrm{~g}, 4.71 \mathrm{mmol})$ in $\mathrm{CH}_{2} \mathrm{Cl}_{2}: \mathrm{Et}_{2} \mathrm{O}(95: 5$, $50 \mathrm{~mL}) . \mathrm{BF}_{3} \cdot \mathrm{OEt}_{2}(0.58 \mathrm{~mL}, 4.71 \mathrm{mmol})$ was added via syringe pump with a rate of $0.25 \mathrm{mmol} / \mathrm{min}$. The reaction was stirred at $-78^{\circ} \mathrm{C}$ for $2 \mathrm{~h}$ and quenched with saturated solution of $\mathrm{NaHCO}_{3}(50 \mathrm{~mL})$. The residue was extracted three times with $\mathrm{CH}_{2} \mathrm{Cl}_{2}$, dried over $\mathrm{Na}_{2} \mathrm{SO}_{4}$, filtrated and concentrated under reduced pressure. Purification by silica gel column cromatography with hexane-EtOAc (8:2) affords $1.7 \mathrm{~g}$ of $\mathbf{1 0}$ and $33 \mathrm{mg}$ of $\mathbf{1 0 ( 2 3 S )}$ (98 $\%$ based in the recovery of 5) as a separable mixture of diasteromers and $297 \mathrm{mg}$ of $\mathbf{5}$ as a recovered starting material. $[\alpha]_{\mathrm{D}}=+14.5\left(\mathrm{c} 1.0, \mathrm{CH}_{2} \mathrm{Cl}_{2}\right)$. IR $(\mathrm{KBr}$ film) $\vee 3528,2956,2857$, $1699,1615,1517,1393,1249,1105 \mathrm{~cm}^{-1} .{ }^{1} \mathrm{H}$ NMR $\left(400 \mathrm{MHz}, \mathrm{CDCl}_{3}\right) \delta 0.01(\mathrm{~d}, J=1.5 \mathrm{~Hz}$, 6H), $0.86(\mathrm{~s}, 9 \mathrm{H}), 1.04(\mathrm{~d}, J=7.0 \mathrm{~Hz}, 3 \mathrm{H}), 1.09$ (d, $J=4.8 \mathrm{~Hz}, 6 \mathrm{H}), 1.52-1.57(\mathrm{~m}, 1 \mathrm{H}), 1.63-$ $1.72(\mathrm{~m}, 1 \mathrm{H}), 1.88-2.00(\mathrm{~m}, 1 \mathrm{H}), 2.66-2.79(\mathrm{~m}, 2 \mathrm{H}), 3.56(\mathrm{~d}, J=1.4 \mathrm{~Hz}, 2 \mathrm{H}), 3.80(\mathrm{~s}, 3 \mathrm{H})$, 3.97 (ddd, $J=12.3,11.4,2.6 \mathrm{~Hz}, 1 \mathrm{H}), 4.03(\mathrm{ddd}, J=11.5,5.2,2.4 \mathrm{~Hz}, 1 \mathrm{H}), 4.22-4.29$ (m, $1 \mathrm{H}), 4.25-4.32(\mathrm{~m}, 1 \mathrm{H}), 5.49(\mathrm{~s}, 1 \mathrm{H}), 6.84-6.90(\mathrm{~m}, 2 \mathrm{H}), 7.34-7.42(\mathrm{~m}, 2 \mathrm{H}) .{ }^{13} \mathrm{C}$ NMR $(101$ $\left.\mathrm{MHz}, \mathrm{CDCl}_{3}\right) \delta$-5.5 (q), $8.9(\mathrm{q}), 18.3(\mathrm{~s}), 21.5(\mathrm{q}), 25.9$ (q), 28.8 (t), $42.2(\mathrm{~d}), 42.6(\mathrm{t}), 49.9(\mathrm{~s})$, $55.4(\mathrm{q}), 67.3(\mathrm{t}), 68.8$ (d), 70.4 (t), 79.5 (d), 101.1 (d), 113.7 (d), 127.4 (d), 131.6 (s), 160.0 (s), 216.6 (s). HRMS (ESI+): $\mathrm{m} / z$ calculated for $\mathrm{C}_{26} \mathrm{H}_{44} \mathrm{NaO}_{6} \mathrm{Si}[\mathrm{M}+\mathrm{Na}]^{+}$503.2799, found 503.2802.

(5S,6S)-1-((tert-Butyldimethylsilyl)oxy)-5-hydroxy-6-((4S)-2-(4-methoxyphenyl)-1,3dioxan-4-yl)-2,2-dimethylheptan-3-one (10(23S))

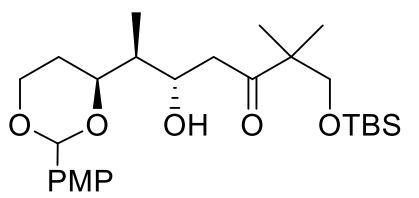

${ }^{1} \mathrm{H}-\mathrm{NMR}\left(400 \mathrm{MHz}, \mathrm{CDCl}_{3}\right): \delta 0.02(\mathrm{~d}, J=1.9 \mathrm{~Hz}, 6 \mathrm{H}), 0.86(\mathrm{~s}$, $9 \mathrm{H}), 0.99$ (d, $J=6.9 \mathrm{~Hz}, 3 \mathrm{H}), 1.09$ (s, 6H), $1.56-1.62(\mathrm{~m}, 3 \mathrm{H})$,

$1.66(\mathrm{dt}, J=6.9,2.3 \mathrm{~Hz}, 1 \mathrm{H}), 1.94-2.03(\mathrm{~m}, 1 \mathrm{H}), 2.68(\mathrm{dd}, J=$ $17.8,6.9 \mathrm{~Hz}, 1 \mathrm{H}), 2.94(\mathrm{dd}, J=17.8,6.1 \mathrm{~Hz}, 1 \mathrm{H}), 3.54(\mathrm{~d}, J=9.8 \mathrm{~Hz}, 1 \mathrm{H}), 3.61(\mathrm{~d}, J=9.8 \mathrm{~Hz}$, 1H), 3.79 (s, 3H), $3.81-3.87(\mathrm{~m}, 2 \mathrm{H}), 4.16(\mathrm{dt}, J=10.0,2.3 \mathrm{~Hz}, 1 \mathrm{H}), 4.45(\mathrm{td}, J=6.5,2.3 \mathrm{~Hz}$, $1 \mathrm{H}), 5.56(\mathrm{~s}, 1 \mathrm{H}), 6.77-6.95(\mathrm{~m}, 2 \mathrm{H}), 7.30-7.44(\mathrm{~m}, 2 \mathrm{H}) .{ }^{13} \mathrm{C} \mathrm{NMR}\left(101 \mathrm{MHz}, \mathrm{CDCl}_{3}\right) \delta-5.6$ (q), $6.4(\mathrm{q}), 18.2(\mathrm{~s}), 21.4(\mathrm{q}), 25.8(\mathrm{q}), 34.5(\mathrm{~d}), 35.3(\mathrm{t}), 40.7(\mathrm{t}), 49.7(\mathrm{~s}), 55.3(\mathrm{q}), 61.2(\mathrm{t})$, 70.0 (t), 76.9 (d), 80.2 (d), 101.6 (d), 113.6 (d), 127.4 (d), 131.1 (s), 159.9 (s), 212.3 (s).

\section{General procedure for derivatization with methoxyphenylacetic acid (MPA):}

$\alpha$-Methoxyphenylacetic acid (5 eq.) and $\mathrm{EDC} \cdot \mathrm{HCl}$ (5 eq.) were added to a solution of alcohol 10(5R) (1 eq.) in $\mathrm{CH}_{2} \mathrm{Cl}_{2}$, then DMAP (0.1 eq.) was added and the solution was stirred for $1 \mathrm{~h}$. The solution was filtered through celite ${ }^{\circledR} 545$, poured into $\mathrm{Et}_{2} \mathrm{O}$ and washed with $0.2 \mathrm{M}$ aqueous $\mathrm{HCl}$. The organic residue was dried over $\mathrm{Na}_{2} \mathrm{SO}_{4}$, filtered and the solvent was removed under 
reduced pressure. Purification by silica gel column chromatography with hexane-EtOAc (90:10) yielded the corresponding esters $\mathbf{S} \mathbf{2}$ and $\mathbf{S 3}$ as colorless oils.

(2R,3R)-7-((tert-Butyldimethylsilyl)oxy)-2-((4S)-2-(4-methoxyphenyl)-1,3-dioxan-4-yl)-6,6dimethyl-5-oxoheptan-3-yl (2R)-2-methoxy-2-phenylacetate (S2)

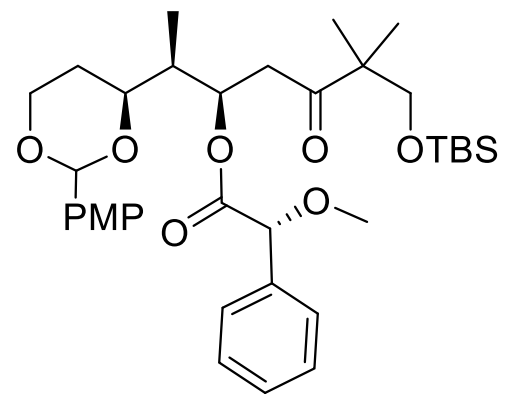

Aldol $10(20 \mathrm{mg}, 0.04 \mathrm{mmol})$ and $(R)$-MPA (35 mg, 0.21 mmol) afforded MPA derivative $\mathbf{S 2}(22 \mathrm{mg}, 82 \%) .{ }^{1} \mathrm{H}$ NMR $\left(400 \mathrm{MHz}, \mathrm{CDCl}_{3}\right) \delta 0.00(\mathrm{~s}, 3 \mathrm{H}), 0.01(\mathrm{~s}, 3 \mathrm{H}), 0.86(\mathrm{~d}, J=$ $6.8 \mathrm{~Hz}, 3 \mathrm{H}), 0.86(\mathrm{~s}, 9 \mathrm{H}), 1.05(\mathrm{~s}, 3 \mathrm{H}), 1.06(\mathrm{~s}, 3 \mathrm{H}), 1.09-$ $1.15(\mathrm{~m}, 1 \mathrm{H}), 1.61-1.68(\mathrm{~m}, 1 \mathrm{H}), 1.76(\mathrm{td}, J=6.8,3.0 \mathrm{~Hz}$, $1 \mathrm{H}), 2.80(\mathrm{dd}, J=18.0,5.4 \mathrm{~Hz}, 1 \mathrm{H}), 2.91(\mathrm{dd}, J=18.0,7.4$ $\mathrm{Hz}, 1 \mathrm{H}), 2.94-2.98(\mathrm{~m}, 1 \mathrm{H}), 3.40(\mathrm{~s}, 3 \mathrm{H}), 3.44-3.56(\mathrm{~m}$, 3H), $3.79(\mathrm{~s}, 3 \mathrm{H}), 4.00-4.07(\mathrm{~m}, 1 \mathrm{H}), 4.66(\mathrm{~s}, 1 \mathrm{H}), 5.05(\mathrm{~s}, 1 \mathrm{H}), 5.44(\mathrm{td}, J=7.4,5.4,3.0 \mathrm{~Hz}$, $1 \mathrm{H}), 6.85(\mathrm{~d}, J=8.8 \mathrm{~Hz}, 2 \mathrm{H}), 7.29-7.46(\mathrm{~m}, 7 \mathrm{H}) .{ }^{13} \mathrm{C} \mathrm{NMR}\left(101 \mathrm{MHz}, \mathrm{CDCl}_{3}\right) \delta-5.6(\mathrm{q}),-5.6$ (q), $9.4(\mathrm{q}), 18.2(\mathrm{~s}), 21.3(\mathrm{q}),, 25.8(\mathrm{q}), 28.8(\mathrm{t}), 39.5(\mathrm{t}), 40.6(\mathrm{~d}), 49.5(\mathrm{~s}), 55.3(\mathrm{q}), 57.3(\mathrm{q})$, 66.7 (t), 70.0 (t), 70.9 (d), 77.0 (d), 82.2 (d), 100.6 (d), 113.4 (d), 127.1 (d), 127.4 (d), 128.7 (d), 128.8 (d), 131.4 (s), 136.9 (s), 159.7 (s), 169.7 (s), 211.3 (s). HRMS (+ESI): m/z calculated for $\mathrm{C}_{35} \mathrm{H}_{52} \mathrm{NaO}_{8} \mathrm{Si}[\mathrm{M}+\mathrm{Na}]^{+}$651.3324, found 651.3325.

(2R,3R)-7-((tert-Butyldimethylsilyl)oxy)-2-((4S)-2-(4-methoxyphenyl)-1,3-dioxan-4-yl)-6,6dimethyl-5-oxoheptan-3-yl (2S)-2-methoxy-2-phenylacetate (S3)

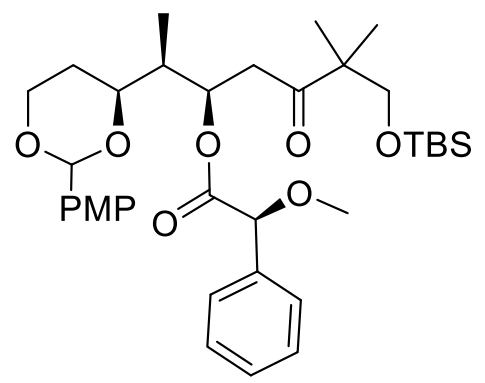

Aldol 10 (20 mg, $0.04 \mathrm{mmol})$ and (S)-MPA (35 mg, 0.21 mmol) afforded MPA derivative $\mathbf{S 3}(13 \mathrm{mg}, 52 \%)$. ${ }^{1} \mathrm{H}$ NMR $\left(400 \mathrm{MHz}, \mathrm{CDCl}_{3}\right) \delta-0.03(\mathrm{~s}, 3 \mathrm{H}),-0.03(\mathrm{~s}, 3 \mathrm{H}), 0.83(\mathrm{~s}, 9 \mathrm{H})$, $0.92(\mathrm{~s}, 6 \mathrm{H}), 0.99$ (d, $J=6.9 \mathrm{~Hz}, 3 \mathrm{H}), 1.37-1.43(\mathrm{~m}, 1 \mathrm{H})$, $1.70-1.87(\mathrm{~m}, 2 \mathrm{H}), 2.67(\mathrm{dd}, J=18.2,5.2 \mathrm{~Hz}, 1 \mathrm{H}), 2.81(\mathrm{dd}$, $J=18.2,7.5 \mathrm{~Hz}, 1 \mathrm{H}), 3.32(\mathrm{~s}, 3 \mathrm{H}), 3.42(\mathrm{~s}, 2 \mathrm{H}), 3.50-3.56$ $(\mathrm{m}, 1 \mathrm{H}), 3.72-3.77(\mathrm{~m}, 1 \mathrm{H}), 3.79(\mathrm{~s}, 3 \mathrm{H}), 4.13-4.19(\mathrm{~m}$, 1H), $4.67(\mathrm{~s}, 1 \mathrm{H}), 5.31(\mathrm{~s}, 1 \mathrm{H}), 5.48-5.53(\mathrm{~m}, 1 \mathrm{H}), 6.87(\mathrm{~d}, J=8.8 \mathrm{~Hz}, 2 \mathrm{H}), 7.29-7.43(\mathrm{~m}$, 7H). ${ }^{13} \mathrm{C}$ NMR (101 MHz, $\left.\mathrm{CDCl}_{3}\right) \delta-5.7$ (q), 9.4 (q), 18.1 (s), 21.1 (q), 21.2 (q), 25.8 (q), 29.0 (t), $39.4(\mathrm{t}), 40.9$ (d), 49.4 (s), $55.3(\mathrm{q}), 57.2(\mathrm{q}), 66.9$ (t), 69.8 (t), 71.3 (d), 77.4 (d), 82.8 (d), 100.8 (d), 113.5 (d), 127.0 (d), 127.2 (d), 128.5 (d), 128.5 (d), 131.5 (s), 136.4 (s), 159.8 (s), 169.9 (s), 211.0 (s). HRMS (+ESI): $m / z$ calculated for $\mathrm{C}_{35} \mathrm{H}_{52} \mathrm{NaO}_{8} \mathrm{Si}[\mathrm{M}+\mathrm{Na}]^{+} 651.3324$, found 651.3312 .

(3S,5R,6S)-1-((tert-Butyldimethylsilyl)oxy)-6-((4S)-2-(4-methoxyphenyl)-1,3-dioxan-4-yl)2,2-dimethylheptane-3,5-diol (S4) 


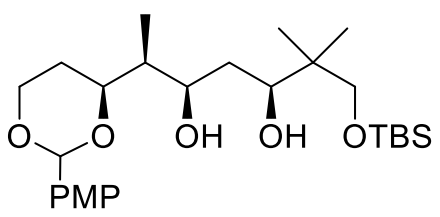

A solution of DIBAL $1 \mathrm{M}$ in THF $(7 \mathrm{~mL}, 7 \mathrm{mmol})$ was added during 10 minutes to a solution of aldol $10(1.33 \mathrm{~g}, 2.79 \mathrm{mmol})$ in THF ( $40 \mathrm{~mL})$ at $-100^{\circ} \mathrm{C}$ and the reaction was stirred $90 \mathrm{~min}$ at

$-78^{\circ} \mathrm{C}$. After this time, a saturated solution of Rochelle's salt (30

$\mathrm{mL}$ ) was added and the solution was stirred for $1 \mathrm{~h}$. The residue was extracted with $\mathrm{CH}_{2} \mathrm{Cl}_{2}$, the organic layer was dried over $\mathrm{Na}_{2} \mathrm{SO}_{4}$, filtered and the solvent was removed under reduced pressure. Purification by silica gel column chromatography with hexane-EtOAc (75:25) yielded syn-diol S4 (957 mg, 73\%). $[\alpha]_{\mathrm{D}}=-12.8\left(\mathrm{c} 1.0, \mathrm{CH}_{2} \mathrm{Cl}_{2}\right)$. IR (KBr film) v 3445 (br), 2956, 2930, 2857, 1615, 1250, 1101, 836, $777 \mathrm{~cm}^{-1} .{ }^{1} \mathrm{H}$ NMR (400 MHz, $\left.\mathrm{CDCl}_{3}\right) \delta 0.07$ (s, 6H), 0.84 (s, 3H), 0.89 (s, 3H), 0.90 (s, 9H), 1.06 (d, $J=7.0 \mathrm{~Hz}, 3 \mathrm{H}), 1.50-1.69$ (m, 4H), $1.87-1.99$ (m, 1H), $3.45-3.54(\mathrm{~m}, 2 \mathrm{H}), 3.72(\mathrm{~d}, J=10.6 \mathrm{~Hz}, 1 \mathrm{H}), 3.79(\mathrm{~s}, 3 \mathrm{H}), 3.92-4.02(\mathrm{~m}, 2 \mathrm{H}), 4.07$ (dt, $J=9.4,2.7 \mathrm{~Hz}, 1 \mathrm{H}$ ), 4.19 (brs, 1H), $4.23-4.29$ (m, 1H), 4.37 (brs, 1H), 5.49 (s, 1H), 6.87 (d, $J$ $=8.8 \mathrm{~Hz}, 2 \mathrm{H}), 7.40(\mathrm{~d}, J=8.7 \mathrm{~Hz}, 2 \mathrm{H}) .{ }^{13} \mathrm{C} \mathrm{NMR}\left(101 \mathrm{MHz}, \mathrm{CDCl}_{3}\right) \delta-5.7(\mathrm{q}),-5.7(\mathrm{q}), 8.9$ (q), $18.1(\mathrm{~s}), 19.2(\mathrm{q}), 22.2(\mathrm{q}), 25.8(\mathrm{q}), 28.9(\mathrm{t}), 35.0(\mathrm{t}), 38.2,43.1(\mathrm{~d}), 55.3(\mathrm{q}), 67.2(\mathrm{t}), 73.2$ (t), 73.4 (d), 79.3 (d), 80.9 (d), 100.9 (d), 113.5 (d), 127.2 (d), 131.5 , 159.7 . HRMS (ESI+): $\mathrm{m} / z$ calculated for $\mathrm{C}_{26} \mathrm{H}_{47} \mathrm{O}_{6} \mathrm{Si}[\mathrm{M}+\mathrm{H}]^{+}$483.3136, found 483.3126 .

(4S,6R)-4-[1-(tert-Butyldimethylsilyloxy)-2-methylpropan-2-yl]-6-[(1R)-1-((4S)-2-(4methoxyphenyl)-1,3-dioxan-4-yl)ethyl]-2,2-dimethyl-1,3-dioxane (11)

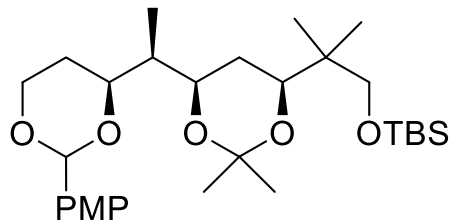

2-Methoxypropene $(0.015 \mathrm{~mL}, 0.16 \mathrm{mmol})$ was added to a cooled $\left(0^{\circ} \mathrm{C}\right)$ solution of $\mathbf{S} 4(30 \mathrm{mg}, 0.062 \mathrm{mmol})$ and PPTS $(1.5$ $\mathrm{mg}, 0.006 \mathrm{mmol})$ in $\mathrm{CH}_{2} \mathrm{Cl}_{2}(0.5 \mathrm{~mL})$. The reaction was stirred 30 minutes at. After this time, a $\mathrm{pH}=7$ buffer was added and the residue was extracted with $\mathrm{CH}_{2} \mathrm{Cl}_{2}$, the organic layer was dried over $\mathrm{Na}_{2} \mathrm{SO}_{4}$, filtered and the solvent was removed under reduced pressure. Purification by silica gel column chromatography with hexane-EtOAc $(95: 5)$ yielded $11(32 \mathrm{mg}, 98 \%)$ as a colorless oil. $[\alpha]_{D}=+6.9$ (c 1.0, $\mathrm{CH}_{2} \mathrm{Cl}_{2}$ ). IR ( $\mathrm{KBr}$ film) v 2955, 2861, 1737, 1377, 1249, $1101 \mathrm{~cm}^{-1} .{ }^{1} \mathrm{H}-\mathrm{RMN}(400 \mathrm{MHz}$, $\left.\mathrm{CDCl}_{3}\right): \delta 0.07(\mathrm{~s}, 3 \mathrm{H}), 0.81(\mathrm{~s}, 3 \mathrm{H}), 0.82(\mathrm{~s}, 3 \mathrm{H}), 0.89$ (s, 9H), $1.05(\mathrm{~d}, J=6.9 \mathrm{~Hz}, 3 \mathrm{H}), 1.26-$ $1.29(\mathrm{~m}, 3 \mathrm{H}), 1.33(\mathrm{~s}, 3 \mathrm{H}), 1.37(\mathrm{~s}, 3 \mathrm{H}), 1.42-1.50(\mathrm{~m}, 2 \mathrm{H}), 1.56-1.60(\mathrm{~m}, 1 \mathrm{H}), 1.93(\mathrm{qd}, J=$ 12.4, $5.0 \mathrm{~Hz}, 1 \mathrm{H}), 3.20(\mathrm{~d}, J=9.2 \mathrm{~Hz}, 1 \mathrm{H}), 3.44(\mathrm{~d}, J=9.2 \mathrm{~Hz}, 1 \mathrm{H}), 3.73(\mathrm{~d}, J=2.3 \mathrm{~Hz}, 1 \mathrm{H})$, $3.75(\mathrm{~d}, J=2.3 \mathrm{~Hz}, 1 \mathrm{H}), 3.80(\mathrm{~s}, 3 \mathrm{H}), 3.86-3.98(\mathrm{~m}, 3 \mathrm{H}), 4.27(\mathrm{dd}, J=11.3,3.9 \mathrm{~Hz}, 1 \mathrm{H}), 5.47$ (s, 1H), $6.89(\mathrm{~d}, J=8.8 \mathrm{~Hz}, 2 \mathrm{H}), 7.41(\mathrm{~d}, J=8.8 \mathrm{~Hz}, 2 \mathrm{H}) .{ }^{13} \mathrm{C}-\mathrm{RMN}\left(100.6 \mathrm{MHz}, \mathrm{CDCl}_{3}\right): \delta$ 5.4 (q) 10.3 (q) 18.4 (s), 19.5 (q), 19.9 (q), 20.8 (q), 25.0 (q), 28.2 (t), 28.8 (t), 30.4 (q), 36.7 (s), 43.1 (d), 55.4 (q), 67.4 (t), 68.8 (t), 72.0 (d), 78.0 (d), 98.2 (s), 101.1 (d), 113.6 (d), 127.4 (d), 131.8 (d), 159.9 (s), 190.9 (s). HRMS (+ESI): $\mathrm{m} / z$ calculated for $\mathrm{C}_{29} \mathrm{H}_{50} \mathrm{NaO}_{6} \mathrm{Si}[\mathrm{M}+\mathrm{Na}]^{+}$ 545.3269 , found 545.3253 . 
2-((4S,6R)-6-((1R)-1-((4S)-2-(4-Methoxyphenyl)-1,3-dioxan-4-yl)ethyl)-2,2-dimethyl-1,3dioxan-4-yl)-2-methylpropan-1-ol (S5)

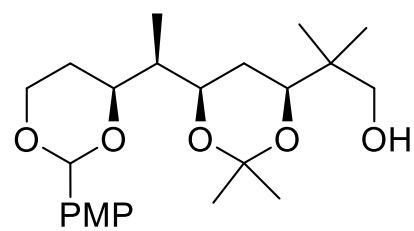

A solution of TBAF $1 \mathrm{M}$ in THF ( $5 \mathrm{~mL}, 4,9 \mathrm{mmol})$ was added to a solution of 11 (650 mg, $1.2 \mathrm{mmol})$ in dry THF $(60 \mathrm{~mL})$ at $\mathrm{rt}$ under $\mathrm{N}_{2}$ atmosphere and the reaction was stirred for $16 \mathrm{~h}$. After this time, the reaction was quenched with $\mathrm{NaCl}$ saturated solution (40 $\mathrm{mL}$ ) and the mixture was extracted with AcOEt. The organic layer was dried over $\mathrm{MgSO} 4$, filtered and evaporated under reduced pressure. Purification by silica gel column chromatography with hexane-EtOAc $(8: 2)$ yielded $\mathbf{S 5}(215 \mathrm{mg}, 44 \%)$ as a colorless oil. $[\alpha]_{D}=$ $+15,3\left(\mathrm{c} 1,0, \mathrm{CH}_{2} \mathrm{Cl}_{2}\right)$. IR (KBr film) v 3491, 2963, 1737, 1613, 1517, 1378, $1249 \mathrm{~cm}^{-1} .{ }^{1} \mathrm{H}-$ RMN (400 MHz, CDCl $\left.)_{3}\right): \delta$ 0,87 (s, 3H), 0,91 (s, 3H), 1,05 (d, $\left.J=6.9 \mathrm{~Hz}, 3 \mathrm{H}\right), 1,37(\mathrm{~s}, 3 \mathrm{H})$, 1,42 (s, 3H), 1,52 (ddd, $J=17.7,8.9,2.6 \mathrm{~Hz}, 2 \mathrm{H}), 1,62-1,69$ (m, 2H), 1,84-1,94 (m, 1H), 3,37 (d, $J=10.9 \mathrm{~Hz}, 1 \mathrm{H}), 3,54(\mathrm{~d}, J=10.9 \mathrm{~Hz}, 1 \mathrm{H}), 3,75$ (dd, $J=11.7,2.6 \mathrm{~Hz}, 1 \mathrm{H}), 3,80(\mathrm{~s}, 3 \mathrm{H})$, 3,85-3,95 (m, 1H), 3,93-3,99 (m, 2H), 4,24-4,30 (m, 1H), 5,46 (s, 1H), 6,88 (d, J = 8.7 Hz, 2H), $7,40(\mathrm{~d}, J=8.7 \mathrm{~Hz}, 2 \mathrm{H}) .{ }^{13} \mathrm{C}-\mathrm{RMN}\left(100.6 \mathrm{MHz}, \mathrm{CDCl}_{3}\right): \delta 10.2$ (q), 19.6 (q), 19.8 (q), 22.4 (q), $28.6(\mathrm{q}), 30.4(\mathrm{t}), 37.8(\mathrm{~s}), 42.9(\mathrm{~d}), 55.5(\mathrm{q}), 67.4(\mathrm{t}), 69.4(\mathrm{t}), 71.9(\mathrm{t}), 77.9(\mathrm{~d}), 78.0(\mathrm{~d}), 98.7$ (s), 101.2 (d), 113.7 (d), 127.5 (d), 131.8 (s), 160.0 (s). HRMS (ESI+): $m / z$ calculated $\mathrm{C}_{23} \mathrm{H}_{37} \mathrm{O}_{6}$ $[\mathrm{M}+\mathrm{H}]^{+}$409.2585, found 409.2573.

\section{2-((4S,6R)-6-((1R)-1-((4S)-2-(4-Methoxyphenyl)-1,3-dioxan-4-yl)ethyl)-2,2-dimethyl-1,3-}

dioxan-4-yl)-2-methylpropanal (12)<smiles>CC(C1CCOC([18F])O1)[C@H]1CC(C(C)(C)C=O)OC(C)(C)O1</smiles>

$\mathrm{NaHCO}_{3}(16 \mathrm{mg}, 0.19 \mathrm{mmol})$ and DMP $(73 \mathrm{mg}, 0.17 \mathrm{mmol})$ were added to a solution of alcohol $\mathbf{S 5}(60 \mathrm{mg}, 0.14 \mathrm{mmol})$ in $\mathrm{CH}_{2} \mathrm{Cl}_{2}$ $(2 \mathrm{~mL})$ and the solution was stirred for 30 minutes. The reaction mixture was diluted with a saturated solution of $\mathrm{Na}_{2} \mathrm{~S}_{2} \mathrm{O}_{3}$ and a saturated solution of $\mathrm{NaHCO}_{3}$ and the residue was extracted with $\mathrm{CH}_{2} \mathrm{Cl}_{2}$. The organic extracts were dried over $\mathrm{Na}_{2} \mathrm{SO}_{4}$, filtered and concentrated under reduced pressure isolating 12 (56 mg, 99\%) as a colorless oil. $[\alpha]_{\mathrm{D}}=+6,3\left(\mathrm{c} 1,0, \mathrm{CH}_{2} \mathrm{Cl}_{2}\right)$. IR ( $\mathrm{KBr}$ film $) \vee 2968,2853,1726,1379$, $1249 \mathrm{~cm}^{-1} .{ }^{1} \mathrm{H}-\mathrm{RMN}\left(400 \mathrm{MHz}, \mathrm{CDCl}_{3}\right): \delta 1.02-1.05(\mathrm{~m}, 9 \mathrm{H}), 1.33(\mathrm{~s}, 3 \mathrm{H}), 1.36-1.46(\mathrm{~m}, 5 \mathrm{H})$, 1.60-1.72 (m, 2H), 1.90-2.00 (m, 1H), 3.79 (s, 3H), 3.82-4.08 (m, 4H), 4.27 (dd, $J=11.4,3.9$ $\mathrm{Hz}, 1 \mathrm{H}), 5.30(\mathrm{~s}, 1 \mathrm{H}), 6.89(\mathrm{~d}, J=8.8 \mathrm{~Hz}, 2 \mathrm{H}), 7.39$ (d, $J=8.8 \mathrm{~Hz}, 2 \mathrm{H}), 9.57$ (s, 1H). ${ }^{13} \mathrm{C}-\mathrm{RMN}$ (100,6 MHz, $\left.\mathrm{CDCl}_{3}\right): \delta 10.2(\mathrm{q}), 16.7$ (q), 18.9 (q), 19.7 (q), $28.3(\mathrm{t}), 28.5$ (t), $30.0(\mathrm{q}), 42.9$ (d), 49.1 (s), 55.4 (q), 67.3 (t), 69.1 (d), 73.6 (d), 77.9 (d), 98.6 (s), 101.2 (d), 113.7 (d), 127.4 (d), 131.7 (s), 159.9 (s), 206.5 (d). HRMS (ESI+): $m / z$ calculated para $\mathrm{C}_{23} \mathrm{H}_{35} \mathrm{O}_{6}[\mathrm{M}+\mathrm{H}]^{+}$407,2428, found 407,2424 . 
(3R)-2-((4S,6R)-6-((1R)-1-((4S)-2-(4-methoxyphenyl)-1,3-dioxan-4-yl)ethyl)-2,2-dimethyl1,3-dioxan-4-yl)-2-methylhex-5-yn-3-ol (13)<smiles>C#CCC(O)C(C)(C)C1CC([C@H](C)C2CCOC(P)P2)OC(C)(C)O1</smiles>

Pyridine $(120 \mu \mathrm{L}, 1,5 \mathrm{mmol})$ and propargyl bromide $(220 \mu \mathrm{L}$, $1,5 \mathrm{mmol})$ were added over a solution of Indium (170 mg, 1,5 $\mathrm{mmol})$ and $(1 R, 2 S)$-2-Amino-1,2-diphenilethanol (320 mg, 1,5 $\mathrm{mmol})$ in dry THF $(3 \mathrm{~mL})$ at $\mathrm{rt}$ and the mixture was stirred 30 minutes at the same temperature. The reaction was cooled down to $-78^{\circ} \mathrm{C}$ and a solution of the aldehyde 12 (30 mg, $0.074 \mathrm{mmol})$ in THF $(0.5 \mathrm{~mL})$ was added and the reaction was stirred $1 \mathrm{~h}$ at $-78^{\circ} \mathrm{C}$ and $1 \mathrm{~h}$ at $\mathrm{rt}$. The reaction was quenched by addition of saturated solution of $\mathrm{NH}_{4} \mathrm{Cl}(4$ $\mathrm{mL}$ ) and extracted three times with $\mathrm{Et}_{2} \mathrm{O}$. The organic phase was dried over $\mathrm{Na}_{2} \mathrm{SO}_{4}$, filtered and the solvent was removed under reduced pressure. Purification by silica gel column chromatography with hexane-EtOAc (8:2) yielded $13(17 \mathrm{mg}, 52 \%)$ as a colorless oil. ${ }^{1} \mathrm{H}-\mathrm{RMN}$ $\left(400 \mathrm{MHz}, \mathrm{CDCl}_{3}\right): \delta$ 0,78 (s, 3H), 0,92 (s, 3H), 1,04 (d, $\left.J=7,0 \mathrm{~Hz}, 3 \mathrm{H}\right), 1,25-1,27(\mathrm{~m}$, 1H),1,34-1,36 (m, 1H), 1,36 (s, 3H), 1,45 (s, 3H), 1,47-1,49 (m, 1H), 1,61-1,69 (m, 1H), 1,88$1,99$ (m, 1H), 2,05 (t, $J=2,6 \mathrm{~Hz}, 1 \mathrm{H}), 2,32(\mathrm{ddd}, J=16,6,9,5,2,6 \mathrm{~Hz}, 1 \mathrm{H}), 2,44(\mathrm{dt}, J=16,6$, $3,5 \mathrm{~Hz}, 1 \mathrm{H}), 3,70-3,74(\mathrm{~m}, 1 \mathrm{H}), 3,78-3,81(\mathrm{~m}, 4 \mathrm{H}), 3,85-3,93(\mathrm{~m}, 1 \mathrm{H}), 3,92-4,21(\mathrm{~m}, 2 \mathrm{H}), 4,27$ $(\mathrm{dd}, J=11,3,3,5 \mathrm{~Hz}, 1 \mathrm{H}), 5,46(\mathrm{~s}, 1 \mathrm{H}), 6,87-6,89(\mathrm{~m}, 2 \mathrm{H}), 7,37-7,42(\mathrm{~m}, 2 \mathrm{H}) .{ }^{13} \mathrm{C}-\mathrm{RMN}(100,6$ $\left.\mathrm{MHz}, \mathrm{CDCl}_{3}\right): \delta$ 10,2 (q), 15,3 (q), 19,9 (q), 20,8 (q), 22,5 (t), 28,2 (t), 28,6 (t), 30,2 (q), 40,4 (d), 42,9 (s), 55,4 (q), 67,3 (t), 69,3 (d), 69,8 (d), 77,1 (d), 77,2 (d), 78,0 (s), 82,8 (s), 98,6 (s), 101,2 (d), 113,7 (d), 127,4 (d), 131,7 (d), 159,9 (s). HRMS (ESI+): m/z calculated para $\mathrm{C}_{26} \mathrm{H}_{39} \mathrm{O}_{6}[\mathrm{M}+\mathrm{H}]^{+} 447,2741$, found 447,27455 .

\section{2-((4S,6R)-6-((1R)-1-((4S)-2-(4-Methoxyphenyl)-1,3-dioxan-4-yl)ethyl)-2,2-dimethyl-1,3-} dioxan-4-yl)-2-methylhex-5-yn-3-yl (2S)-2-methoxy-2-phenylacetate (S6)

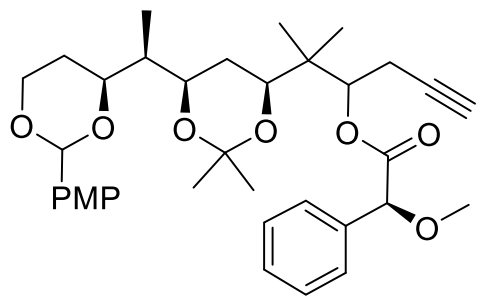

Following the previous general procedure: Aldol 13 (8 mg, $0.018 \mathrm{mmol})$ and $(S)$-MPA $(6 \mathrm{mg}, 0.032 \mathrm{mmol})$ afforded MPA derivative S6 (6 mg, 56\%). ${ }^{1} \mathrm{H}-\mathrm{RMN}(400 \mathrm{MHz}$, $\left.\mathrm{CDCl}_{3}\right): \delta 0,80(\mathrm{~s}, 3 \mathrm{H}), 0,83(\mathrm{~s}, 3 \mathrm{H}), 1,02(\mathrm{~d}, J=6,9 \mathrm{~Hz}, 3 \mathrm{H})$, 1,29 (s, 3H), 1,31 (s, 3H), 1,70-1,75 (m, 3H), 1,85-1,95 (m, $2 \mathrm{H}), 2,34-2,41(\mathrm{~m}, 1 \mathrm{H}), 2,48-2,53(\mathrm{~m}, 1 \mathrm{H}), 3,42(\mathrm{~s}, 1 \mathrm{H}), 3,47$ (s, 3H), 3,51-3,55 (m, 1H), 3,86-3,88 (m, 1H), 3,80 (s, 3H), 3,91-3,97 (m, 2H), 4,27 (dd, $J=$ $11,2,3,8 \mathrm{~Hz}, 1 \mathrm{H}), 4,78$ (s, 1H), 5,16 (dd, $J=9,5,3,8 \mathrm{~Hz}, 1 \mathrm{H}), 5,47$ (s, 1H), 6,88-6,90 (m, 2H), 7,29-7,33 (m, 3H), 7,40-7,45 (m, 4H). HRMS (ESI+): $\mathrm{m} / z$ calculated para $\mathrm{C}_{35} \mathrm{H}_{47} \mathrm{O}_{8}[\mathrm{M}+\mathrm{H}]^{+}$ 595,3265 , found 595,32724 . 
<smiles>C#CCC(OC(=O)c1ccccc1)C(C)(C)C1CC(C(C)C2CCOC([Pb])O2)OC(C)(C)O1</smiles>

Following the previous general procedure: Aldol $13(14 \mathrm{mg}$, $0.03 \mathrm{mmol}$ ) and $(R)$-MPA (40 mg, $0.24 \mathrm{mmol})$ afforded MPA derivative $\mathbf{S} 7(14 \mathrm{mg}, 78 \%)$. ${ }^{1} \mathrm{H}-\mathrm{RMN}\left(400 \mathrm{MHz}, \mathrm{CDCl}_{3}\right)$ : $\delta 0,62$ (sa, 6H), 0,99 (d, $J=6,9 \mathrm{~Hz}, 3 \mathrm{H}), 1,18$ (s, 3H), 1,23$1,24(\mathrm{~m}, 1 \mathrm{H}), 1,28(\mathrm{~s}, 3 \mathrm{H}), 1,38-1,41(\mathrm{~m}, 1 \mathrm{H}), 1,44-1,48(\mathrm{~m}$, $1 \mathrm{H}), 1,50-1,55(\mathrm{~m}, 1 \mathrm{H}), 1,83-1,84(\mathrm{~m}, 1 \mathrm{H}), 1,86-1,97(\mathrm{~m}, 1 \mathrm{H})$, 2,43-2,60 (m, 2H), 3,19-3,23 (m, 1H), 3,43 (s, 3H), 3,59-3,69 (m, 1H), 3,80 (s, 3H), 3,81-3,85 (m, 1H), 3,94 (dt, $J=12,2,2,6 \mathrm{~Hz}, 1 \mathrm{H}), 4,26$ (dd, $J=11,4,3,6 \mathrm{~Hz}, 1 \mathrm{H}), 4,78$ (s, 1H), 5,13 (dd, $J$ = 9,6, 3,6 Hz, 1H), 5,46 (s, 1H), 6,85-6,92 (m, 2H), 7,26-7,32 (m, 3H), 7,40-7,45 (m, 4H). HRMS (ESI+): $m / z$ calculated para $\mathrm{C}_{35} \mathrm{H}_{47} \mathrm{O}_{8}[\mathrm{M}+\mathrm{H}]^{+} 595,3265$, found 595,32711.

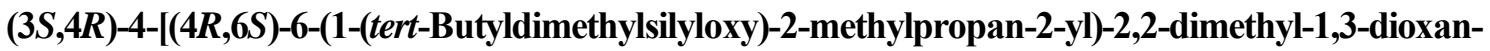
4-yl]-3-(4-methoxybenzyloxy)pentan-1-ol (14)<smiles>[B]OC(CCO)C(C)C1CC(C(C)(C)CCCCCC)OC(C)(C)O1</smiles>

A solution of DIBALH $1 \mathrm{M}$ in heptane $(9 \mathrm{~mL}, 9 \mathrm{mmol})$ was added to a solution of acetal $11(1.04 \mathrm{~g}, 1.98 \mathrm{mmol})$ in $\mathrm{CH}_{2} \mathrm{Cl}_{2}$ $(40 \mathrm{~mL})$ at $0{ }^{\circ} \mathrm{C}$. The reaction mixture was stirred at $40{ }^{\circ} \mathrm{C}$ for 16 $\mathrm{h}$ until TLC indicated complete conversion. Then a saturated solution of Rochelle's salt ( $30 \mathrm{~mL})$ was added and the mixture was stirred at $24 \mathrm{~h}$ until the formation of two layers. The organic solution was dried over $\mathrm{Na}_{2} \mathrm{SO}_{4}$, filtered and the solvent was removed under reduced pressure. Purification by silica gel column chromatography with hexane-EtOAc (72:25) yielded alcohol $14(920 \mathrm{mg}, 95 \% \mathrm{brsm})$ as a colorless oil and $69 \mathrm{mg}$ of recovered starting material 11. $[\alpha]_{\mathrm{D}}=$ 6.0 (c 0.5, $\mathrm{CH}_{2} \mathrm{Cl}_{2}$ ). IR (KBr film) v 3501 (br), 2955, 2857, 1737, 1513, 1377, 1249, $1092 \mathrm{~cm}^{-1}$. ${ }^{1} \mathrm{H}-\mathrm{RMN}\left(400 \mathrm{MHz}, \mathrm{CDCl}_{3}\right.$ ): $\delta 0.02$ (s, 6H), 0.78 (s, 3H), 0.79 (s, 3H), 0.89 (s, 9H), 0.97 (d, $J=$ $7.1 \mathrm{~Hz}, 3 \mathrm{H}), 1.24-1.26(\mathrm{~m}, 1 \mathrm{H}), 1.31(\mathrm{~s}, 3 \mathrm{H}), 1.32-1.34(\mathrm{~m}, 1 \mathrm{H}), 1.37(\mathrm{~s}, 3 \mathrm{H}), 1.79-1.84(\mathrm{~m}$, $3 \mathrm{H}), 3.19(\mathrm{~d}, J=9.2 \mathrm{~Hz}, 1 \mathrm{H}), 3.43(\mathrm{~d}, J=9.2 \mathrm{~Hz}, 1 \mathrm{H}), 3.60(\mathrm{dt}, J=7.1,4.9 \mathrm{~Hz}, 1 \mathrm{H}), 3.71-3.74$ (m, 3H), $3.80(\mathrm{~s}, 3 \mathrm{H}), 3.88-3.91(\mathrm{~m}, 1 \mathrm{H}), 4.47(\mathrm{q}, J=11.1,2 \mathrm{H}), 6.87(\mathrm{~d}, J=8.6 \mathrm{~Hz}, 2 \mathrm{H}), 7.26$ $(\mathrm{d}, J=8.6 \mathrm{~Hz}, 2 \mathrm{H}) .{ }^{13} \mathrm{C}-\mathrm{RMN}\left(100.6 \mathrm{MHz}, \mathrm{CDCl}_{3}\right): \delta-5.4$ (q), 10.9 (q), 18.4 (q), 19.5 (q), 19.9 (s), $20.9(\mathrm{q}), 26.1(\mathrm{q}), 28.9(\mathrm{t}), 30.3(\mathrm{q}), 33.3(\mathrm{t}), 38.7(\mathrm{~s}), 40.7(\mathrm{~d}), 55.4(\mathrm{q}), 61.4(\mathrm{t}), 68.8(\mathrm{t})$, 69.4 (d), 71.4 (t), 71.9 (d), 80.7 (d), 98.2 (s), 114.0 (d), 129.5 (d), 130.7 (d), 159.4 (s). HRMS (+ESI): $\mathrm{m} / z$ calculated for $\mathrm{C}_{29} \mathrm{H}_{53} \mathrm{O}_{6} \mathrm{Si}[\mathrm{M}+\mathrm{H}]^{+}$525.3606, found 525.3617 .

(3S,4R)-4-[(4R,6S)-6-(1-(tert-Butyldimethylsilyloxy)-2-methylpropan-2-yl)-2,2-dimethyl-1,3dioxan-4-yl]-3-(4-methoxybenzyloxy)pentanal (4) 


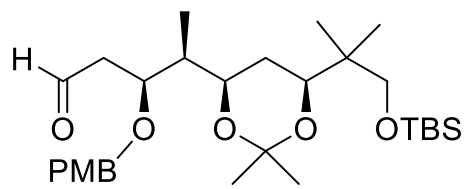

$\mathrm{NaHCO}_{3}(88 \mathrm{mg}, 1.05 \mathrm{mmol})$ and DMP (428 mg, $\left.0.98 \mathrm{mmol}\right)$ were added to a solution of alcohol $14(370 \mathrm{mg}, 0.7 \mathrm{mmol})$ in

$\mathrm{CH}_{2} \mathrm{Cl}_{2}(10 \mathrm{~mL})$ and the solution was stirred for 90 minutes.

The reaction mixture was diluted with a saturated solution of $\mathrm{Na}_{2} \mathrm{~S}_{2} \mathrm{O}_{3}$ and a saturated solution of $\mathrm{NaHCO}_{3}$ and the residue was extracted with $\mathrm{CH}_{2} \mathrm{Cl}_{2}$. The organic extracts were dried over $\mathrm{Na}_{2} \mathrm{SO}_{4}$, filtered and concentrated under reduced pressure. Purification by silica gel column chromatography with hexane-EtOAc (9:1) yielded aldehyde 4 (350 mg, 95\%) as a colorless oil. $[\alpha]_{\mathrm{D}}=-6.0\left(\mathrm{c} 1.0, \mathrm{CH}_{2} \mathrm{Cl}_{2}\right)$. IR (KBr film) $\vee 2957,2859,1725,1513,1249,1090 \mathrm{~cm}^{-1} .{ }^{1} \mathrm{H}-\mathrm{RMN}$ $\left(400 \mathrm{MHz}, \mathrm{CDCl}_{3}\right): \delta 0.02$ (s, 6H), 0.78 (s, 3H), 0.79 (s, 3H), 0.89 (s, 9H), 0.96 (d, J = $7.0 \mathrm{~Hz}$, $3 \mathrm{H}), 1.16-1.20(\mathrm{~m}, 1 \mathrm{H}), 1.28(\mathrm{~s}, 3 \mathrm{H}), 1.33-1.34(\mathrm{~m}, 1 \mathrm{H}), 1.35$ (s, 3H), 1.71-1.80 (m, 1H), 2.59$2.74(\mathrm{~m}, 2 \mathrm{H}), 3.17(\mathrm{~d}, J=9.1 \mathrm{~Hz}, 1 \mathrm{H}), 3.41(\mathrm{~d}, J=9.1 \mathrm{~Hz}, 1 \mathrm{H}), 3.71(\mathrm{dd}, J=11.7,2.3 \mathrm{~Hz}, 1 \mathrm{H})$, $3.78(\mathrm{~s}, 3 \mathrm{H}), 3.93-3.95(\mathrm{~m}, 1 \mathrm{H}), 3.96-3.98(\mathrm{~m}, 1 \mathrm{H}), 4.44(\mathrm{~s}, 2 \mathrm{H}), 6.87(\mathrm{~d}, J=8.7 \mathrm{~Hz}, 2 \mathrm{H}), 7.23$ $(\mathrm{d}, J=8.7 \mathrm{~Hz}, 2 \mathrm{H}), 9.46$ (t, $J=6.3 \mathrm{~Hz}, 1 \mathrm{H}) .{ }^{13} \mathrm{C}-\mathrm{RMN}\left(100.6 \mathrm{MHz}, \mathrm{CDCl}_{3}\right): \delta-5.4(\mathrm{q}), 10.6$ (q), 18.4 (s), 19.4 (q), 19.9 (q), 20.9 (q), 26.0 (q), 28.6 (t), 30.3 (q), 38.7 (s), 41.2 (d), 46.1 (t), 55.4 (q), 68.7 (t), 68.8 (d), $71.4(\mathrm{t}), 71.9$ (d), 76.2 (d), 98.2 (s), 114.1 (d), 129.4 (d), 130.6 (d), 159.4 (s), 202.5 (s). HRMS (ESI+): $m / z$ calculated for $\mathrm{C}_{29} \mathrm{H}_{51} \mathrm{O}_{6} \mathrm{Si}[\mathrm{M}+\mathrm{H}]^{+} 522.3407$, found 522.3412 .

\section{(E)-((4-Bromo-3-methoxybuta-1,3-dien-2-yl)oxy)trimethylsilane (15)}

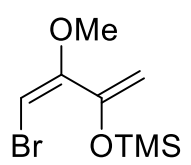
$\mathrm{Et}_{3} \mathrm{~N}(0.062 \mathrm{~mL}, 0.45 \mathrm{mmol})$ and TMSOTf $(0.065 \mathrm{~mL}, 0.36 \mathrm{mmol})$ were sequentially added to a solution of bromoketone $\mathbf{3}^{3}$ (55 $\mathrm{mg}, 0.3 \mathrm{mmol}$ ) in $\mathrm{CH}_{2} \mathrm{Cl}_{2}$ $(5 \mathrm{~mL})$ at $0{ }^{\circ} \mathrm{C}$. The reaction was stirred for $3 \mathrm{~h}$ at $\mathrm{rt}$ and quenched with diluted $\mathrm{NH}_{4} \mathrm{Cl}(5 \mathrm{~mL})$. The organic layer was washed three times with diluted $\mathrm{NH}_{4} \mathrm{Cl}$, dried over $\mathrm{MgSO}_{4}$ and concentrated under reduced pressure to obtain the silylenolether $\mathbf{1 5}$ as a brownish oil $(65 \mathrm{mg}, 86 \%)$. The crude was pure enough to continue the synthesis. ${ }^{1} \mathrm{H}$ NMR (400 MHz, $\left.\mathrm{CDCl}_{3}\right) \delta 0.24(\mathrm{~s}, 9 \mathrm{H}), 3.60(\mathrm{~s}, 3 \mathrm{H}), 4.65(\mathrm{~d}, J=1.4 \mathrm{~Hz}, 1 \mathrm{H}), 4.75(\mathrm{~d}, J=1.4 \mathrm{~Hz}, 1 \mathrm{H}), 5.39(\mathrm{~s}$, $1 \mathrm{H})$.

(5S,7S,8R,E)-1-Bromo-8-((4R,6S)-6-(1-((tert-butyldimethylsilyl)oxy)-2-methylpropan-2-yl)-2,2dimethyl-1,3-dioxan-4-yl)-5-hydroxy-2-methoxy-7-((4-methoxybenzyl)oxy)non-1-en-3-one (16)

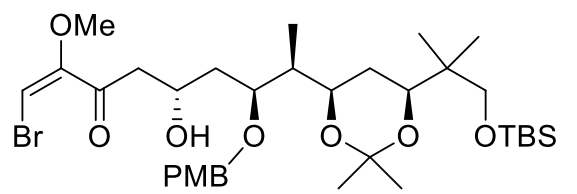

A solution of silylenolether 15 (35 mg, $0.122 \mathrm{mmol})$ in $\mathrm{CH}_{2} \mathrm{Cl}_{2}(0.5 \mathrm{~mL})$ was added to a cooled $\left(-78^{\circ} \mathrm{C}\right)$ solution of aldehyde 4 (46 mg, $0.077 \mathrm{mmol}$ ) in $\mathrm{CH}_{2} \mathrm{Cl}_{2}$ and then $\mathrm{BF}_{3} \cdot \mathrm{OEt}_{2}(12 \mu \mathrm{L}, 0.092 \mathrm{mmol})$ was added dropwise. The reaction was stirred at $-78^{\circ} \mathrm{C}$ for 90 minutes and quenched with saturated solution of $\mathrm{NaHCO}_{3}$ $(2 \mathrm{~mL})$ andwas extracted three times with $\mathrm{CH}_{2} \mathrm{Cl}_{2}$. The organic solution was dried over $\mathrm{Na}_{2} \mathrm{SO}_{4}$, filtrated and concentrated under reduced pressure. Purification by silica gel column cromatography with hexane-EtOAc (8:2) afforded $16(33.3 \mathrm{mg}, 62 \%)$ as a single diastereomer 
colorless oil. $[\alpha]_{\mathrm{D}}=-1.24\left(\mathrm{c} 0.5, \mathrm{CH}_{2} \mathrm{Cl}_{2}\right) . \mathrm{IR}(\mathrm{KBr}$ film $) \vee 3485,2955,2857,1708,1511,1378$, 1254, $1095 \mathrm{~cm}^{-1} .{ }^{1} \mathrm{H}$ NMR (400 MHz, $\left.\mathrm{CDCl}_{3}\right) \delta 0.01(\mathrm{~s}, 6 \mathrm{H}), 0.78(\mathrm{~s}, 3 \mathrm{H}), 0.79(\mathrm{~s}, 3 \mathrm{H}), 0.89$ (s, 9H), 0.97 (d, $J=7.0 \mathrm{~Hz}, 3 \mathrm{H}), 1.21-1.39(\mathrm{~m}, 3 \mathrm{H}), 1.30(\mathrm{~s}, 3 \mathrm{H}), 1.36(\mathrm{~s}, 3 \mathrm{H}), 1.62-1.82(\mathrm{~m}$, $3 \mathrm{H}), 2.81-2.85(\mathrm{~m}, 2 \mathrm{H}), 3.19(\mathrm{~d}, J=9.2 \mathrm{~Hz}, 1 \mathrm{H}), 3.43(\mathrm{~d}, J=9.2 \mathrm{~Hz}, 1 \mathrm{H}), 3.64(\mathrm{~s}, 3 \mathrm{H}), 3.69-$ $3.74(\mathrm{~m}, 2 \mathrm{H}), 3.79(\mathrm{~s}, 3 \mathrm{H}), 3.87-3.93(\mathrm{~m}, 1 \mathrm{H}), 4.28-4.52(\mathrm{~m}, 2 \mathrm{H}), 4.49(\mathrm{~s}, 1 \mathrm{H}), 5.66(\mathrm{~s}, 1 \mathrm{H})$, $6.87(\mathrm{~d}, J=8.7 \mathrm{~Hz}, 2 \mathrm{H}), 7.26(\mathrm{~d}, J=8.7 \mathrm{~Hz}, 2 \mathrm{H}) .{ }^{13} \mathrm{C}-\mathrm{RMN}\left(100.6 \mathrm{MHz}, \mathrm{CDCl}_{3}\right)$ : $-5.6(\mathrm{q}),-5.5$ (q), 10.5 (q), $18.2(\mathrm{~s}), 19.3(\mathrm{q}), 19.8(\mathrm{q}), 20.7$ (q), 25.9 (q), 28.9 (t), 30.2 (q), 37.8 (t), 38.5 (s), $41.1(\mathrm{~d}), 47.1(\mathrm{t}), 55.2(\mathrm{q}), 56.0(\mathrm{q}), 64.9(\mathrm{~d}), 68.7(\mathrm{t}), 69.2(\mathrm{~d}), 71.8(\mathrm{~d}), 71.8(\mathrm{t}), 78.3(\mathrm{~d}), 84.2$ (d), 98.0 (s), 113.8 (d), 129.4 (d), 130.8 (s), 152.9 (s), 159.1 (s), 197.6 (s). HRMS (ESI+): m/z calculated for $\mathrm{C}_{34} \mathrm{H}_{57} \mathrm{BrNaO}_{8} \mathrm{Si}[\mathrm{M}+\mathrm{Na}]^{+}$723.2898, found 723.2894.

\section{(E)-4-Bromo-1-((2S,4S,6S)-6-((R)-1-((4R,6S)-6-(1-((tert-butyldimethylsilyl)oxy)-2-}

methylpropan-2-yl)-2,2-dimethyl-1,3-dioxan-4-yl)ethyl)-2-(4-methoxyphenyl)-1,3-dioxan-4-yl)3-methoxybut-3-en-2-one (S8)

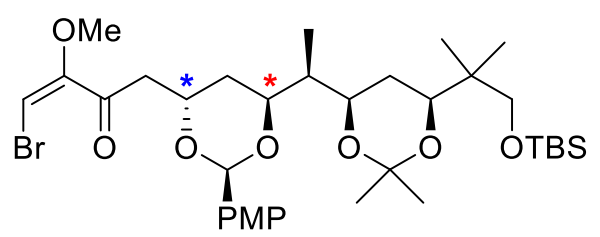

DDQ (15 mg, $0.066 \mathrm{mmol}$ ) was added to a solution of aldol $16(30 \mathrm{mg}, 0.042 \mathrm{mmol})$ in dry $\mathrm{CH}_{2} \mathrm{Cl}_{2}(3 \mathrm{~mL})$ and the reaction was stirred for 30 minutes. After this time, the reaction was filtered through a pad of celite and a saturated solution of $\mathrm{NaHCO}_{3}$ was added to the resulting solution. The solution was washed three times with $\mathrm{NaHCO}_{3}$. The organic layer was dried over $\mathrm{Na}_{2} \mathrm{SO}_{4}$, filtered and the solvent was removed under reduced pressure. Purification by silica gel column chromatography with hexane-EtOAc (85:15) yielded S8 (28 mg, 98\%) as a colorless oil. ${ }^{1} \mathrm{H}$ NMR (400 MHz, $\left.\mathrm{CDCl}_{3}\right) \delta 0.01(\mathrm{~s}, 6 \mathrm{H}), 0.79-0.81(\mathrm{~m}, 6 \mathrm{H}), 0.88(\mathrm{~s}, 9 \mathrm{H}), 1.05(\mathrm{~d}, J=6.9 \mathrm{~Hz}, 3 \mathrm{H}), 1.22-1.27$ $(\mathrm{m}, 1 \mathrm{H}), 1.31(\mathrm{~s}, 3 \mathrm{H}), 1.35(\mathrm{~s}, 3 \mathrm{H}), 1.42-1.46(\mathrm{~m}, 1 \mathrm{H}), 1.51-1.55(\mathrm{~m}, 1 \mathrm{H}), 1.61(\mathrm{td}, J=6.9$, $3.9 \mathrm{~Hz}, 1 \mathrm{H}), 2.09-2.18(\mathrm{~m}, 1 \mathrm{H}), 3.17-3.19(\mathrm{~m}, 1 \mathrm{H}), 3.21-3.24(\mathrm{~m}, 1 \mathrm{H}), 3.35-3.41(\mathrm{~m}, 1 \mathrm{H})$, $3.42-3.45(\mathrm{~m}, 1 \mathrm{H}), 3.66(\mathrm{~s}, 3 \mathrm{H}), 3.74(\mathrm{dd}, J=11.7,2.2 \mathrm{~Hz}, 1 \mathrm{H}), 3.79(\mathrm{~s}, 3 \mathrm{H}), 3.91(\mathrm{dt}, J=$ 11.5, 3.9, $1.9 \mathrm{~Hz}, 1 \mathrm{H}), 4.01(\mathrm{dt}, J=8.8,3.9,2.3 \mathrm{~Hz}, 1 \mathrm{H}), 4.82(\mathrm{q}, J=6.8 \mathrm{~Hz}, 1 \mathrm{H}), 5.67(\mathrm{~s}, 1 \mathrm{H})$, $5.75(\mathrm{~s}, 1 \mathrm{H}), 6.86(\mathrm{~d}, J=8.8 \mathrm{~Hz}, 2 \mathrm{H}), 7.38(\mathrm{~d}, J=8.8 \mathrm{~Hz}, 2 \mathrm{H}) .{ }^{13} \mathrm{C}-\mathrm{RMN}\left(100.6 \mathrm{MHz}, \mathrm{CDCl}_{3}\right)$ : ${ }^{13} \mathrm{C}$ NMR (101 MHz, $\mathrm{CDCl}_{3}$ ) $\delta-5.6$ (q), -5.5 (q), 10.0 (q), 18.2 (s), 19.3 (q), 19.7 (q), 20.7 (q), $25.9(\mathrm{q}), 27.9(\mathrm{t}), 30.2(\mathrm{q}), 30.9(\mathrm{t}), 38.5(\mathrm{~s}), 41.4(\mathrm{t}), 42.7(\mathrm{~d}), 55.3(\mathrm{q}), 56.0(\mathrm{q}), 68.7(\mathrm{t}), 68.9$ (d), 69.0 (d), 71.9 (d), 73.2 (d), 84.2 (d), 94.8 (d), 98.1 (s), 113.5 (d), 127.2 (d), 131.5 (s), 153.0 (s), 159.7 (s), 195.1 (s). HRMS (ESI+): $m / z$ calculated for $\mathrm{C}_{34} \mathrm{H}_{56} \mathrm{BrO}_{8} \mathrm{Si}[\mathrm{M}+\mathrm{H}]^{+}$699.2922, found 699.2906 .

(5S,7S,8R,E)-1-Bromo-8-((4R,6S)-6-(1-((tert-butyldimethylsilyl)oxy)-2-methylpropan-2-yl)-2,2dimethyl-1,3-dioxan-4-yl)-2-methoxy-7-((4-methoxybenzyl)oxy)-5-((triethylsilyl)oxy)non-1-en3-one (S9) 


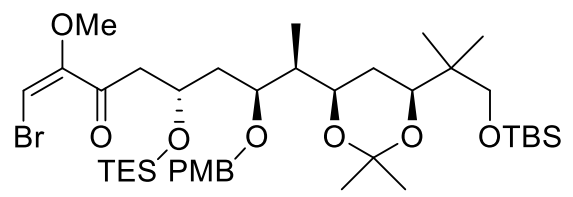

Imidazole ( $85 \mathrm{mg}, 1.24 \mathrm{mmol})$, TESCl $(0.16 \mathrm{~mL}, 0.93$ mmol) and DMAP (3 $\mathrm{mg}, 0.03 \mathrm{mmol})$ were added

sequentially to a solution of $\mathbf{1 6}(219 \mathrm{mg}, 0.31 \mathrm{mmol})$ in $\mathrm{CH}_{2} \mathrm{Cl}_{2}(6 \mathrm{~mL})$. The cloudy solution was stirred 90 minutes until TLC analysis showed complete conversion of the starting material. The reaction was quenched with saturated solution of $\mathrm{NH}_{4} \mathrm{Cl}(6 \mathrm{~mL})$ and extracted three times with $\mathrm{CH}_{2} \mathrm{Cl}_{2}$. The organic layers were dried over $\mathrm{MgSO}_{4}$, filtered and the solvent was evaporated under reduced pressure. Purification with deactivated $\left(1 \% \mathrm{Et}_{3} \mathrm{~N}\right)$ silica gel column cromatography with hexane-EtOAc (95:5) afforded S9 (235 mg, 96\%) as a colorless oil. $[\alpha]_{\mathrm{D}}=-1.46\left(\mathrm{c} 0.5, \mathrm{CH}_{2} \mathrm{Cl}_{2}\right)$. IR (KBr film) v 2955, 2877, 1705, 1517, 1384, 1251, $1083 \mathrm{~cm}^{-1} .{ }^{1} \mathrm{H}$ NMR $\left(400 \mathrm{MHz}, \mathrm{CDCl}_{3}\right) \delta$ 0.01 (s, 6H), 0.59 (2q, $J=7.9 \mathrm{~Hz}, 6 \mathrm{H}), 0.75$ (s, 3H), 0.76 (s, 3H), 0.89 (s, 9H), 0.94 (t, $J=7.9$ $\mathrm{Hz}, 9 \mathrm{H}), 0.95$ (d, J = 7.0 Hz, 3H), $1.23-1.27(\mathrm{~m}, 2 \mathrm{H}), 1.30$ (s, 3H), 1.35 (s, 3H), $1.70-1.76$ (m, 2H), $2.82(\mathrm{dd}, J=16.2,5.8 \mathrm{~Hz}, 1 \mathrm{H}), 2.94(\mathrm{dd}, J=16.2,6.4 \mathrm{~Hz}, 1 \mathrm{H}), 3.15(\mathrm{~d}, J=9.2 \mathrm{~Hz}$, $1 \mathrm{H}), 3.43(\mathrm{~d}, J=9.2 \mathrm{~Hz}, 1 \mathrm{H}), 3.53-3.57(\mathrm{~m}, 1 \mathrm{H}), 3.59(\mathrm{~s}, 3 \mathrm{H}), 3.66-3.71(\mathrm{~m}, 1 \mathrm{H}), 3.79$ (s, $3 \mathrm{H}), 3.81-3.86(\mathrm{~m}, 1 \mathrm{H}), 4.32-4.40(\mathrm{~m}, 1 \mathrm{H}), 4.38-4.47(\mathrm{~m}, 3 \mathrm{H}), 5.57(\mathrm{~s}, 1 \mathrm{H}), 6.85(\mathrm{~d}, J=$ $8.7 \mathrm{~Hz}, 2 \mathrm{H}), 7.24(\mathrm{~d}, J=8.6 \mathrm{~Hz}, 2 \mathrm{H}) .{ }^{13} \mathrm{C}-\mathrm{RMN}\left(100.6 \mathrm{MHz}, \mathrm{CDCl}_{3}\right) \delta-5.6$ (q), -5.5 (q), 5.1 (t), 7.0 (q), 10.5 (q), 18.3 (s), 19.1 (q), 19.8 (q), 20.8 (q), 25.9 (q), 29.0 (t), 30.2 (q), 38.5 (s), 39.9 (t), $40.8(\mathrm{~d}), 48.5(\mathrm{t}), 55.2(\mathrm{q}), 55.8(\mathrm{q}), 66.8(\mathrm{~d}), 68.7(\mathrm{t}), 69.5$ (d), $70.3(\mathrm{t}), 71.5(\mathrm{~d}), 77.7$ (d), 83.5 (d), 97.9 (s), 113.6 (d), 128.9 (d), 131.2 (s), 153.4 (s), 158.9 (s), 195.8 (s). HRMS (ESI+): $\mathrm{m} / z$ calculated for $\mathrm{C}_{40} \mathrm{H}_{75} \mathrm{BrNO}_{8} \mathrm{Si}_{2}\left[\mathrm{M}+\mathrm{NH}_{4}\right]^{+}$832.4209, found 832.4200.

\section{(((5R,7S,8R,E)-1-Bromo-8-((4R,6S)-6-(1-((tert-butyldimethylsilyl)oxy)-2-methylpropan-2-yl)-}

\section{2,2-dimethyl-1,3-dioxan-4-yl)-2-methoxy-7-((4-methoxybenzyl)oxy)-3-methylenenon-1-en-5-} yl)oxy)triethylsilane (S10)

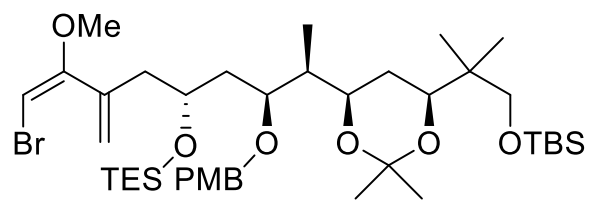

A solution of Tebbe reagent in toluene $(0.5 \mathrm{M}, 1.1 \mathrm{~mL}$, $0.55 \mathrm{mmol})$ was added to a solution of $\mathbf{S 9}(137 \mathrm{mg}$, $0.17 \mathrm{mmol})$ and pyridine $(0.05 \mathrm{~mL}, 0.55 \mathrm{mmol})$ in THF $(3.5 \mathrm{~mL})$ at $0{ }^{\circ} \mathrm{C}$. The reaction mixture was warmed to rt and then heated up at $50{ }^{\circ} \mathrm{C}$ during 2 hours. The reaction was quenched at $\mathrm{rt}$ with saturated solution of Rochelle's salt $(3 \mathrm{~mL})$ and extracted three times with $\mathrm{Et}_{2} \mathrm{O}$. The organic phase was dried over $\mathrm{MgSO}_{4}$, filtered and the solvent was evaporated under reduced pressure. Purification by silica gel column chromatography with hexane-EtOAc (95:5) yielded $\mathbf{S 1 0}$ (123 mg, 92\%) as a colorless oil. $[\alpha]_{\mathrm{D}}=-0.48\left(\mathrm{c} 0.5, \mathrm{CH}_{2} \mathrm{Cl}_{2}\right)$. IR $(\mathrm{KBr}$ film $) \vee 2955,2874,1613,1514,1373$, $1251,1092 \mathrm{~cm}^{-1} .{ }^{1} \mathrm{H}$ NMR (400 MHz, $\left.\mathrm{CDCl}_{3}\right) \delta{ }^{1} \mathrm{H}$ NMR (400 MHz, Chloroform- $d$ ) $\delta 0.01$ (s, $6 \mathrm{H}), 0.59(2 \mathrm{q}, J=7.9 \mathrm{~Hz}, 6 \mathrm{H}), 0.75(\mathrm{~s}, 6 \mathrm{H}), 0.88(\mathrm{~s}, 9 \mathrm{H}), 0.94(\mathrm{~d}, J=6.9 \mathrm{~Hz}, 3 \mathrm{H}), 0.96(\mathrm{t}, J=$ $7.9 \mathrm{~Hz}, 9 \mathrm{H}), 1.23-1.30(\mathrm{~m}, 2 \mathrm{H}), 1.30(\mathrm{~s}, 3 \mathrm{H}), 1.35$ (s, 3H), $1.49-1.55(\mathrm{~m}, 1 \mathrm{H}), 1.71-1.77$ (m, 1H), $1.81-1.90(\mathrm{~m}, 1 \mathrm{H}), 2.30(\mathrm{dd}, J=13.8,8.8 \mathrm{~Hz}, 1 \mathrm{H}), 2.60(\mathrm{dd}, J=13.8,4.2 \mathrm{~Hz}, 1 \mathrm{H})$, 
$3.15(\mathrm{~d}, J=9.2 \mathrm{~Hz}, 1 \mathrm{H}), 3.44(\mathrm{~d}, J=9.2 \mathrm{~Hz}, 1 \mathrm{H}), 3.51(\mathrm{~s}, 3 \mathrm{H}), 3.61-3.65(\mathrm{~m}, 1 \mathrm{H}), 3.68(\mathrm{dd}, J$ $=11.2,2.9 \mathrm{~Hz}, 1 \mathrm{H}), 3.80(\mathrm{~s}, 3 \mathrm{H}), 3.80-3.85(\mathrm{~m}, 1 \mathrm{H}), 3.94(\mathrm{dt}, J=8.8,5.2,4.2 \mathrm{~Hz}, 1 \mathrm{H}), 4.37-$ $4.48(\mathrm{~m}, 2 \mathrm{H}), 5.27(\mathrm{~s}, 1 \mathrm{H}), 5.34(\mathrm{~s}, 1 \mathrm{H}), 5.39(\mathrm{~s}, 1 \mathrm{H}), 6.85(\mathrm{~d}, J=8.6 \mathrm{~Hz}, 2 \mathrm{H}), 7.24(\mathrm{~d}, J=8.6$ $\mathrm{Hz}, 2 \mathrm{H}) .{ }^{13} \mathrm{C}-\mathrm{RMN}\left(100.6 \mathrm{MHz}, \mathrm{CDCl}_{3}\right) \delta-5.6(\mathrm{q}),-5.5$ (q), 5.3 (t), 7.0 (q), 10.6 (q), 18.3 (s), 19.0 (q), 19.8 (q), 20.9 (q), 25.9 (q), 29.1 (t), $30.3(\mathrm{q}), 38.5(\mathrm{~s}), 38.8(\mathrm{t}), 41.1$ (d), $43.4(\mathrm{t}), 55.2$ (q), $55.3(\mathrm{q}), 68.5(\mathrm{~d}), 68.7(\mathrm{t}), 69.8(\mathrm{~d}), 70.3(\mathrm{t}), 71.5$ (d), 77.6 (s), 78.3 (d), 97.9 (s), 113.5 (d), $121.4(\mathrm{t}), 128.6$ (d), 131.7 (s), 139.3 (s), 158.8 (s), 158.9 (s). HRMS (ESI+): $\mathrm{m} / z$ calculated for $\mathrm{C}_{41} \mathrm{H}_{74} \mathrm{BrO}_{7} \mathrm{Si}_{2}[\mathrm{M}+\mathrm{H}]^{+}$813.4151, found 813.4151.

(5R,7S,8R,E)-1-Bromo-8-((4R,6S)-6-(1-((tert-butyldimethylsilyl)oxy)-2-methylpropan-2-yl)-2,2dimethyl-1,3-dioxan-4-yl)-2-methoxy-7-((4-methoxybenzyl)oxy)-3-methylenenon-1-en-5-ol (17)

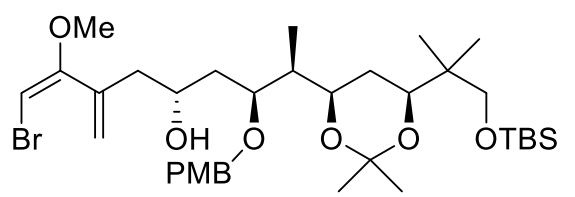
PPTS (10 $\mathrm{mg}, 0.03 \mathrm{mmol}$ ) was added to a solution of $\mathbf{S 1 0}$ $(123 \mathrm{mg}, 0.15 \mathrm{mmol})$ in $\mathrm{MeOH}(5 \mathrm{~mL})$ at $0^{\circ} \mathrm{C}$ and the reaction mixture was stirred 30 minutes at rt. After this time was quenched with saturated solution of $\mathrm{NaHCO}_{3}$

(5mL) and extracted three times with $\mathrm{CH}_{2} \mathrm{Cl}_{2}$. The organic phase was dried over $\mathrm{MgSO}_{4}$, filtered and the solvent was evaporated under reduced pressure. Purification by silica gel column chromatography with hexane-EtOAc (85:15) yielded 17 (107 mg, quant.) as a colorless oil. $[\alpha]_{\mathrm{D}}=-1.30\left(\mathrm{c} 0.5, \mathrm{CH}_{2} \mathrm{Cl}_{2}\right) . \mathrm{IR}(\mathrm{KBr}$ film $) \vee 3470(\mathrm{br}), 2955,2854,1607,1514,1248,1092$ $\mathrm{cm}^{-1} .{ }^{1} \mathrm{H}$ NMR (400 MHz, $\left.\mathrm{CDCl}_{3}\right) \delta 0.01(\mathrm{~s}, 6 \mathrm{H}), 0.78$ (s, 3H), 0.78 (s, 3H), 0.89 (s, 9H), 0.97 $(\mathrm{d}, J=7.0 \mathrm{~Hz}, 3 \mathrm{H}), 1.24-1.28(\mathrm{~m}, 2 \mathrm{H}), 1.31(\mathrm{~s}, 3 \mathrm{H}), 1.35(\mathrm{~s}, 3 \mathrm{H}), 1.61-1.71(\mathrm{~m}, 2 \mathrm{H}), 1.71-$ $1.77(\mathrm{~m}, 1 \mathrm{H}), 2.31(\mathrm{dd}, J=13.8,8.5 \mathrm{~Hz}, 1 \mathrm{H}), 2.44(\mathrm{dd}, J=13.8,4.1 \mathrm{~Hz}, 1 \mathrm{H}), 3.17(\mathrm{~d}, J=9.2$ $\mathrm{Hz}, 1 \mathrm{H}), 3.43$ (d, J = 9.2 Hz, 1H), 3.57 (s, 3H), $3.68-3.74$ (m, 2H), 3.80 (s, 3H), $3.83-3.91$ (m, 2H), 4.49 (s, 2H), $5.32(\mathrm{~s}, 1 \mathrm{H}), 5.38(\mathrm{~s}, 2 \mathrm{H}), 6.86(\mathrm{~d}, J=8.6 \mathrm{~Hz}, 2 \mathrm{H}), 7.26(\mathrm{~d}, J=8.6 \mathrm{~Hz}$, 2H). ${ }^{13} \mathrm{C}-\mathrm{RMN}\left(100.6 \mathrm{MHz}, \mathrm{CDCl}_{3}\right) \delta-5.6$ (q), -5.5 (q), 10.5 (q), 18.3 (s), 19.2 (q), 19.8 (q), $20.8(\mathrm{q}), 25.9(\mathrm{q}), 29.0(\mathrm{t}), 30.2(\mathrm{q}), 38.0(\mathrm{t}), 38.5(\mathrm{~s}), 41.3(\mathrm{~d}), 43.1(\mathrm{t}), 55.3(\mathrm{q}), 55.7(\mathrm{q}), 66.7$ (d), 68.7 (t), 69.3 (d), 71.7 (d), 71.8 (t), 78.4 (d), 78.6 (d), 98.0 (s), 113.8 (d), 122.0 (t), 129.4 (d), 131.0 (s), 139.7 (s), 159.1 (s), 159.1 (s). HRMS (ESI+): $\mathrm{m} / z$ calculated for $\mathrm{C}_{35} \mathrm{H}_{59} \mathrm{BrNaO}_{7} \mathrm{Si}$ $[\mathrm{M}+\mathrm{Na}]^{+}$721.3106, found 723.3090 .

(5S,7S,8R,E)-1-Bromo-8-((4R,6S)-6-(1-((tert-butyldimethylsilyl)oxy)-2-methylpropan-2-yl)-2,2dimethyl-1,3-dioxan-4-yl)-2-methoxy-7-((4-methoxybenzyl)oxy)-3-methylenenon-1-en-5-ol (1)<smiles>C=C(Br)/C(=C\O)CC(O)CC(O[18OH])C(C)C1CC(C(C)(C)CO[GaH])OC(C)(C)O1</smiles>

DIAD $(0.21 \mathrm{~mL}, 1.08 \mathrm{mmol})$ was slowly added to a solution of 17 (80 mg, $0.11 \mathrm{mmol}$ ), p-nitrobenzoic acid (160 mg, 0.96 $\mathrm{mmol})$ and $\mathrm{PPh}_{3}(282 \mathrm{mg}, 1.08 \mathrm{mmol})$ in benzene $(3 \mathrm{~mL})$ and the yellowish solution was stirred for $3 \mathrm{~h}$ at $\mathrm{rt}$. The reaction was quenched with saturated solution of $\mathrm{NaHCO}_{3}(3 \mathrm{~mL})$ and extracted three times with $\mathrm{Et}_{2} \mathrm{O}$. The organic layer was dried 
over $\mathrm{MgSO}_{4}$, filtered and evaporated under reduced pressure. The crude was filtered through a plug of silica using hexane-EtOAc 9:1 to eliminate the $p$-nitrobenzoic acid and $\mathrm{PPh}_{3} \mathrm{O}\left(\mathrm{R}_{\mathrm{f}}=\right.$ 0.1). After evaporation the resulting crude was dissolved in $\mathrm{MeOH}(5 \mathrm{~mL}), \mathrm{K}_{2} \mathrm{CO}_{3}(180 \mathrm{mg}$, $1.30 \mathrm{mmol}$ ) was added and the mixture was stirred for $2 \mathrm{~h}$ until TLC indicated total hydrolysis. The reaction was quenched with saturated solution of $\mathrm{NH}_{4} \mathrm{Cl}(5 \mathrm{~mL})$ and extracted three times with AcOEt. The organic layer was dried over $\mathrm{MgSO}_{4}$, filtered, evaporated under reduced pressure and purified by silica gel column chromatography with hexane-EtOAc 9:1 to obtain 1 as a colorless oil (47 mg, $58 \%$ for two steps). $[\alpha]_{\mathrm{D}}=+1.79\left(\mathrm{c} 1, \mathrm{CH}_{2} \mathrm{Cl}_{2}\right) . \mathrm{IR}(\mathrm{KBr}$ film $) \vee 3470$ (br), 2955, 2854, 1607, 1514, 1248, $\left.1092 \mathrm{~cm}^{-1} .{ }^{1} \mathrm{H} \mathrm{NMR} \mathrm{(400} \mathrm{MHz,} \mathrm{CDCl}_{3}\right) \delta 0.01$ (s, 6H), 0.76 (s, 3H), 0.78 (s, 3H), 0.89 (s, 9H), $0.94(\mathrm{~d}, J=7.0 \mathrm{~Hz}, 3 \mathrm{H}), 1.25-1.30(\mathrm{~m}, 2 \mathrm{H}), 1.32(\mathrm{~s}, 3 \mathrm{H})$, $1.37(\mathrm{~s}, 3 \mathrm{H}), 1.65-1.72(\mathrm{~m}, 1 \mathrm{H}), 1.76-1.84(\mathrm{~m}, 2 \mathrm{H}), 2.34-2.47(\mathrm{~m}, 2 \mathrm{H}), 3.17(\mathrm{~d}, J=9.2 \mathrm{~Hz}$, $1 \mathrm{H}), 3.43(\mathrm{~d}, J=9.2 \mathrm{~Hz}, 1 \mathrm{H}), 3.57(\mathrm{~s}, 3 \mathrm{H}), 3.62-3.66(\mathrm{~m}, 1 \mathrm{H}), 3.67-3.75(\mathrm{~m}, 2 \mathrm{H}), 3.79(\mathrm{~s}$, 3H), $3.84-3.91(\mathrm{~m}, 1 \mathrm{H}), 4.42(\mathrm{~d}, J=11.0 \mathrm{~Hz}, 1 \mathrm{H}), 4.49(\mathrm{~d}, J=11.0 \mathrm{~Hz}, 1 \mathrm{H}), 5.32(\mathrm{~s}, 1 \mathrm{H}), 5.37$ $(\mathrm{s}, 2 \mathrm{H}), 6.86(\mathrm{~d}, J=8.6 \mathrm{~Hz}, 2 \mathrm{H}), 7.24(\mathrm{~d}, J=8.6 \mathrm{~Hz}, 2 \mathrm{H}) .{ }^{13} \mathrm{C}-\mathrm{RMN}\left(100.6 \mathrm{MHz}, \mathrm{CDCl}_{3}\right) \delta-5.6$ (q), -5.5 (q), 10.8 (q), 18.2 (s), 19.2 (q), 19.8 (q), 20.8 (q), 25.9 (q), 29.0 (t), 30.2 (q), 37.1 (t), $38.5(\mathrm{~s}), 40.4(\mathrm{~d}), 42.8(\mathrm{t}), 55.2(\mathrm{q}), 55.6(\mathrm{q}), 68.7(\mathrm{t}), 69.1(\mathrm{~d}), 69.5(\mathrm{~d}), 70.8(\mathrm{t}), 71.6(\mathrm{~d}), 78.6$ (d), 80.8 (d), $98.0(\mathrm{~s}), 113.8$ (d), 121.9 (t), 129.3 (d), 130.5 (s), 139.5 (s), 159.0 (s), 159.2 (s). HRMS (ESI+): $\mathrm{m} / z$ calculated for $\mathrm{C}_{35} \mathrm{H}_{59} \mathrm{BrNaO}_{7} \mathrm{Si}[\mathrm{M}+\mathrm{Na}]^{+}$721.3106, found 721.3103.

(5S,7S,8R,E)-1-Bromo-8-((4R,6S)-6-(1-((tert-butyldimethylsilyl)oxy)-2-methylpropan-2-yl)-2,2dimethyl-1,3-dioxan-4-yl)-2-methoxy-7-((4-methoxybenzyl)oxy)-3-methylenenon-1-en-5-yl palmitate (2)

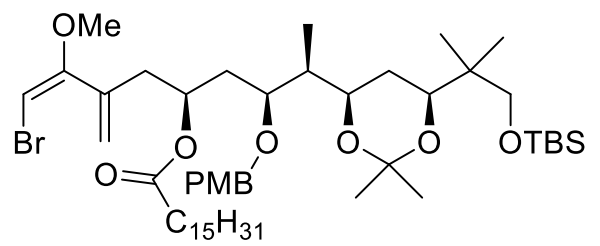

Palmitic acid (13 $\mathrm{mg}, 0.05 \mathrm{mmol})$ was added to a solution of $\mathrm{EDC} \cdot \mathrm{HCl}(18 \mathrm{mg}, 0.09 \mathrm{mmol}), \mathrm{Et}_{3} \mathrm{~N}(0.013$ $\mathrm{mL}, 0.09 \mathrm{mmol})$ and DMAP (11 $\mathrm{mg}, 0.09 \mathrm{mmol})$ in $\mathrm{CH}_{2} \mathrm{Cl}_{2}(0.35 \mathrm{~mL})$ and the solution was stirred for 20 minutes. 1 (22 $\mathrm{mg}, 0.031 \mathrm{mmol})$ dissolved in $\mathrm{CH}_{2} \mathrm{Cl}_{2}(0.15 \mathrm{~mL})$ was added and the solution was stirred for $16 \mathrm{~h}$. The reaction was quenched with saturated solution of $\mathrm{NH}_{4} \mathrm{Cl}(1 \mathrm{~mL})$ and extracted three times with AcOEt. The organic layer was dried over $\mathrm{MgSO}_{4}$, filtered, evaporated under reduced pressure and purified by silica gel column chromatography with hexane-EtOAc 95:5 to obtain 2 as a colorless oil $\left(12 \mathrm{mg}\right.$, cuant. brsm) and $2(14 \mathrm{mg})$. $[\alpha]_{\mathrm{D}}=+1.86$ (c 0.5 , $\mathrm{CH}_{2} \mathrm{Cl}_{2}$ ). IR (KBr film) v 2961, 2926, 2848, 1740, 1612, 1511, 1456, 1251, $1089 \mathrm{~cm}^{-1} .{ }^{1} \mathrm{H}$ NMR $\left(400 \mathrm{MHz}, \mathrm{CDCl}_{3}\right) \delta 0.01(\mathrm{~s}, 6 \mathrm{H}), 0.72(\mathrm{~s}, 3 \mathrm{H}), 0.74$ (s, 3H), 0.88 (brs, 12H), 0.94 (d, J = 7.0 $\mathrm{Hz}, 3 \mathrm{H}), 1.11-1.21$ (m, 2H), 1.25 (brs, 24H), 1.30 (s, 3H), 1.35 (s, 3H), $1.56-1.60$ (m, 2H), $1.66(\mathrm{td}, J=7.0,6.4,3.3 \mathrm{~Hz}, 1 \mathrm{H}), 1.82-1.95(\mathrm{~m}, 2 \mathrm{H}), 2.15-2.29(\mathrm{~m}, 2 \mathrm{H}), 2.54(\mathrm{qd}, J=14.2$, $6.4 \mathrm{~Hz}, 2 \mathrm{H}), 3.14(\mathrm{~d}, J=9.2 \mathrm{~Hz}, 1 \mathrm{H}), 3.44(\mathrm{~d}, J=9.2 \mathrm{~Hz}, 1 \mathrm{H}), 3.45-3.49(\mathrm{~m}, 1 \mathrm{H}), 3.55(\mathrm{~s}$, $3 \mathrm{H}), 3.65(\mathrm{dd}, J=11.2,2.9 \mathrm{~Hz}, 1 \mathrm{H}), 3.79(\mathrm{~s}, 3 \mathrm{H}), 3.79-3.79(\mathrm{~m}, 1 \mathrm{H}), 4.35(\mathrm{~d}, J=11.3 \mathrm{~Hz}$, 
1H), $4.47(\mathrm{~d}, J=11.3 \mathrm{~Hz}, 1 \mathrm{H}), 4.99(\mathrm{p}, J=6.7 \mathrm{~Hz}, 1 \mathrm{H}), 5.31(\mathrm{~s}, 1 \mathrm{H}), 5.34(\mathrm{~d}, J=1.4 \mathrm{~Hz}, 1 \mathrm{H})$, $5.41(\mathrm{~d}, J=1.4 \mathrm{~Hz}, 1 \mathrm{H}), 6.85(\mathrm{~d}, J=8.7 \mathrm{~Hz}, 2 \mathrm{H}), 7.25(\mathrm{~d}, J=8.7 \mathrm{~Hz}, 2 \mathrm{H}) .{ }^{13} \mathrm{C}-\mathrm{RMN}(100.6$ $\mathrm{MHz}, \mathrm{CDCl}_{3}$ ) $\delta$-5.6 (q), -5.5 (q), 9.9 (q), 14.1 (q), 18.3 (s), 18.9 (q), 19.8 (q), 20.9 (q), 22.7 (t), $25.0(\mathrm{t}), 25.9(\mathrm{q}), 28.5(\mathrm{t}), 29.3(\mathrm{t}), 29.3(\mathrm{t}), 29.4(\mathrm{t}), 29.5(\mathrm{t}), 29.7(\mathrm{t}), 30.2(\mathrm{~d}), 34.6(\mathrm{t}), 35.0(\mathrm{t})$, $38.5(\mathrm{~s}), 39.0(\mathrm{t}), 41.0(\mathrm{~d}), 55.2(\mathrm{q}), 55.6(\mathrm{q}), 68.7(\mathrm{t}), 69.9(\mathrm{~d}), 70.0(\mathrm{~d}), 70.2(\mathrm{t}), 71.4(\mathrm{~d}), 75.9$ (d), 78.7 (d), 97.9 (s), 113.7 (s), 122.0 (t), 129.2 (s), 131.0 (s), 138.4 (s), 158.5 (s), 159.0 (s), 172.9 (s). HRMS (ESI+): $\mathrm{m} / z$ calculated for $\mathrm{C}_{51} \mathrm{H}_{90} \mathrm{BrO}_{8} \mathrm{Si}[\mathrm{M}+\mathrm{H}]^{+}$937.5583, found 937.5559.

\section{Solvent optimization in transformation $5 \rightarrow 10$}<smiles>CC(C=O)C1CCOC(NC(=O)O)O1</smiles>

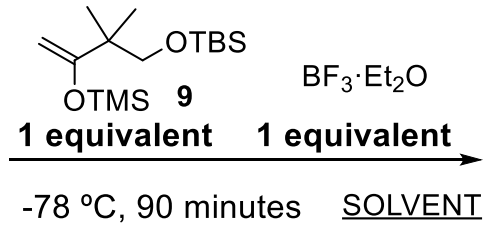<smiles>CC(CC(=O)C(C)(C)C[SeH])[C@@H](O)CC(=O)O</smiles>

1 equivalent

\begin{tabular}{cccc}
\hline Entry & Solvent & Yield (\%) & $\mathbf{1 0 ( 2 3 R ) : 1 0 ~ ( 2 3 S ) ~}$ \\
\hline 1 & $\mathrm{CH}_{2} \mathrm{Cl}_{2}$ & $78^{b}$ & $68: 32$ \\
2 & $\mathrm{CH}_{2} \mathrm{Cl}_{2}: \mathrm{Et}_{2} \mathrm{O}(1: 1)$ & $39^{c}$ & $100: 0$ \\
3 & $\mathrm{Et}_{2} \mathrm{O}$ & $0^{c}$ & -- \\
4 & $\mathrm{Toluene}^{c}$ & $84^{c, d}$ & $94: 6$ \\
$\mathbf{5}$ & $\mathbf{C H}_{\mathbf{2}} \mathbf{C l}_{\mathbf{2}}: \mathbf{E t}_{\mathbf{2}} \mathbf{O}(\mathbf{9 5 : 5 )}$ & $\mathbf{9 8}^{b}$ & $\mathbf{9 8 : 2}$ \\
\hline
\end{tabular}

All reactions were carried out with 1 equivalent of $\mathrm{BF} 3 \cdot \mathrm{OEt} 2$ in the described solvent at $-78{ }^{\circ} \mathrm{C}$ during 90 minutes. ${ }^{b}$ Yield was based in the recovery of $5\left(\mathrm{Y}_{\mathrm{brsm}}\right)$. Diasteromeric ratio was determined separating 10(5R) and 10(5S) via column cromatography. ${ }^{c}$ Yield and diasteromeric ratio were determined by integration of the ${ }^{1} \mathrm{H}$ NMR spectrum of the unpurified reaction mixture. ${ }^{d}$ The reaction crude had additional ${ }^{1} \mathrm{H}$ NMR signals non-related with $\mathbf{5 , 9}$ or $\mathbf{1 0}$.

\section{C23 configuration of 10 determination}

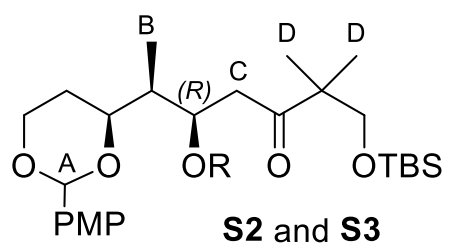

\begin{tabular}{c|c|c|c|c} 
& $\delta \mathrm{H}_{\mathrm{A}}$ & $\delta \mathrm{H}_{\mathrm{B}}$ & $\delta \mathrm{H}_{\mathrm{C}}$ & $\delta \mathrm{H}_{\mathrm{D}}$ \\
\hline $\mathbf{S 2}=R$-MPA & 5.05 & 0.86 & 2.86 & 1.05 \\
\hline $\mathbf{S 3}=S$-MPA & 5.31 & 0.99 & 2.74 & 0.92 \\
\hline$\Delta^{\mathrm{RS}}$ & -0.26 & -0.13 & +0.12 & +0.13
\end{tabular}




\section{Solvent and temperature optimization in transformation $10 \rightarrow \mathrm{S} 4$}

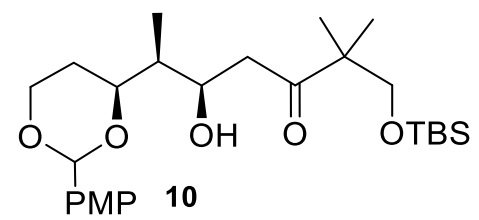

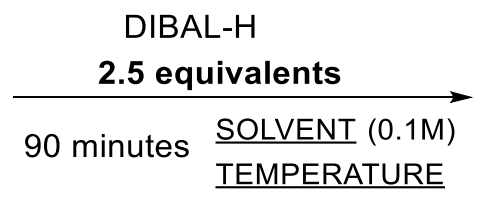<smiles>[Y16]C1OCC[C@@H]([C@@H](C)[C@@H](O)C[C@@H](O)C(C)(C)C[SeH])O1</smiles>

\begin{tabular}{cccc}
\hline Entry & Solvent & Temperature & S4(21R):S4(21S) \\
\hline 1 & $\mathrm{CH}_{2} \mathrm{Cl}_{2}$ & $-78^{\circ} \mathrm{C}$ & $52: 48$ \\
2 & $\mathrm{Et}_{2} \mathrm{O}$ & $-78^{\circ} \mathrm{C}$ & $55: 45$ \\
3 & $\mathrm{THF}$ & $-78^{\circ} \mathrm{C}$ & $65: 35$ \\
$\mathbf{4}$ & $\mathbf{T H F}$ & $\mathbf{- 1 0 0}{ }^{\circ} \mathrm{C}$ & $\mathbf{8 4 : 1 6}$ \\
\hline
\end{tabular}

All reactions were carried out with 2.5 equivalents of DIBAL-H the described solvent at the described temperature for 90 minutes. All the starting material was consumed after the reaction time. Diasteromeric ratio was determined by integration of the ${ }^{1} \mathrm{H}$ NMR spectrum of the unpurified reaction mixture.

\section{C19 configuration of 13 determination}

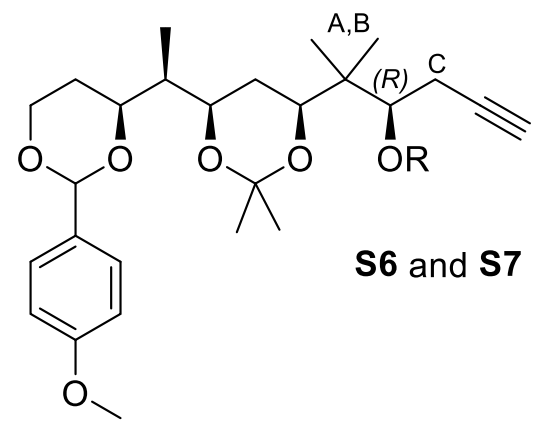

\begin{tabular}{c|c|c|c} 
& $\delta \mathrm{H}_{\mathrm{A}}$ & $\delta \mathrm{H}_{\mathrm{B}}$ & $\delta \mathrm{H}_{\mathrm{C}}$ \\
\hline $\mathbf{S 7}=R$-MPA & $0.62 \mathrm{ppm}$ & $0.62 \mathrm{ppm}$ & $2.51 \mathrm{ppm}$ \\
\hline $\mathbf{S 6}=S$-MPA & $0.80 \mathrm{ppm}$ & $0.83 \mathrm{ppm}$ & $2.37 \mathrm{ppm}$ \\
\hline$\Delta^{\mathrm{RS}}$ & $-0,18$ & $-0,21$ & $+0,14$
\end{tabular}




\section{C27 Configuration of $\mathbf{1 6}$ determination}<smiles>[Y15]O[C@H](C[C@H](O)CC(=O)/C(=C\Br)OC)[C@@H](C)[C@H]1C[C@H](C(C)(C)C[OH2+])OC(C)(C)O1</smiles><smiles>CO/C(=C/Br)C(=O)C[C@H]1C[C@@H]([C@H](C)[C@H]2C[C@H](C(C)(C)C[SeH2])OC(C)(C)O2)O[C@@H](P)O1</smiles><smiles>[R16]C1CC([R2])OC([R16])O1</smiles>

1H NMR of S8

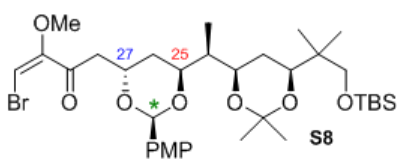

NOE irradiation at $*$

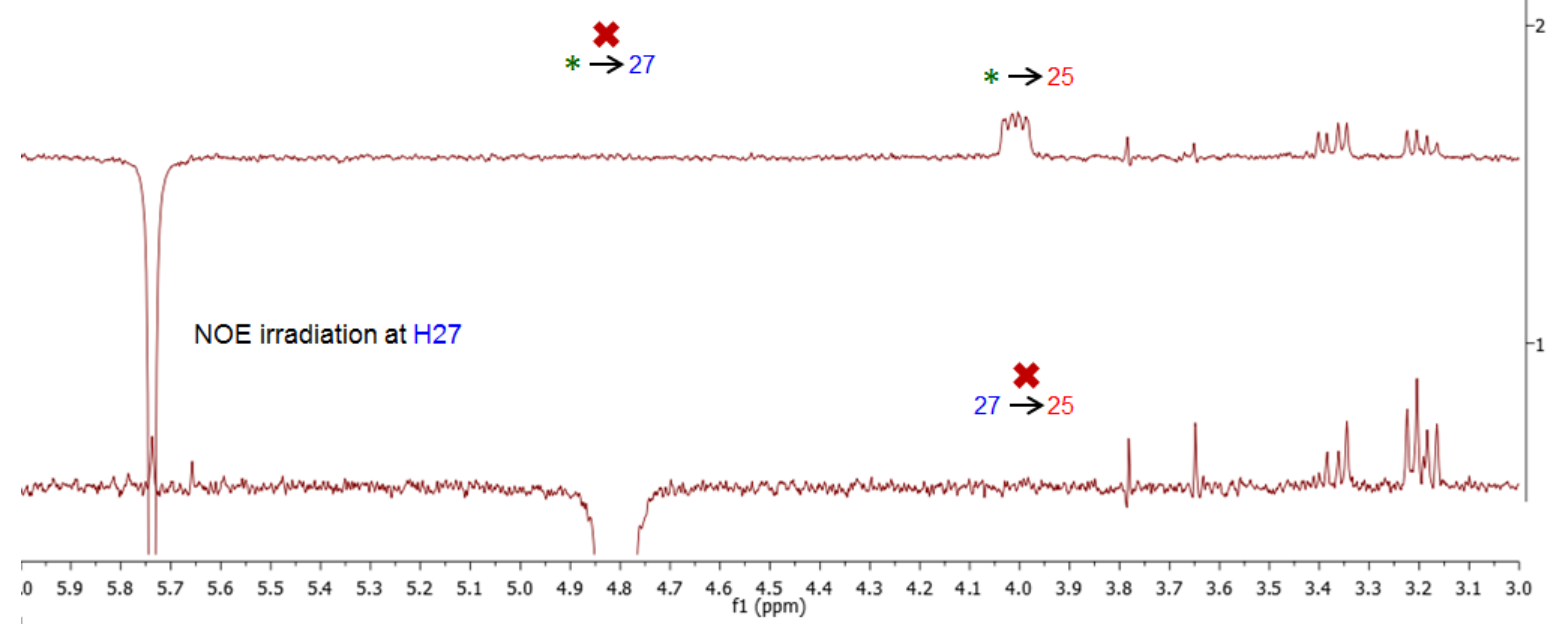

27

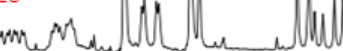


8. Table 1. $\mathrm{H}^{1}$ - and $\mathrm{C}^{13}$-NMR data of compound 1 and oscillariolide ${ }^{4}$
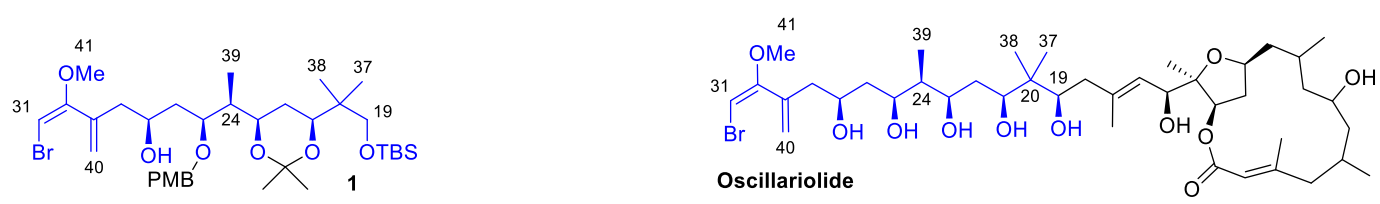

\begin{tabular}{c|ccc|ccc}
\hline Position & $\begin{array}{c}\delta^{1} \mathrm{H}(\mathbf{O s c}) \\
\mathrm{d}^{6}-\mathrm{DMSO}{ }^{4}\end{array}$ & $\begin{array}{c}\delta^{1} \mathrm{H}(\mathbf{1}) \\
\mathrm{d}^{6}-\mathrm{DMSO}\end{array}$ & $\begin{array}{c}\delta^{1} \mathrm{H}(\mathbf{1}) \\
\mathrm{CDCl}_{3}\end{array}$ & $\begin{array}{c}\delta^{13} \mathrm{C}(\mathbf{O s c}) \\
\mathrm{d}^{6}-\mathrm{DMSO}^{4}\end{array}$ & $\begin{array}{c}\delta^{13} \mathrm{C}(\mathbf{1}) \\
\mathrm{d}^{6}-\mathrm{DMSO}\end{array}$ & $\begin{array}{c}\delta^{13} \mathrm{C}(\mathbf{1}) \\
\mathrm{CDCl}_{3}\end{array}$ \\
\hline 20 & -- & -- & -- & 41.3 & 38.1 & 38.5 \\
24 & 1.44 & 1.57 & 1.79 & 40.7 & 40.3 & 40.4 \\
28 & $2.21 ; 2.38$ & $2.25 ; 2.45$ & 2.40 & 42.6 & 43.0 & 42.8 \\
29 & -- & -- & -- & 139.9 & 139.8 & 139.5 \\
30 & -- & -- & -- & 158.7 & 158.5 & 159.0 \\
31 & 5.60 & 5.63 & 5.32 & 79.1 & 79.1 & 78.6 \\
37 & 0.69 & 0.68 & 0.76 & 18.2 & 18.9 & 19.2 \\
38 & 0.82 & 0.70 & 0.78 & 18.7 & 20.5 & 20.7 \\
39 & 0.79 & 0.83 & 0.94 & 6.8 & 9.6 & 10.8 \\
40 & $5.22 ; 5.32$ & $5.25 ; 5.33$ & 5.37 & 120.3 & 120.4 & 121.9 \\
41 & 3.53 & 3.53 & 3.57 & 55.8 & 55.8 & 55.6 \\
\hline
\end{tabular}

9. Table 2. $H^{1}$ - and $C^{13}$-NMR data of compound 2 and phormidolide $A^{5}$
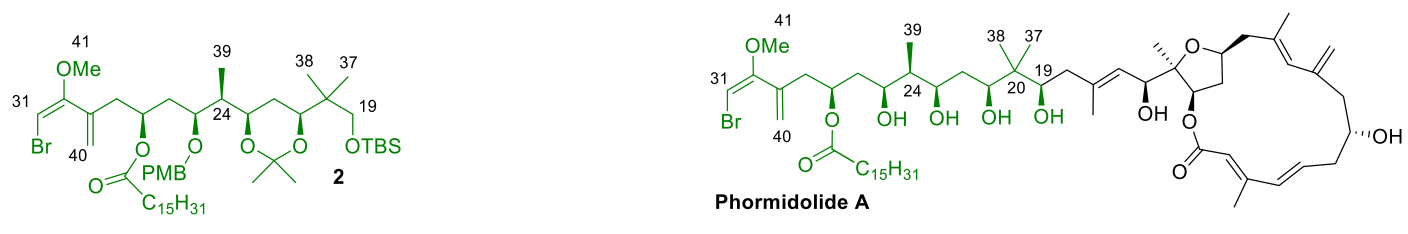

\begin{tabular}{c|cc|cc}
\hline Position & $\begin{array}{c}\delta^{1} \mathrm{H}(\mathbf{P h A}) \\
\mathrm{CDCl}^{5}\end{array}$ & $\begin{array}{c}\delta^{1} \mathrm{H}(\mathbf{2}) \\
\mathrm{CDCl}_{3}\end{array}$ & $\begin{array}{c}\delta^{13} \mathrm{C}(\mathbf{P h A}) \\
\mathrm{CDCl}_{3}{ }^{5}\end{array}$ & $\begin{array}{c}\delta^{13} \mathrm{C}(\mathbf{2}) \\
\mathrm{CDCl}_{3}\end{array}$ \\
\hline 20 & -- & -- & 40.4 & 38.5 \\
24 & 1.49 & 1.66 & 41.5 & 40.9 \\
28 & 2.57 & 2.54 & 39.3 & 39.0 \\
29 & -- & -- & 138.3 & 138.3 \\
30 & -- & -- & 158.4 & 158.5 \\
31 & 5.33 & 5.31 & 78.8 & 78.7 \\
37 & 0.91 & 0.74 & 13.7 & 18.9 \\
38 & 0.74 & 0.72 & 21.6 & 20.9 \\
39 & 0.92 & 0.94 & 5.0 & 9.8 \\
40 & $5.37 ; 5.42$ & $5.34 ; 5.41$ & 122.1 & 122.0 \\
41 & 3.59 & 3.55 & 55.6 & 55.6 \\
\hline
\end{tabular}




\section{NMR spectra of compounds}

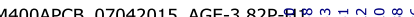

M400PCB / Num.Inv, AF/002630 ヘヘヘヘヘめ0

cdcl3 / Temp: 25C/N.Reg: XXXXXXXXXXX) |

Usuari: ad / Mostra: AGE-3.82P

Nom: ALEJANDRO GIL ESCOLANO

Data: 07/04/15 / Ope.: A.GIL<smiles>[Y6]C1OCC[C@@H]([C@@H](C)C(=O)N2C(=O)OC[C@H]2Cc2ccccc2)O1</smiles>

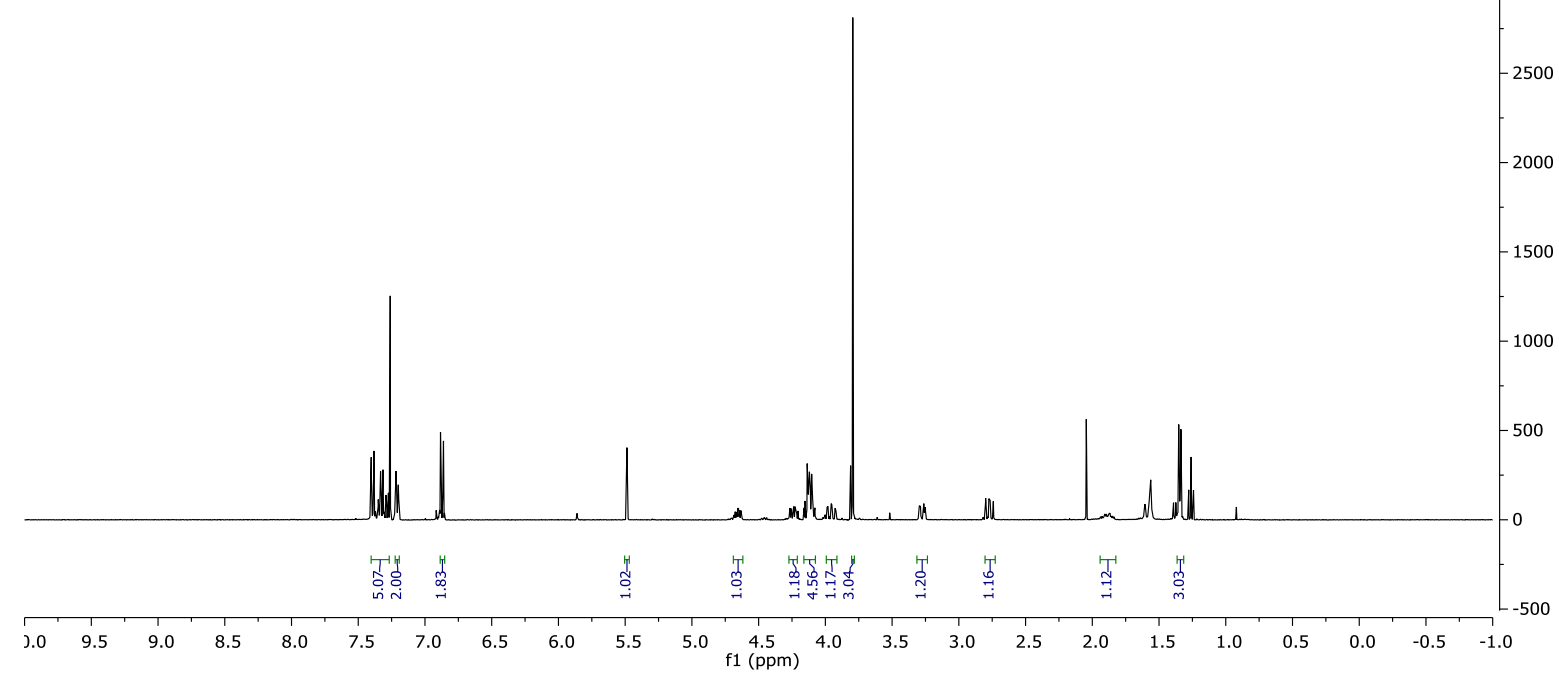

O00APCB 07042015 AGE-3.82P-C13 M400PCB / Num.Inv. AF/002630

cdcl3 / Temp: 25C / N.Reg: XXXXXXXXXX

Usuari: ad / Mostra: AGE-3.82P

Nom: ALEJANDRO GIL ESCOLANO

$$
\underbrace{O}_{P M P}
$$

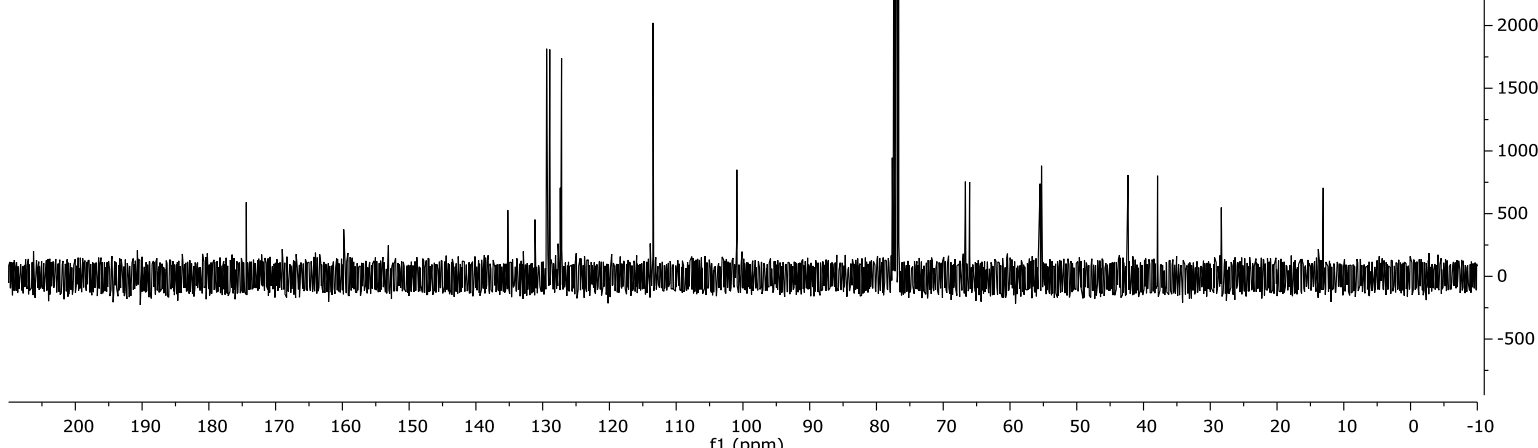



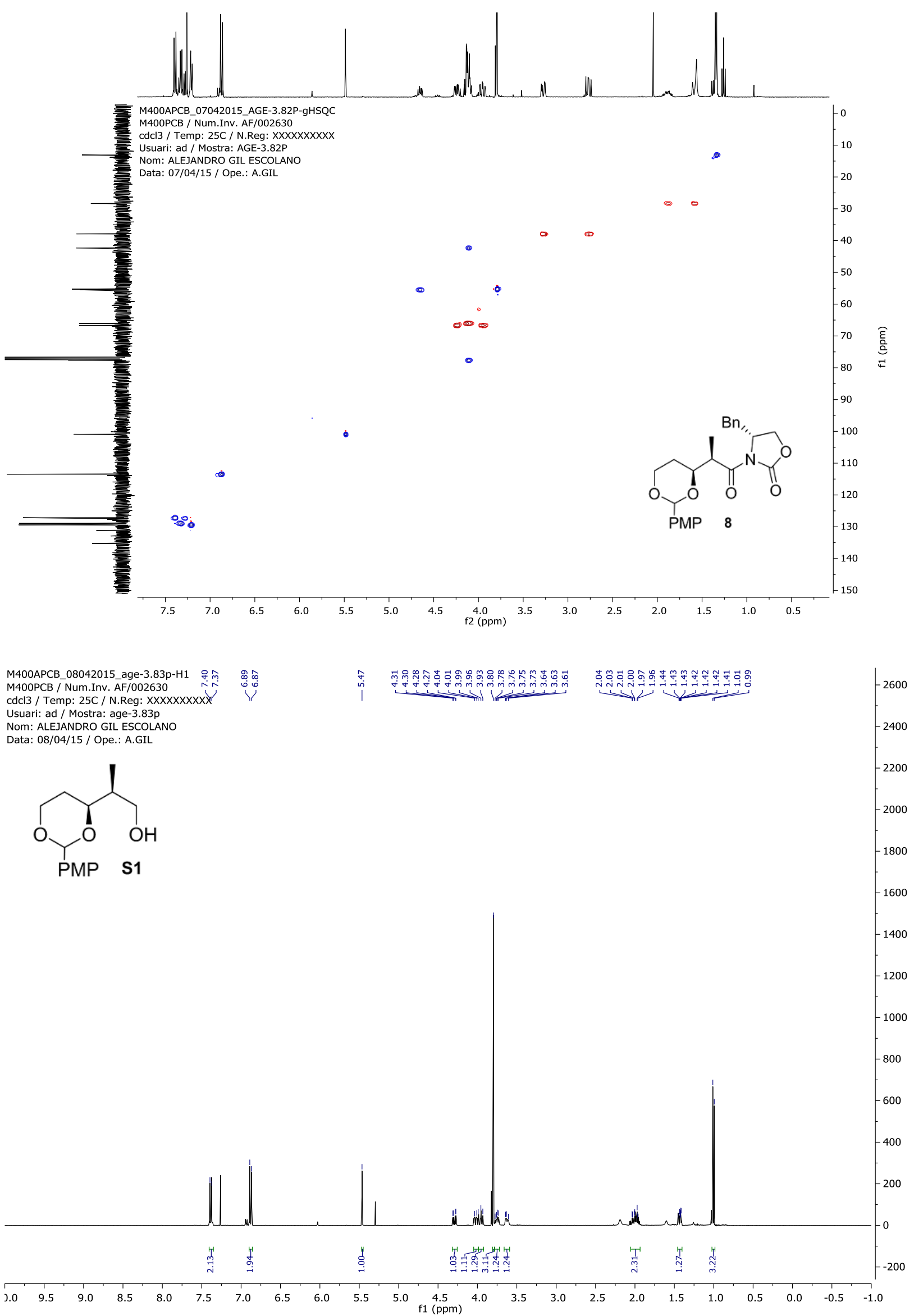
M400APCB_08042015_age-3.83p-C13

M400PCB / Num.Inv. AF/002630

cdcl3 / Temp: 25C/N.Reg: XXXXXXXXXX

Usuari. ad / Mostra. age-3.83p

Nom: ALEJANDRO GIL ESCOLANO

Data: 08/04/15 / Ope.: A.GIL
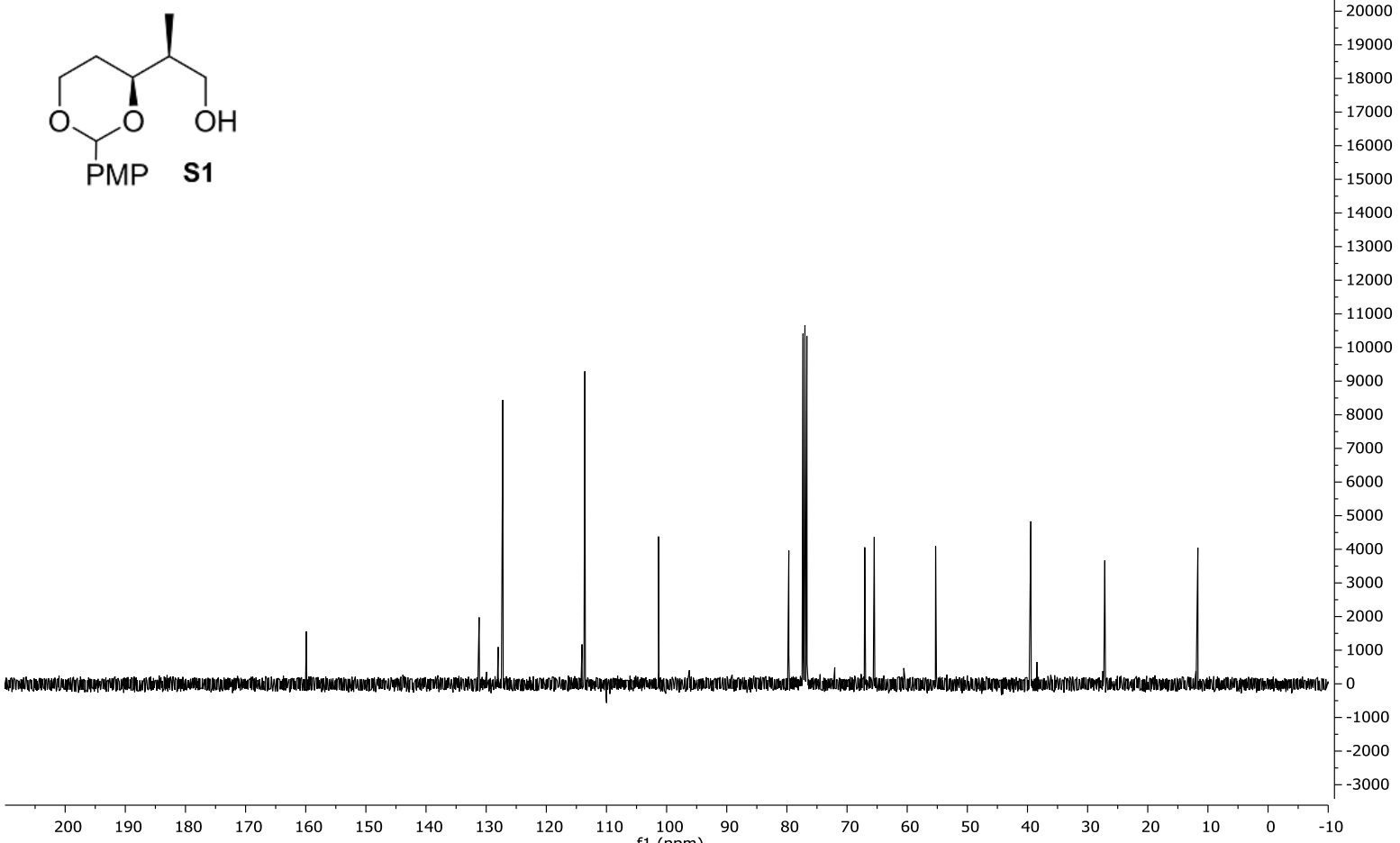

$\begin{array}{llllllllllllllllllllll}200 & 190 & 180 & 170 & 160 & 150 & 140 & 130 & 120 & 110 & \begin{array}{c}100 \\ \mathrm{f} 1(\mathrm{ppm})\end{array} & 90 & 80 & 70 & 60 & 50 & 40 & 30 & 20 & 10 & 0 & -10\end{array}$

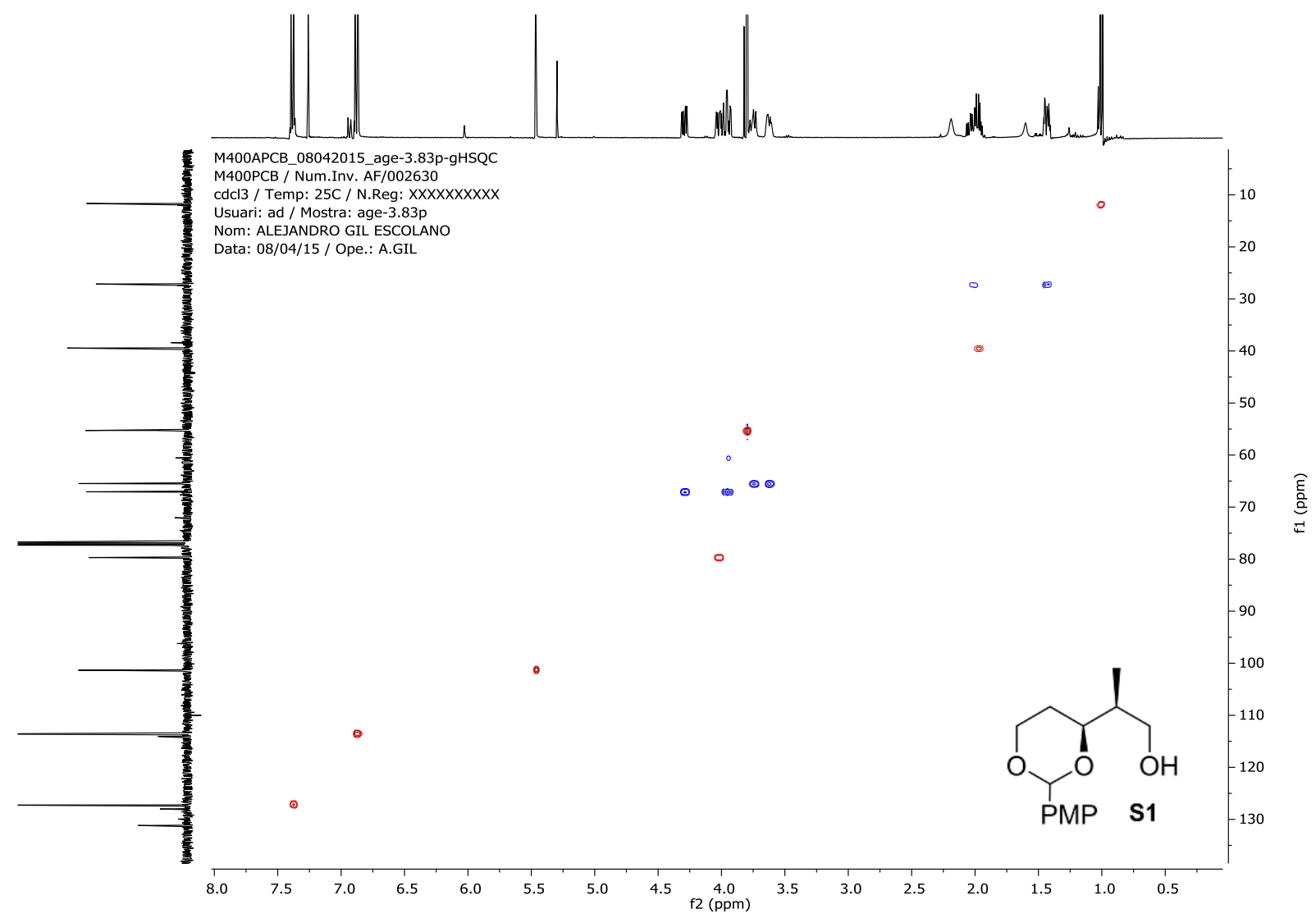


188490APCB_10042015_AGE-3.86P-H1_18 M400PCB / Num.Inv. AF/002630

रे

25C/N.Reg: $X X X X X$

Nom: ALEJANDRO GIL ESCOLANO

Data: 10/04/15 / Ope.: A.GIL

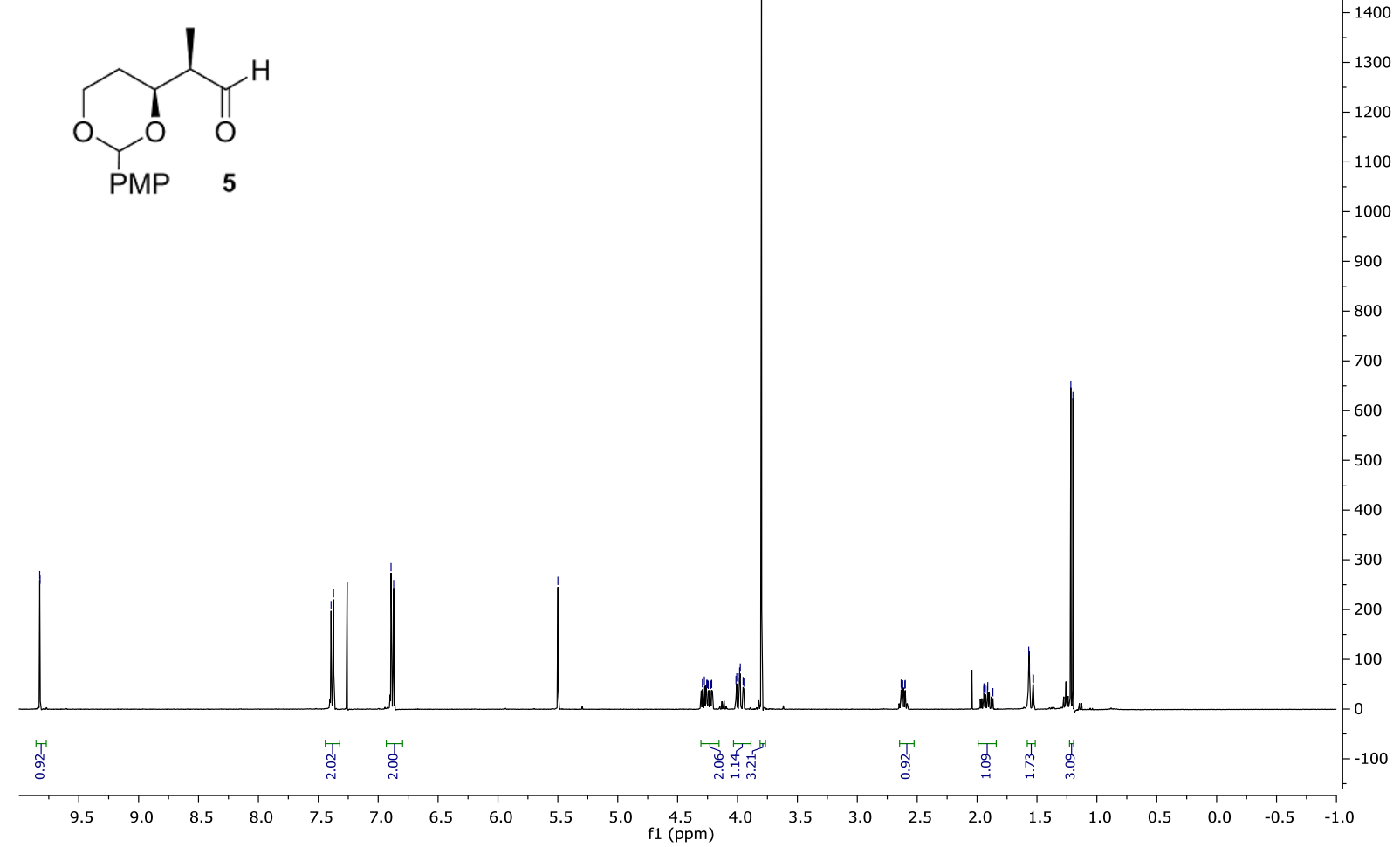

M400APCB 10042015 AGE-3.86P-C13 M400PCB / Num.Inv. AF/002630

eg: XXXXXXXXXX

a: AGE-3.86P

GIL ESCOLANO

Data: 10/04/15 / Ope.: A.GIL

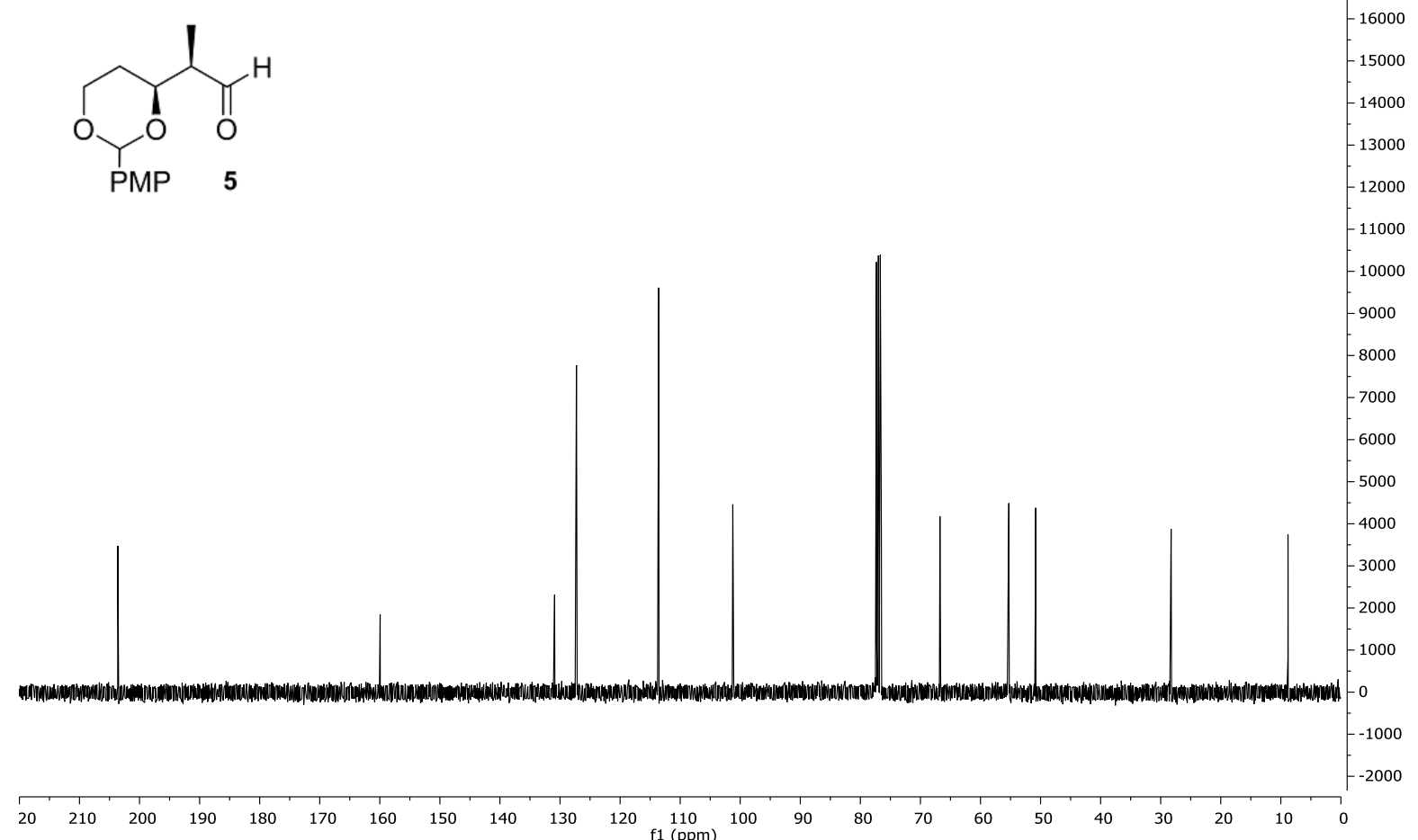




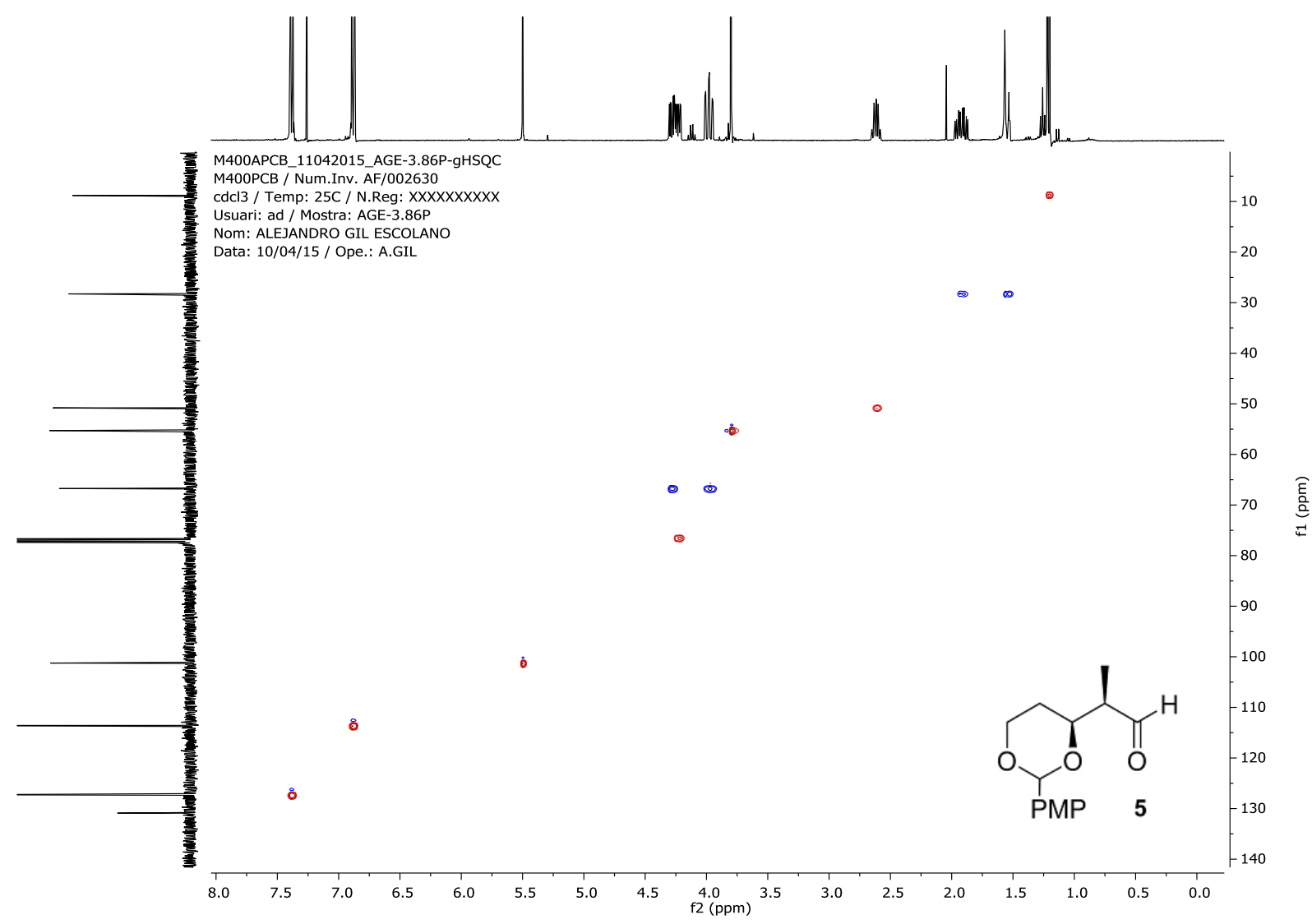

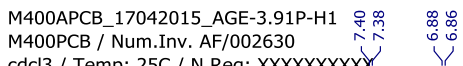

cdd13/ Temp: 25C / N.Reg: XXXXXXXXXX

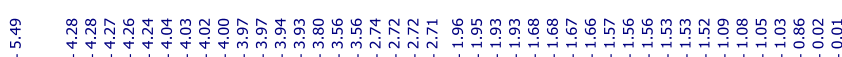

Udcl3/Temp: 25C/N.Reg: XXXX ad / Mostra: AGE-3.91P

Nom: ALEJANDRO GIL ESCOLANO

Data: $17 / 04 / 15$ / Ope.: A.GIL<smiles>C[C@H]([C@@H](O)CC(=O)C(C)(C)C[SeH])[C@@H](C)CO[SbH3]</smiles>

\section{$1 \underbrace{2}$}

$-7000$

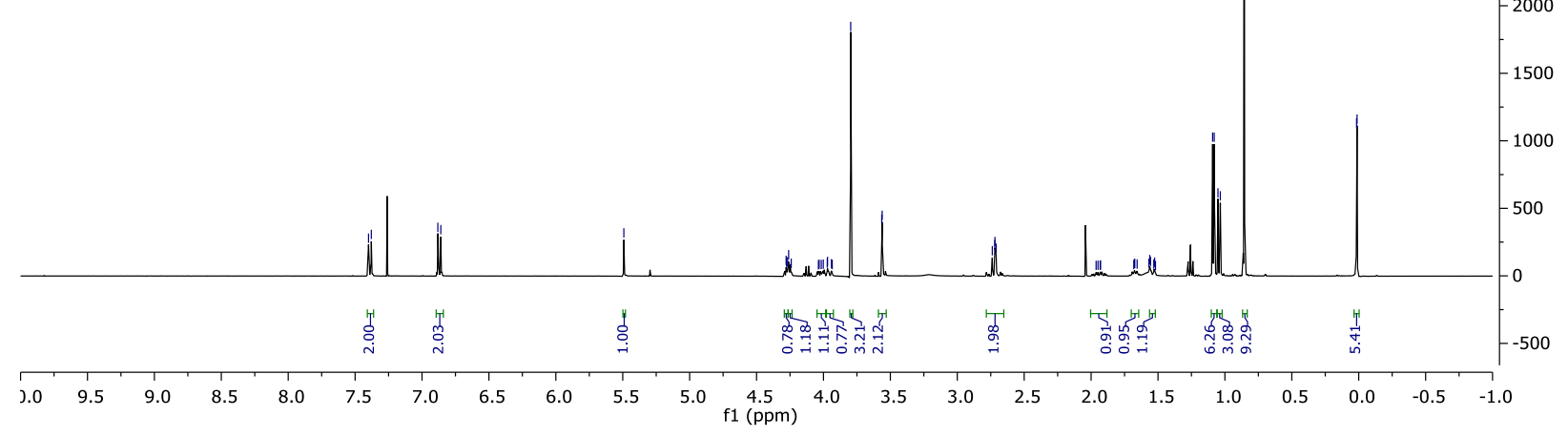


M400APCB_17042015 AGE-3.91P-C13

M400PCB / Num.Inv. AF/002630

cdcl3 / Temp: 25C / N.Reg: XXXXXX

Usuari: ad / Mostra: AGE-3.91P

Data: 17/04/15 / Ope.: A.GIL<smiles>C[C@@H]([C@@H]1CCOC([Y16]#N)O1)[C@H](O)CC(=O)C(C)(C)C[SeH]</smiles>
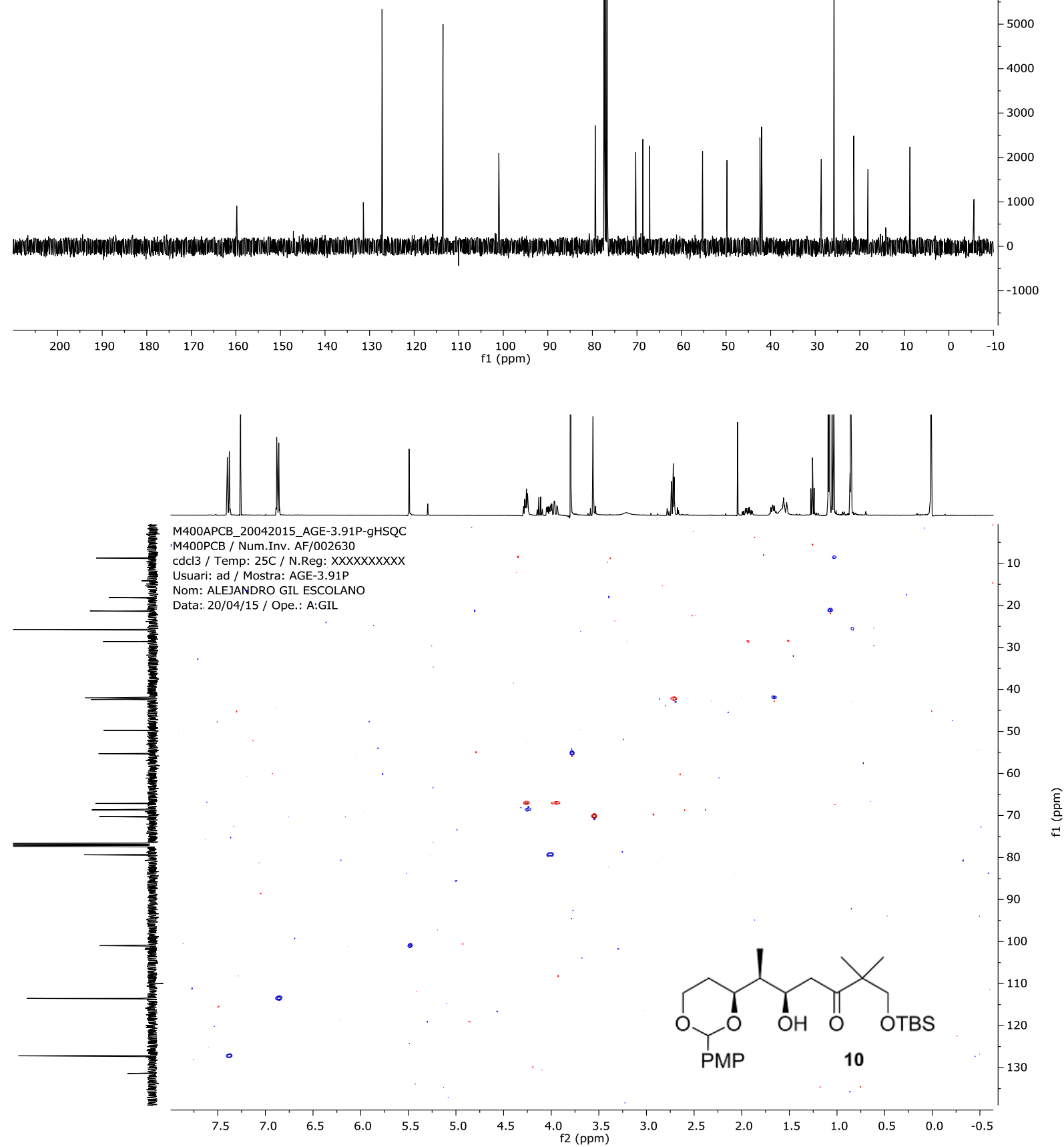


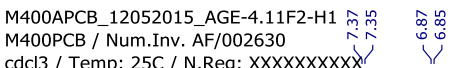

Udcl3 / Temp: $25 C /$ N.Reg: $X X X X X X$

Nom: ALEJANDRO GIL ESCOLANO

Data: 12/05/15 / Ope.: A.GIL

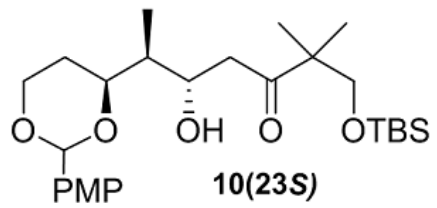

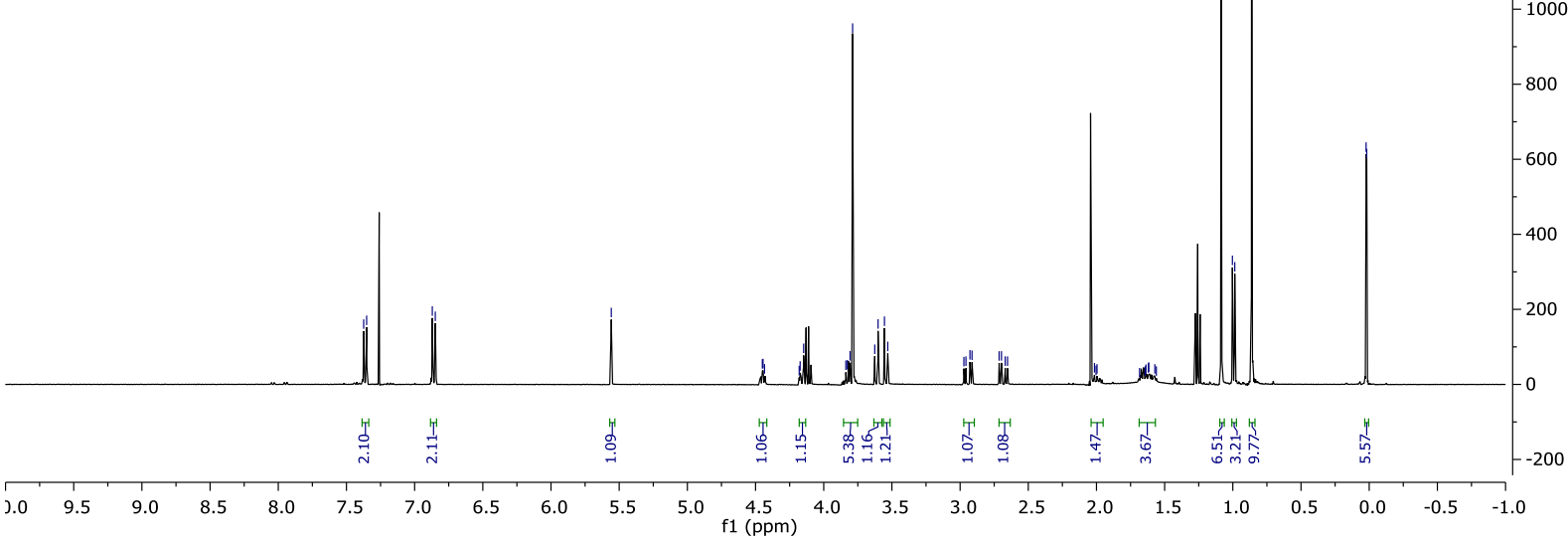

M400APCB_13052015_AGE-4.11F2-C13

M400PCB / Num.Inv. AF/002630

cdcl3 / Temp: 25C / N.Reg: XXXXXXXXXX

Usuari: ad / Mostra: AGE-4.11F2

Nom: ALEJANDRO GIL ESCOLANO

Data: 12/05/15 / Ope.: A.GIL<smiles>C[C@@H](CO[SbH3])[C@@H](C)[C@@H](O)CC(=O)C(C)(C)C[SeH]</smiles>

PMP 10(23S)

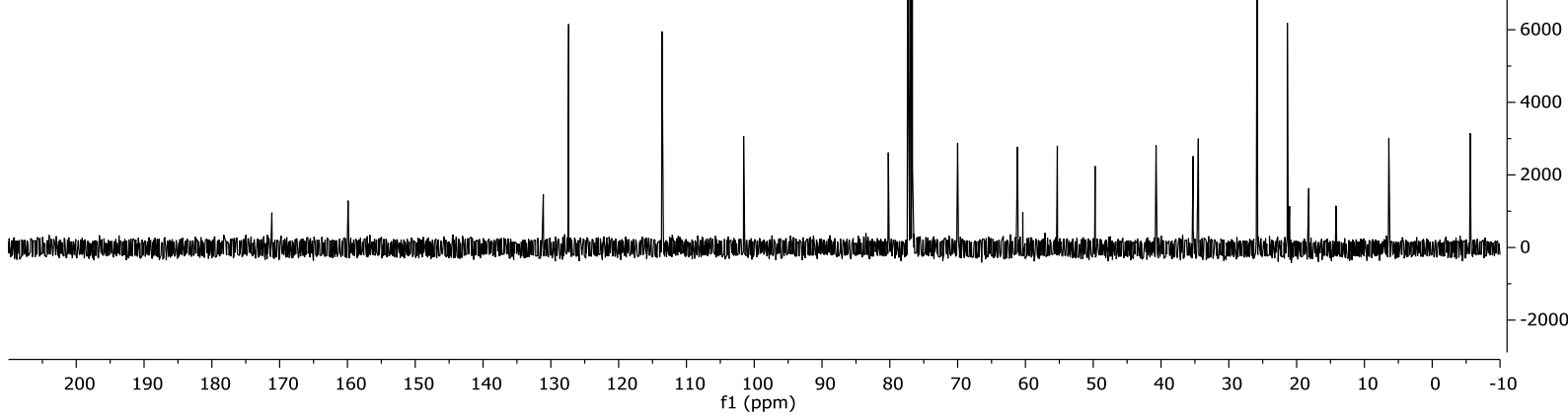




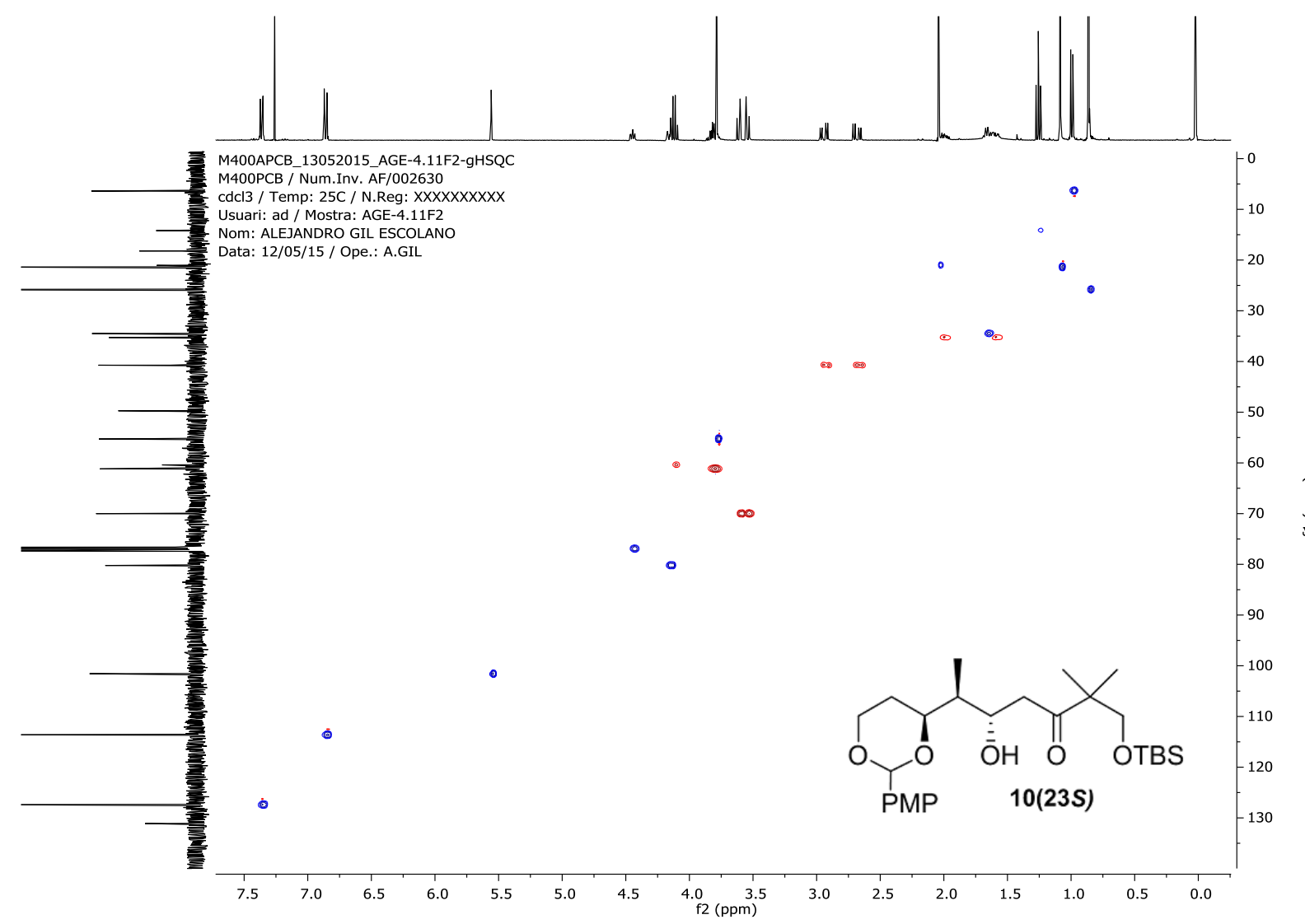

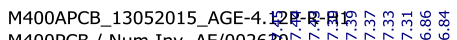

M400PCB / Num.Inv. AF/002630 N

cdcl3 / Temp: 25C/N.Reg: XXXXXXXXXX/

Usuari: ad / Mostra: AGE-4.12P-R

Nom: ALEJANDRO GIL ESCOLANO

Data: 13/05/15 / Ope.: A.GIL

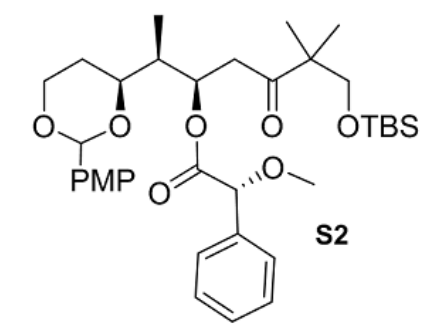

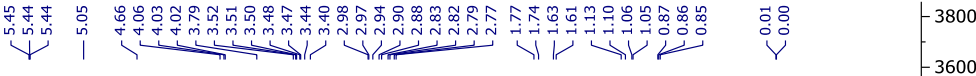

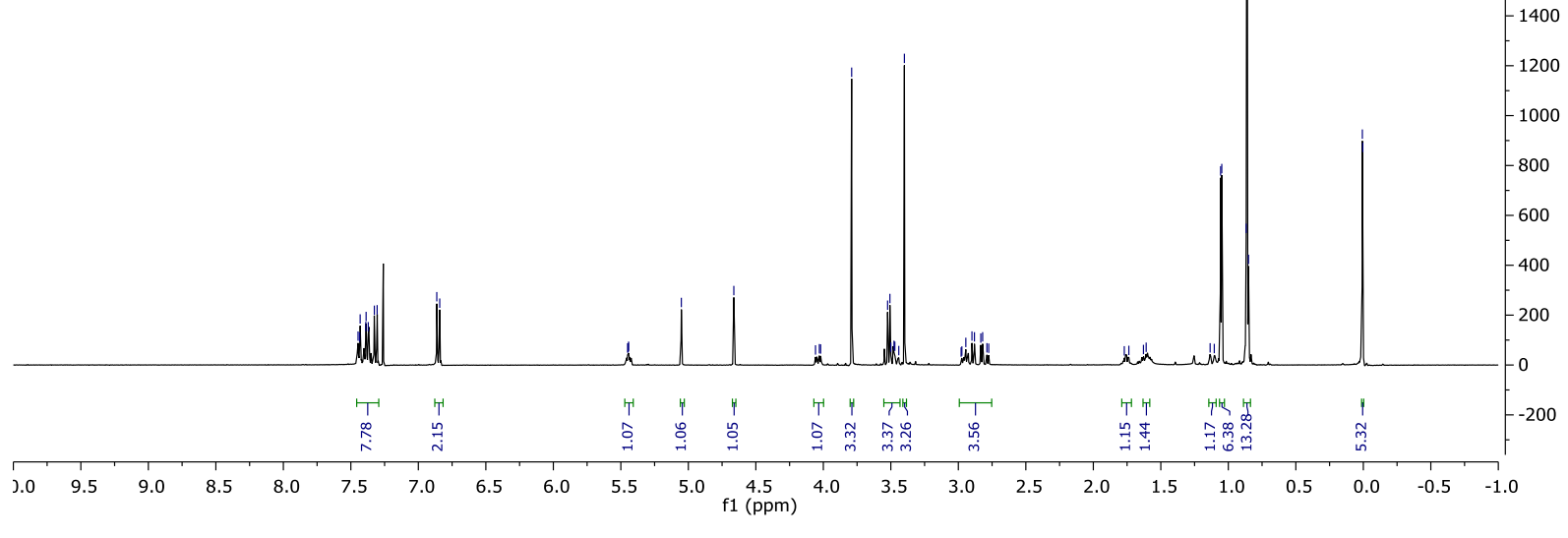


M400APCB_14052015 AGE-4.12P-R-C13 M400PCB / Num.Inv. AF/002630

cdcl3 / Temp: 25C / N.Reg: XXXXXXXXXXX

Usuari: ad / Mostra: AGE-4.12P-R

Data: 13/05/15 / Ope.: A.GIL
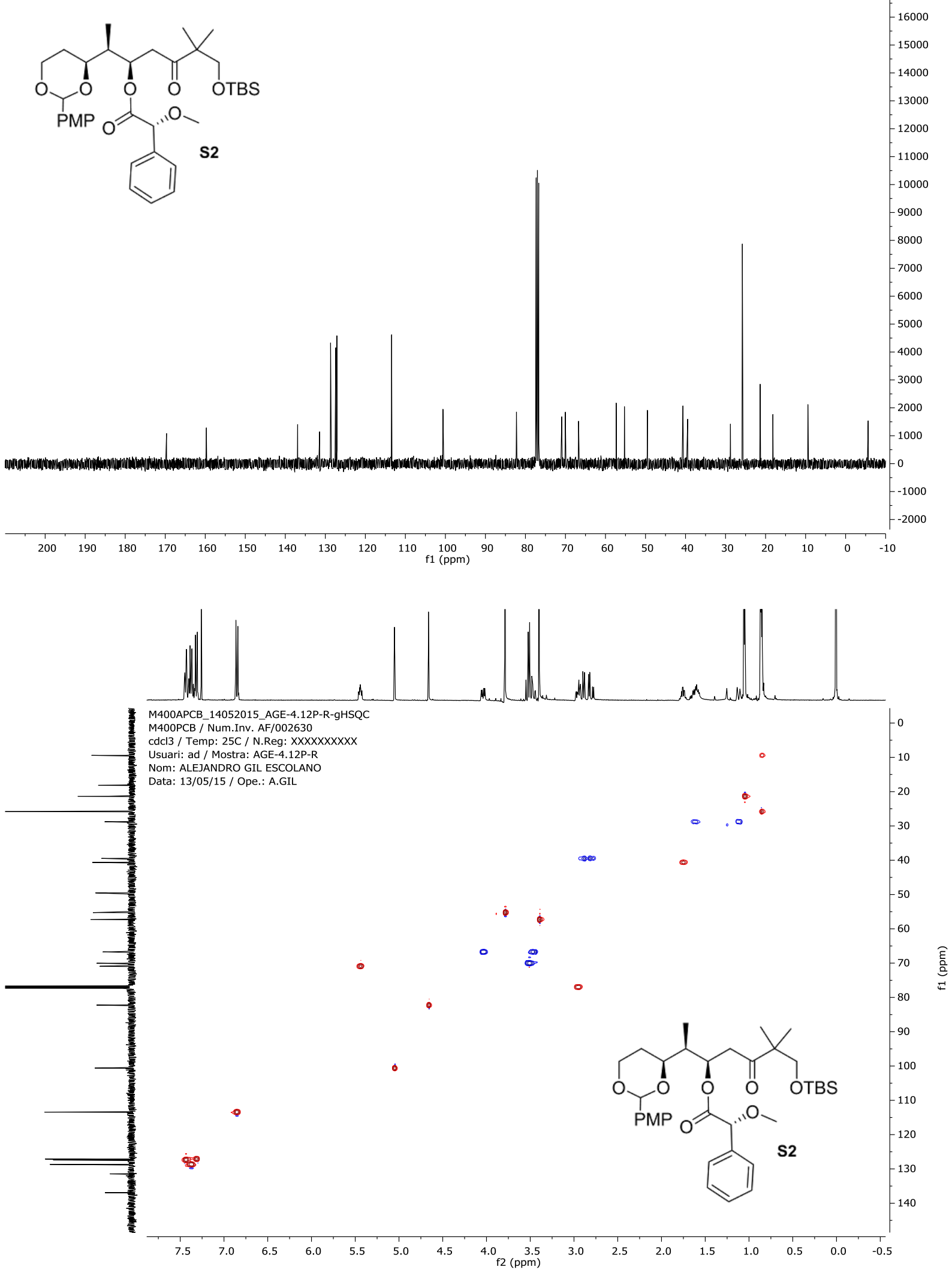


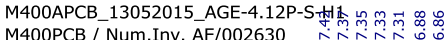

M400PCB / Num.Inv. AF/002630 N

cdcl3 / Temp: 25C / N.Reg: XXXXXXX

Usuari: ad / Mostra: AGE-4.12P-S

Data: $13 / 05 / 15 /$ Ope : A.GIL
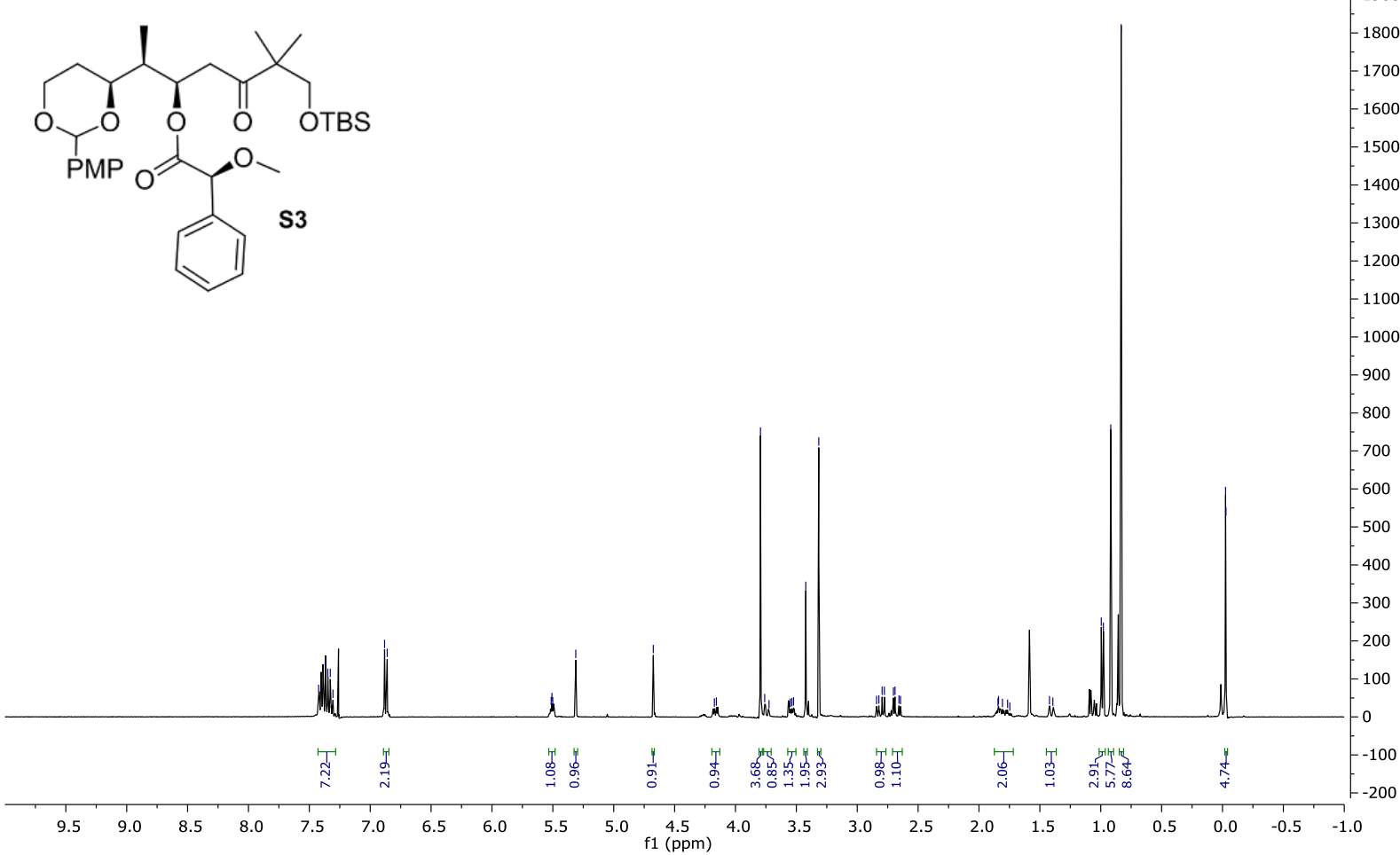

M400APCB 14052015_AGE-4_12P-S-C13 M400PCB / Num.Inv. AF/002630

Usuari: ad / Mostra: AGE-4_12P-S

Nom: ALEJANDRO GIL ESCOLANO

Data: 13/05/15/ Ope: A.GIL
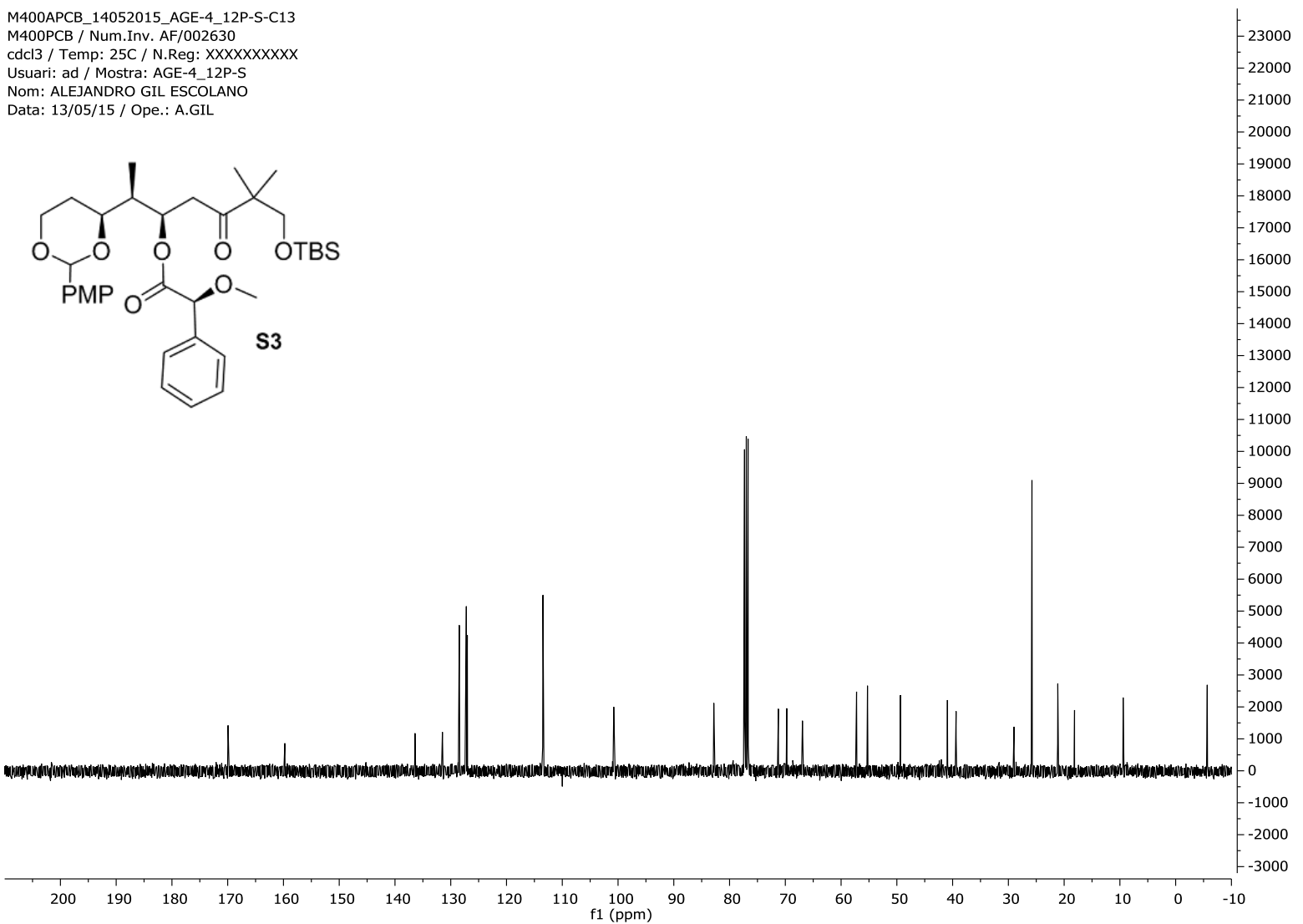


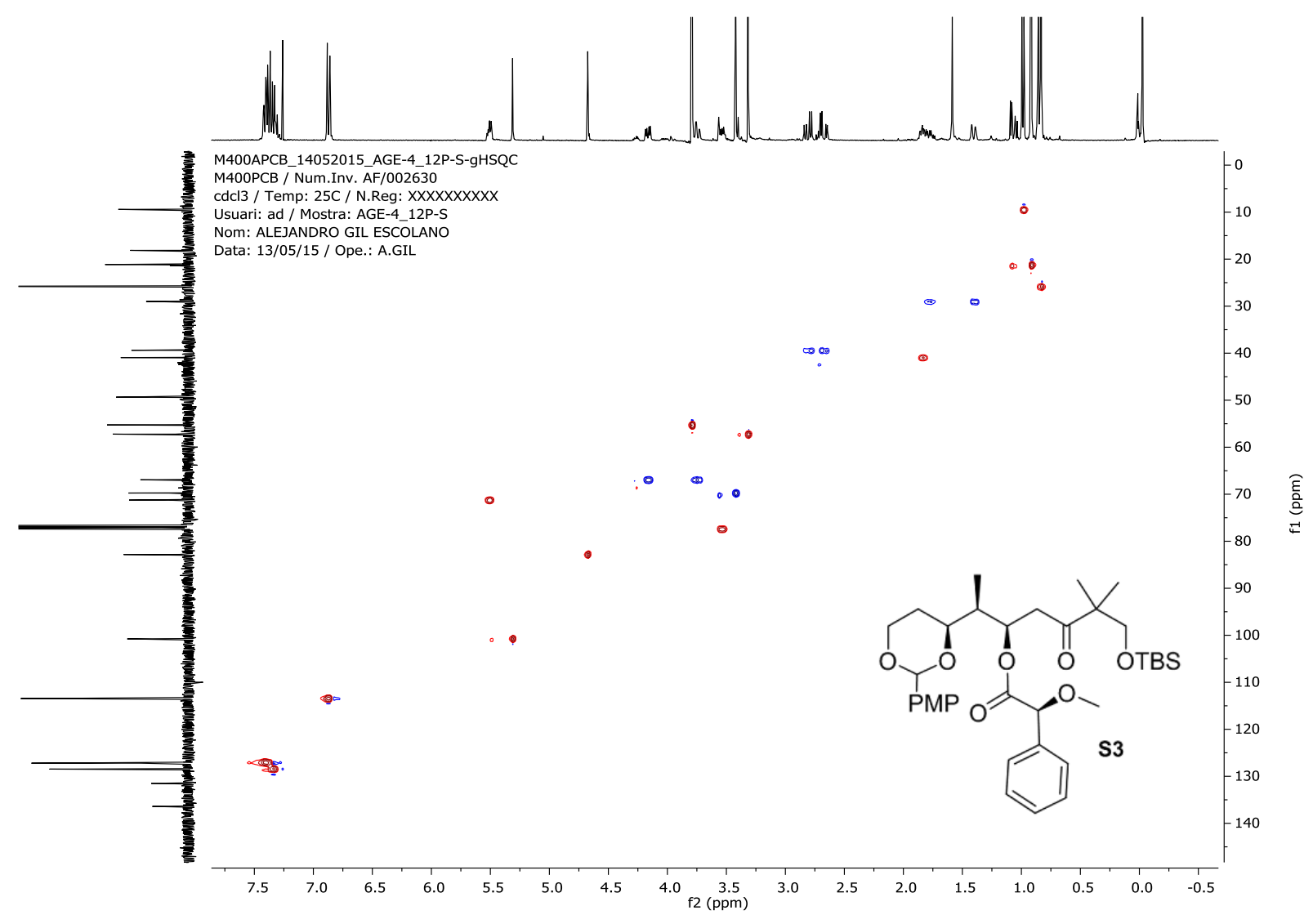

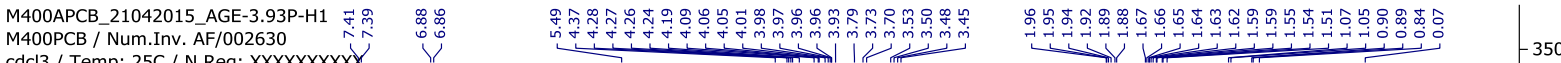
cdcl3 / Temp: 25C / N.Reg: XXXXXX

Usuari: ad / Mostra: AGE-3.93P

Nom: ALEJANDRO GIL ESCOLANO

Data: 21/04/15 / Ope.: A.GIL<smiles>CC(CC(O)C(C)(C)CO[18O])C1CCOC([18OH])O1</smiles>

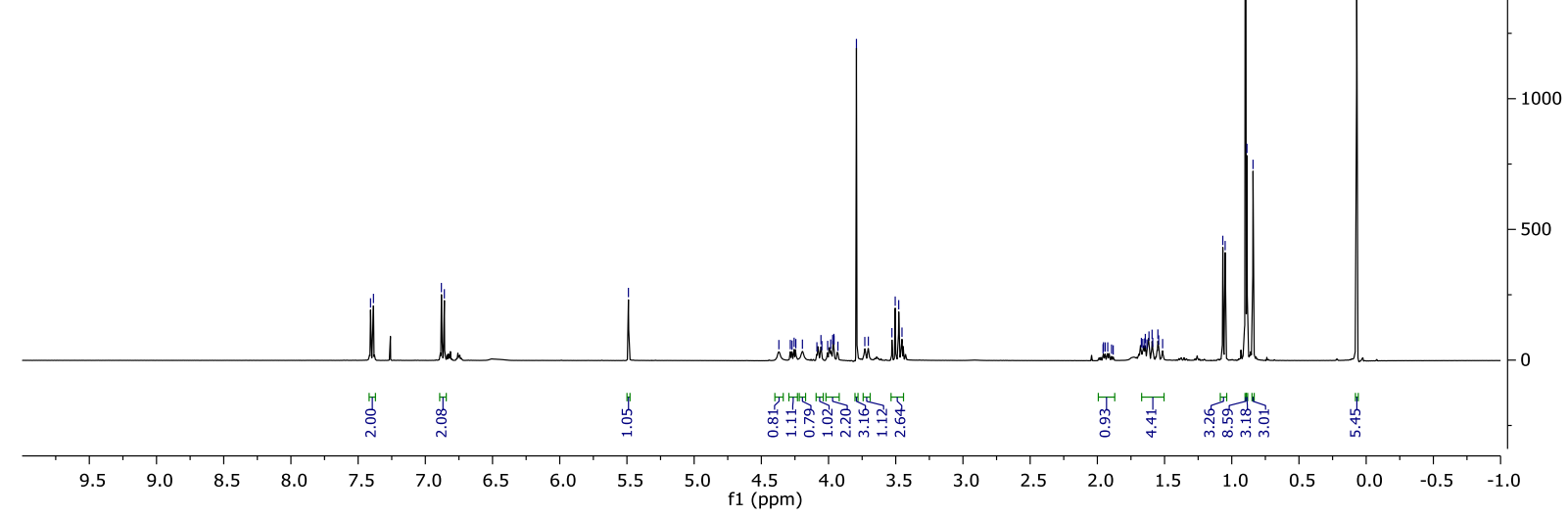


M400APCB 21042015 AGE-3.93P-C13

M400PCB / Num.Inv, AF/002630

cdcl3 / Temp: 25C / N.Reg: XXXXXXXXXXX

Usuari: ad / Mostra: AGE-3.93P

Nom: ALEJANDRO GIL ESCOLANO

Data: 21/04/15 / Ope.: A.GIL<smiles>C[C@H]([C@@H](O)C[C@@H](O)C(C)(C)CO[Sb])[C@@H](O)CO[Sb]</smiles>
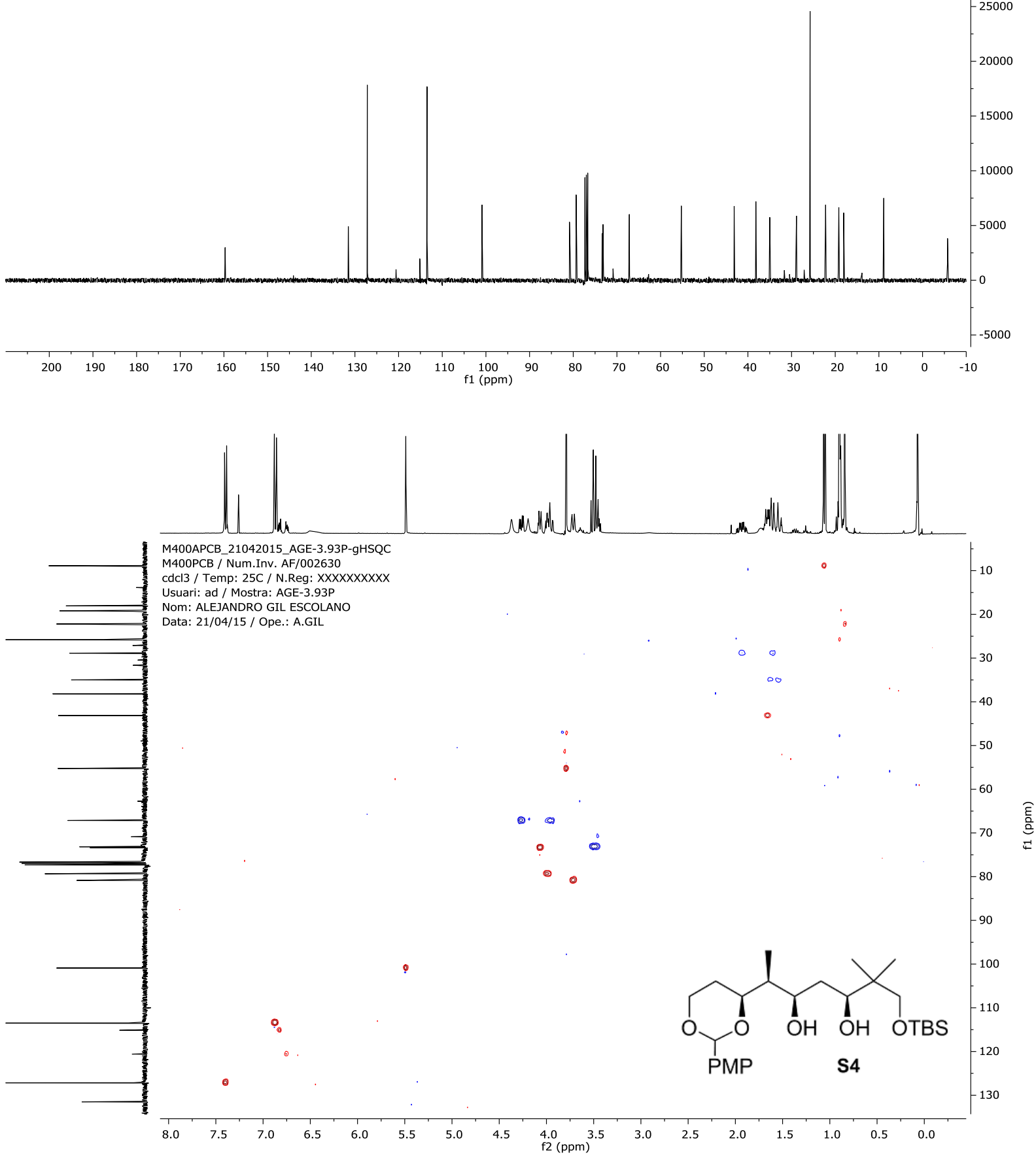

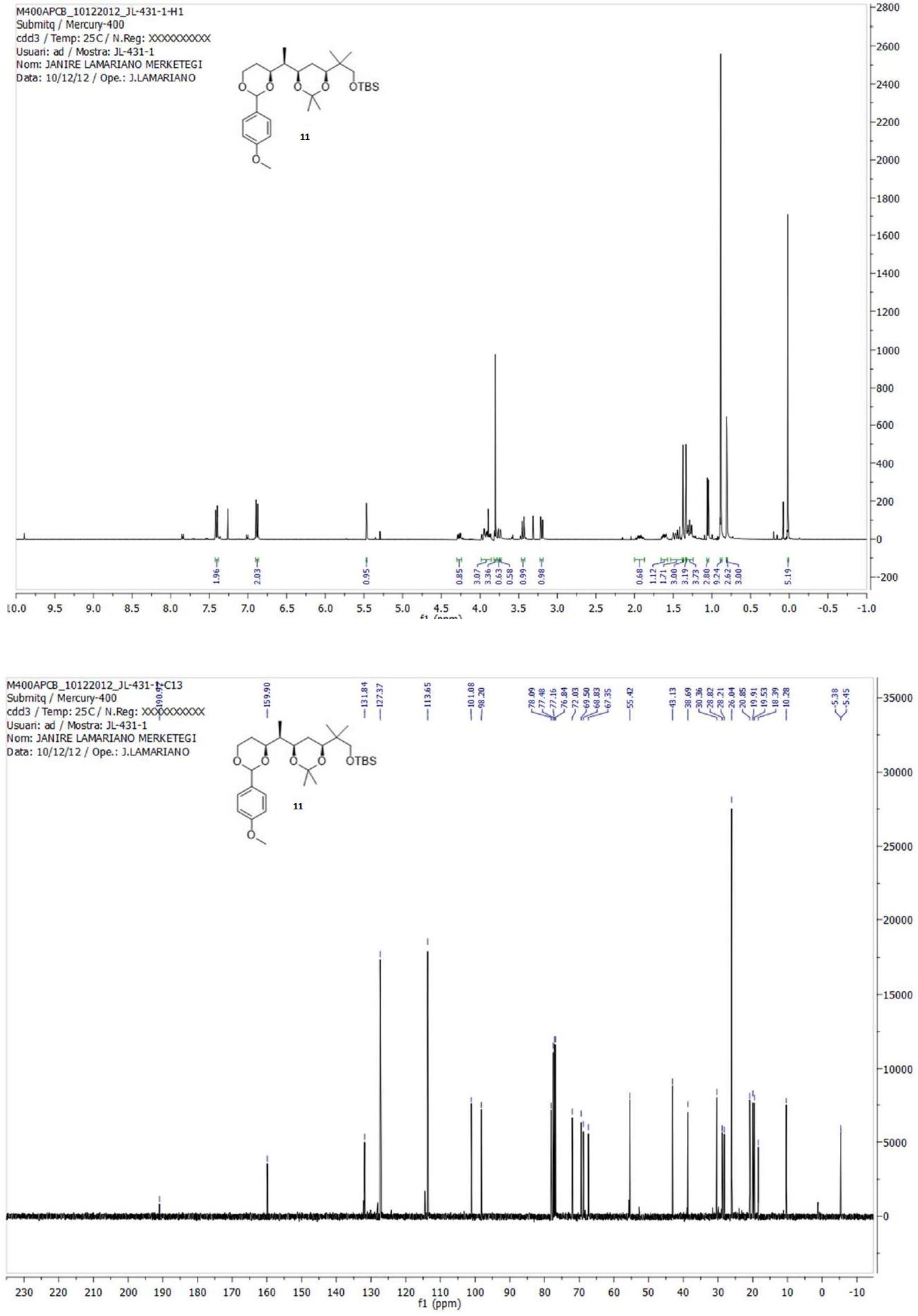

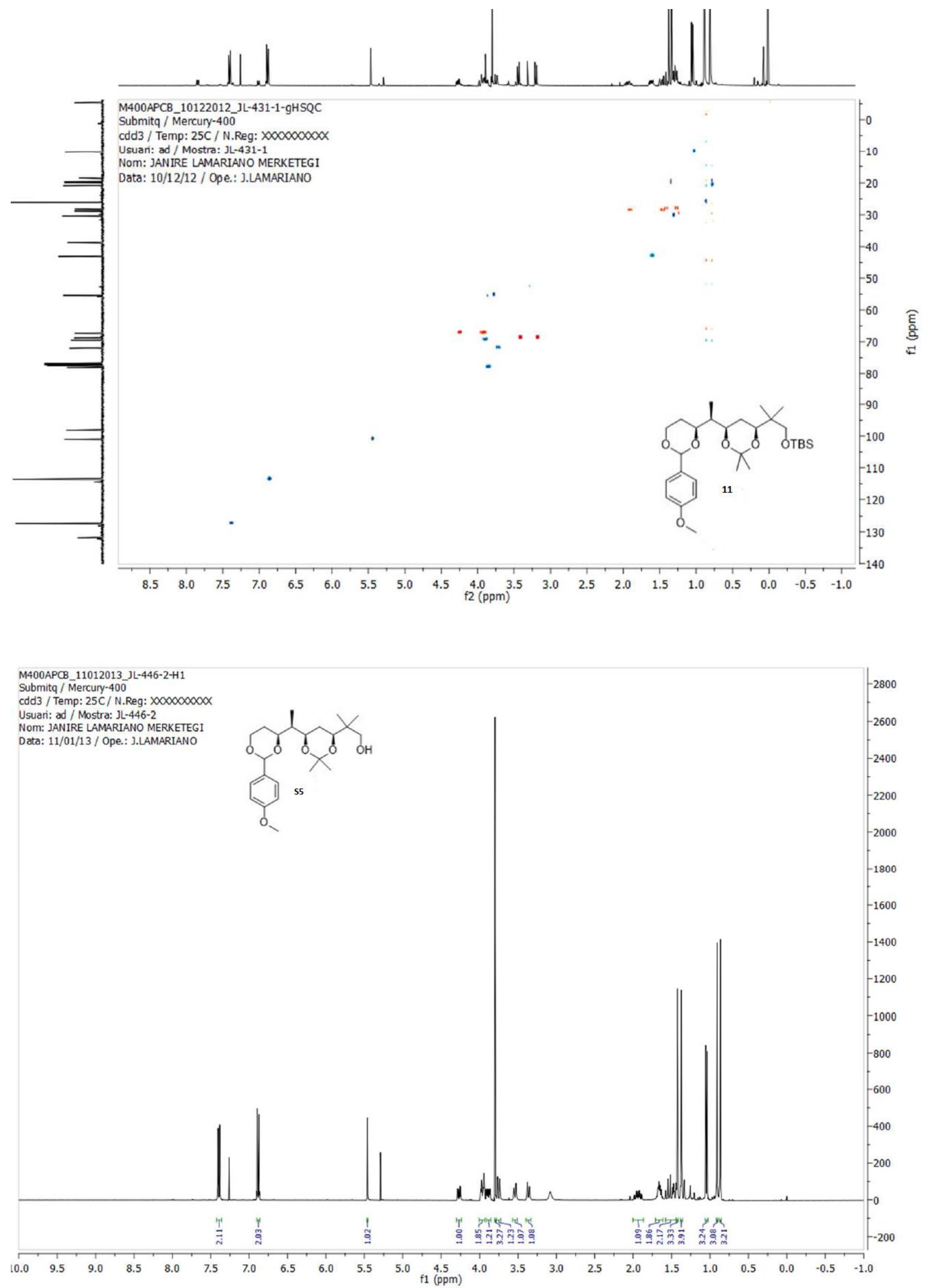

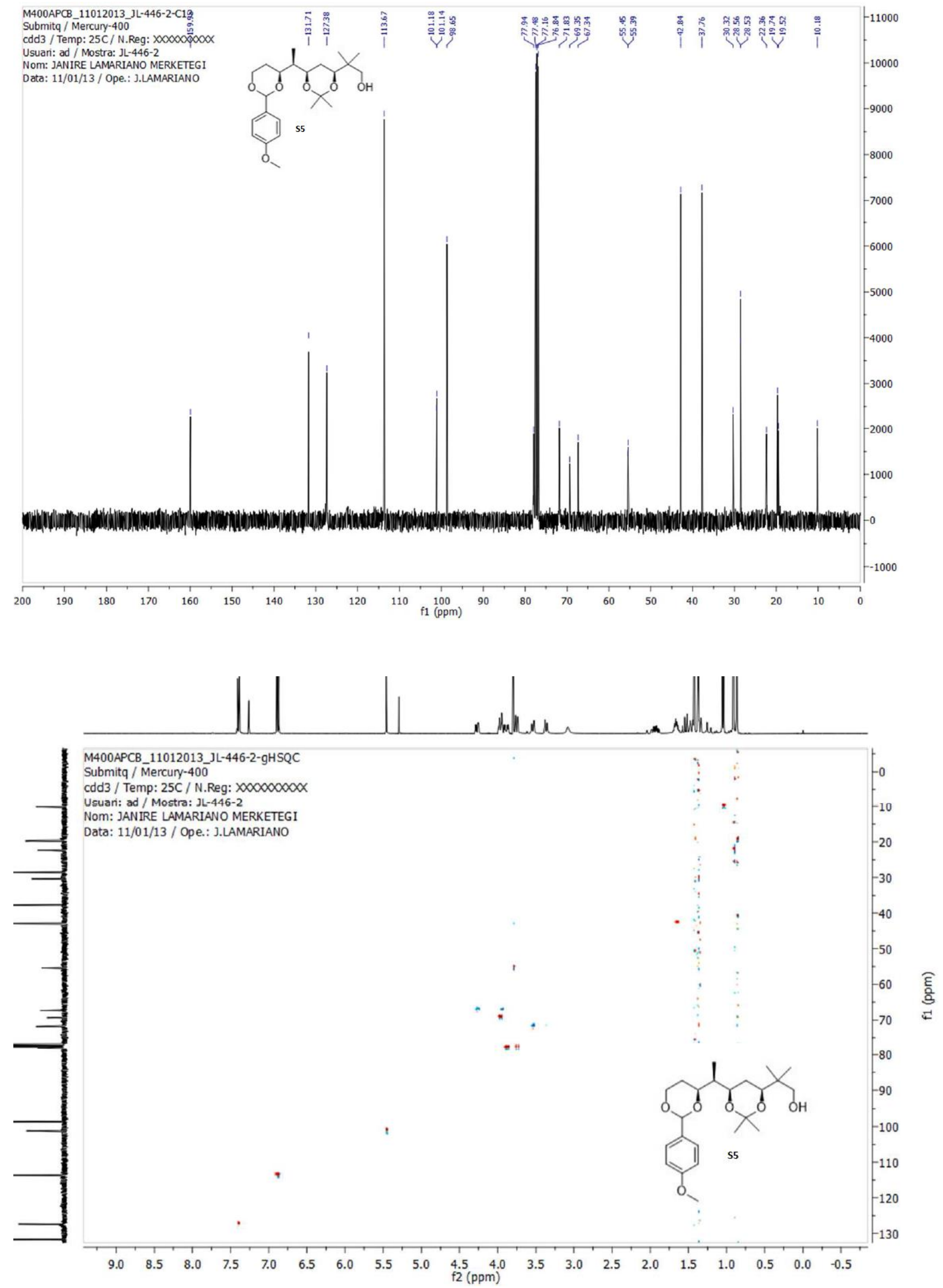

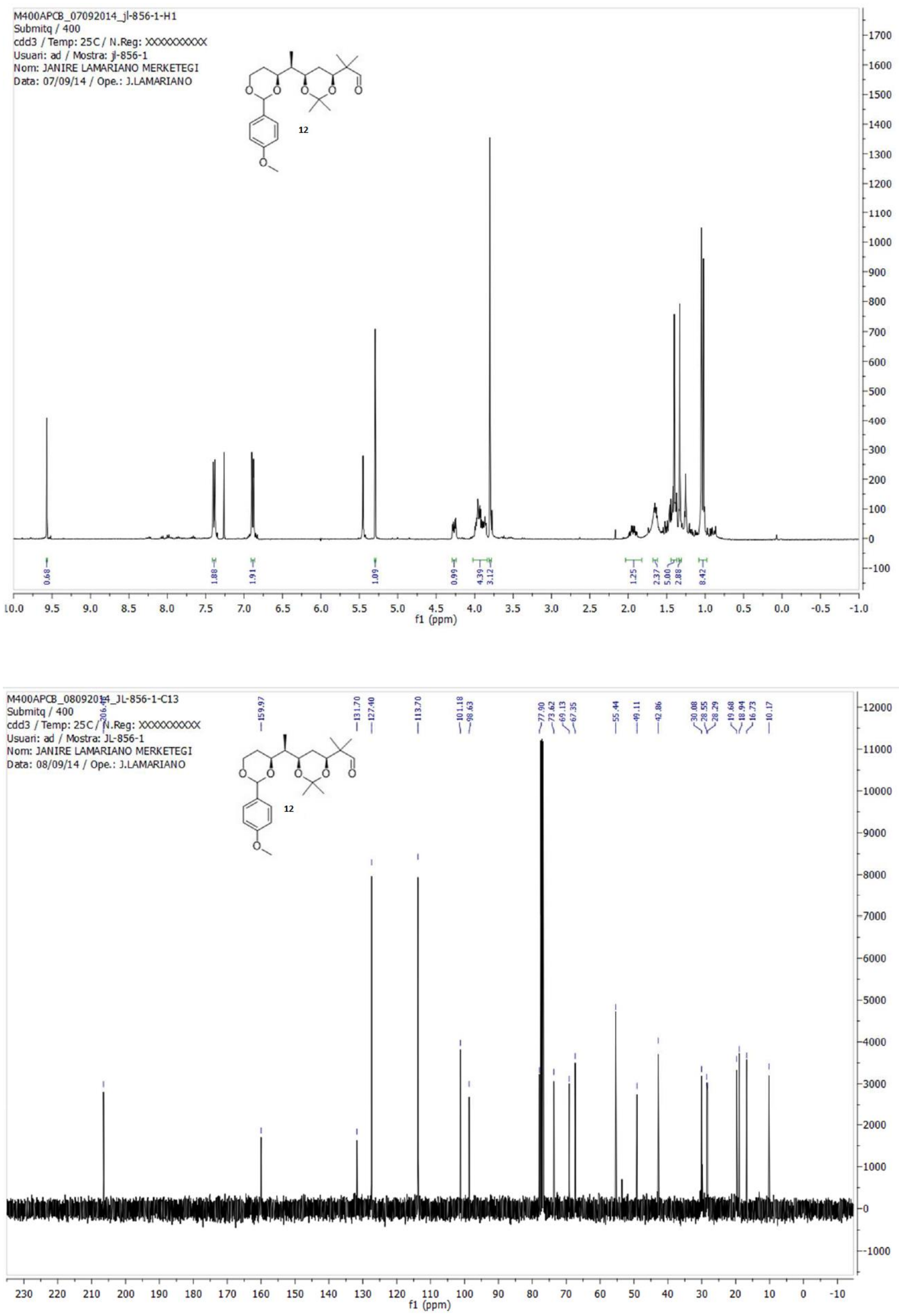

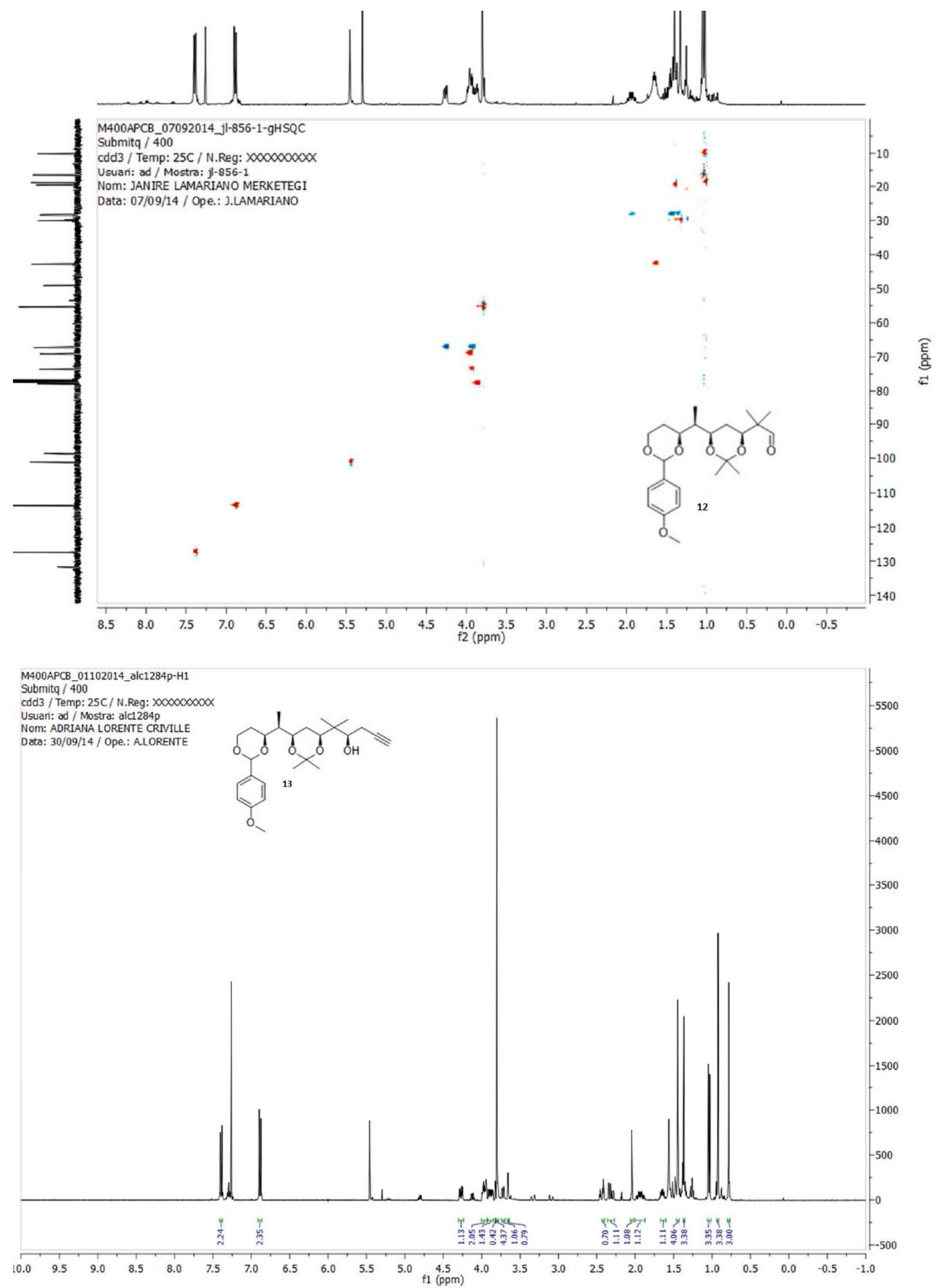

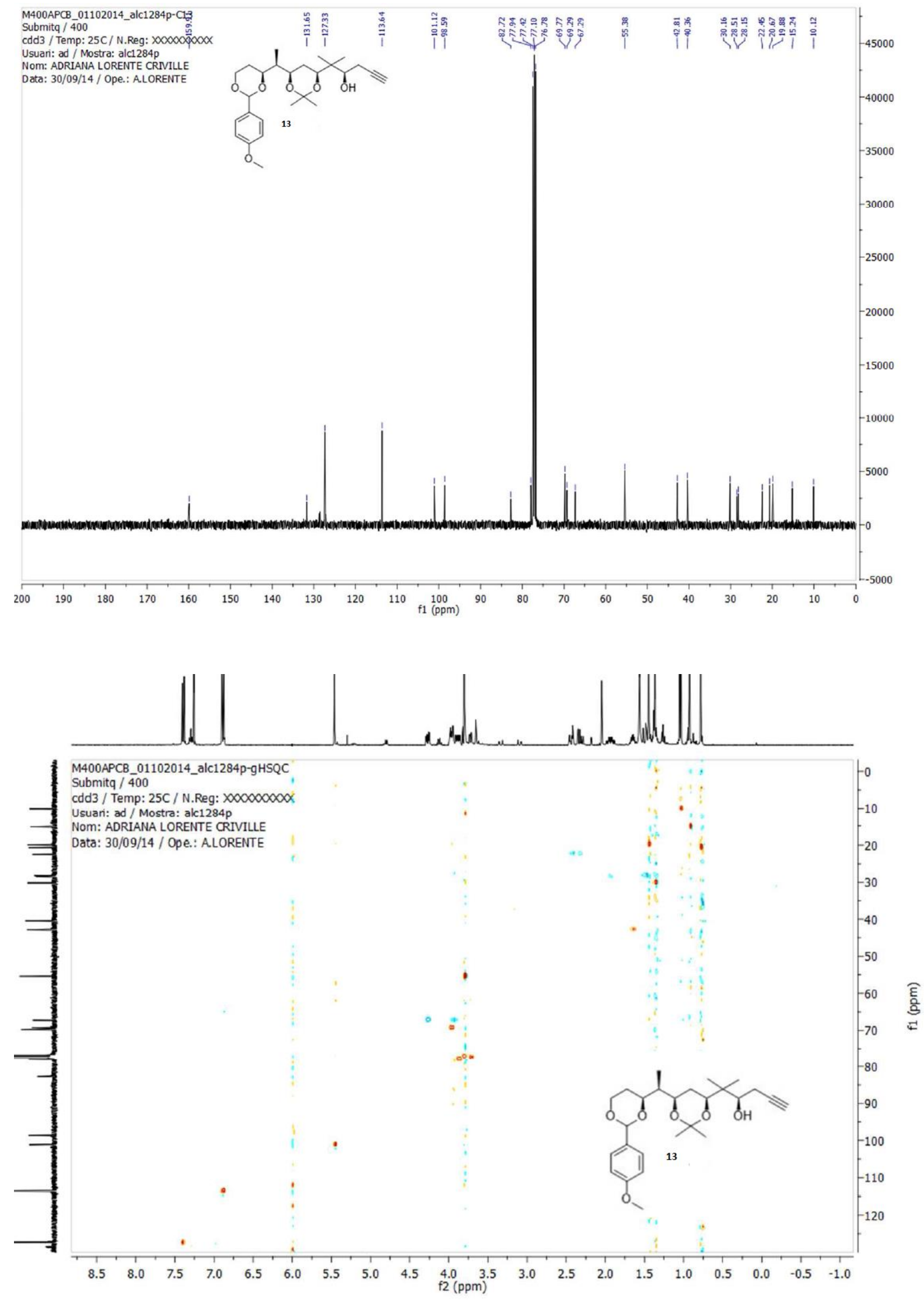

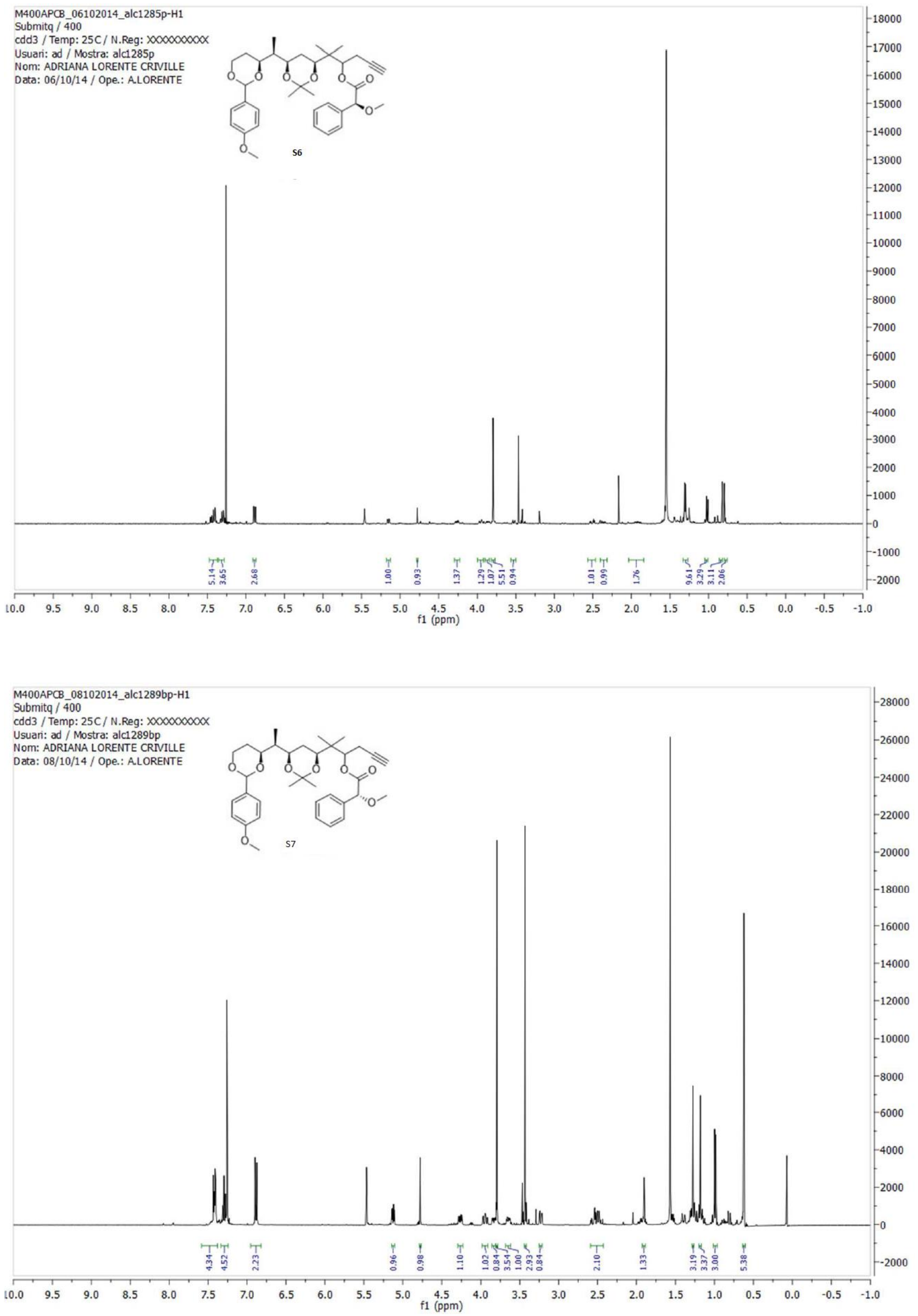

SI37 
M400APCB_19022014_JL-738-1-H1

Submitq / 400

cdcl3 / Temp: 25C/ N.Reg: 000000000

Usuari: ad / Mostra: JL-738-1

Nom: JANIRE LAMARTANO MERKETEGI

Data: 19/02/14 / Ope.: J.LAMARIANO
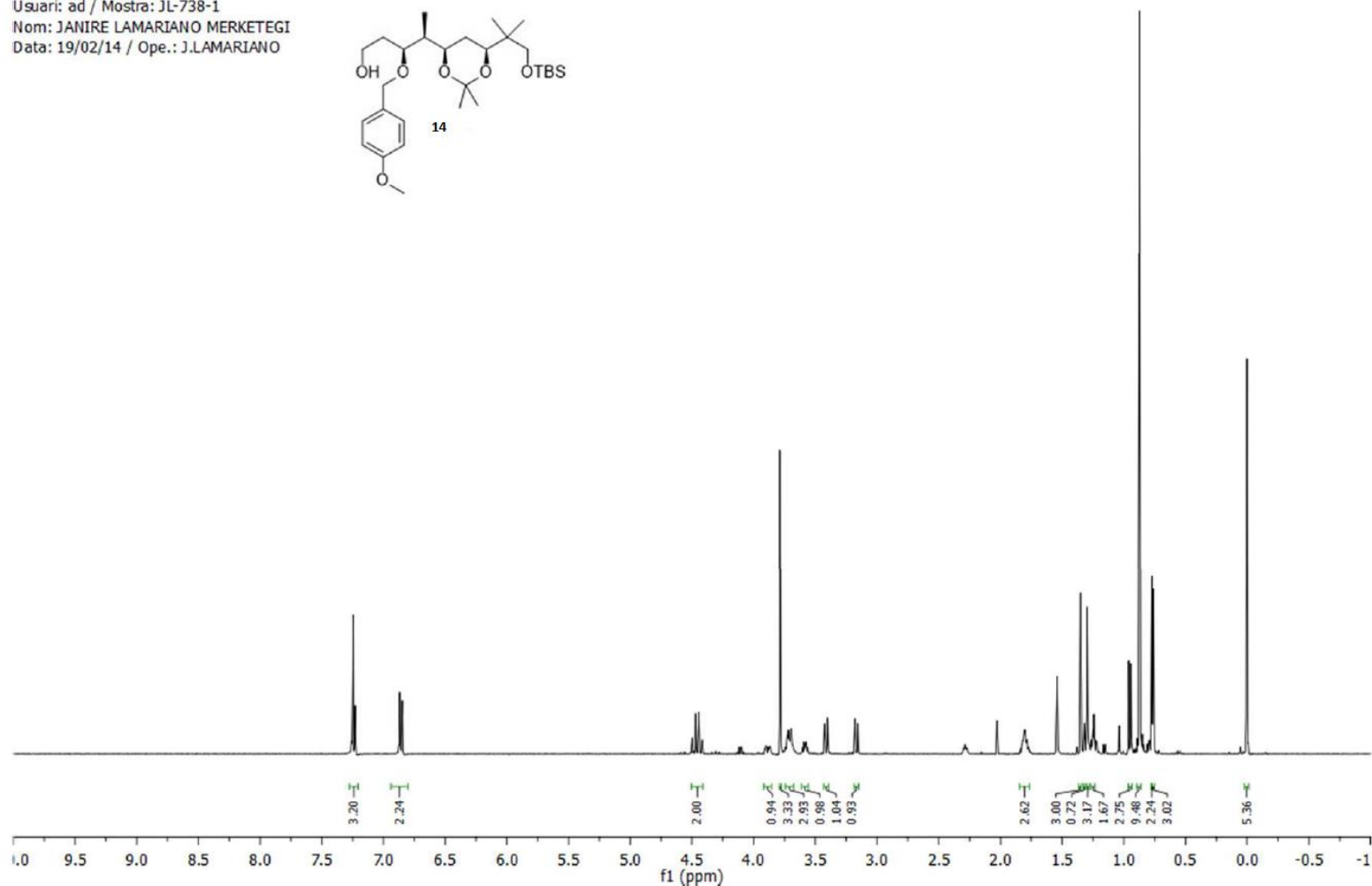

M400APCB_20022014_JL-738-1-C13\%

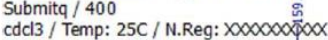
Usuari: ad / Mostra: JL-738-1

Nom: JANIRE LAMARIANO MERKETEG

Data: 19/02/14 / Ope.: J.LAMARIANO

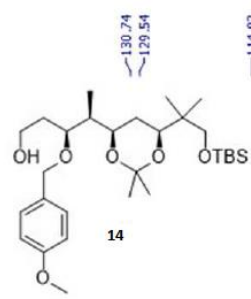

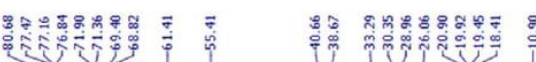

ขै

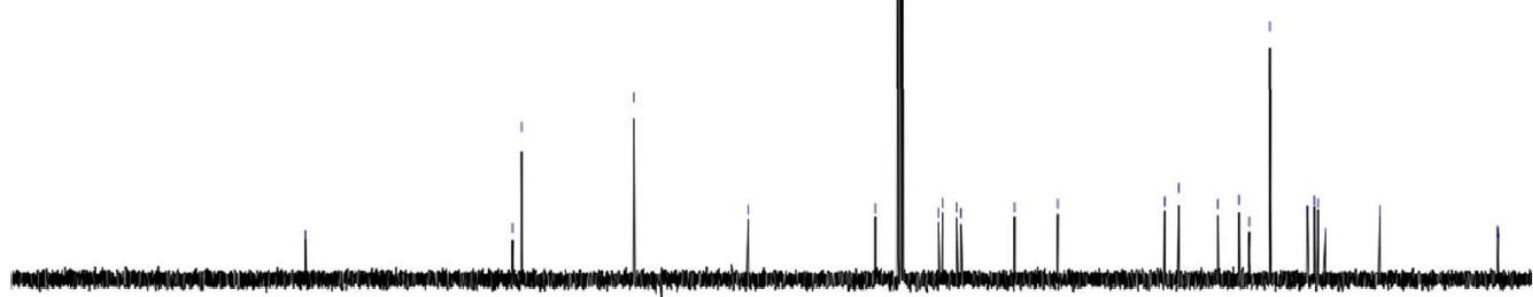

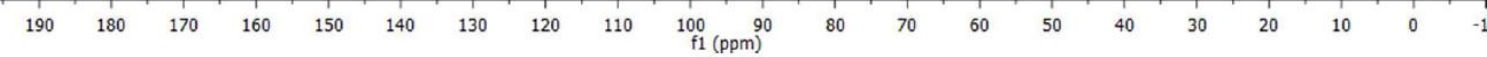




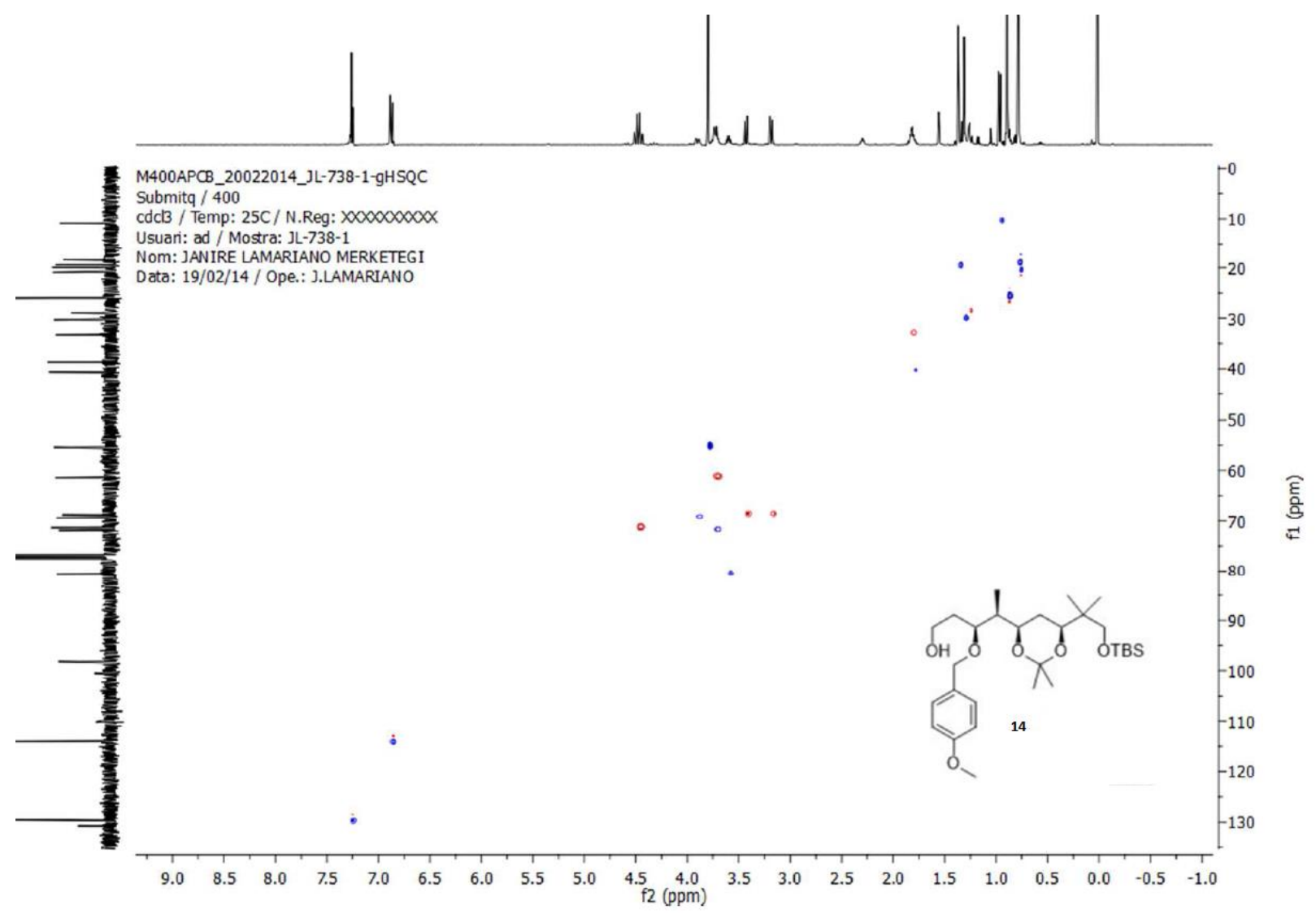

M400APCB_21022014_JL-740-1-H1

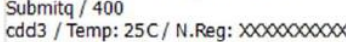

Usuari: ad / Mostra: JL-740-1

Nom: JANIRE LAMARIANO MERKETEGI Data: 21/02/14 / Ope: J.LAMARIANO
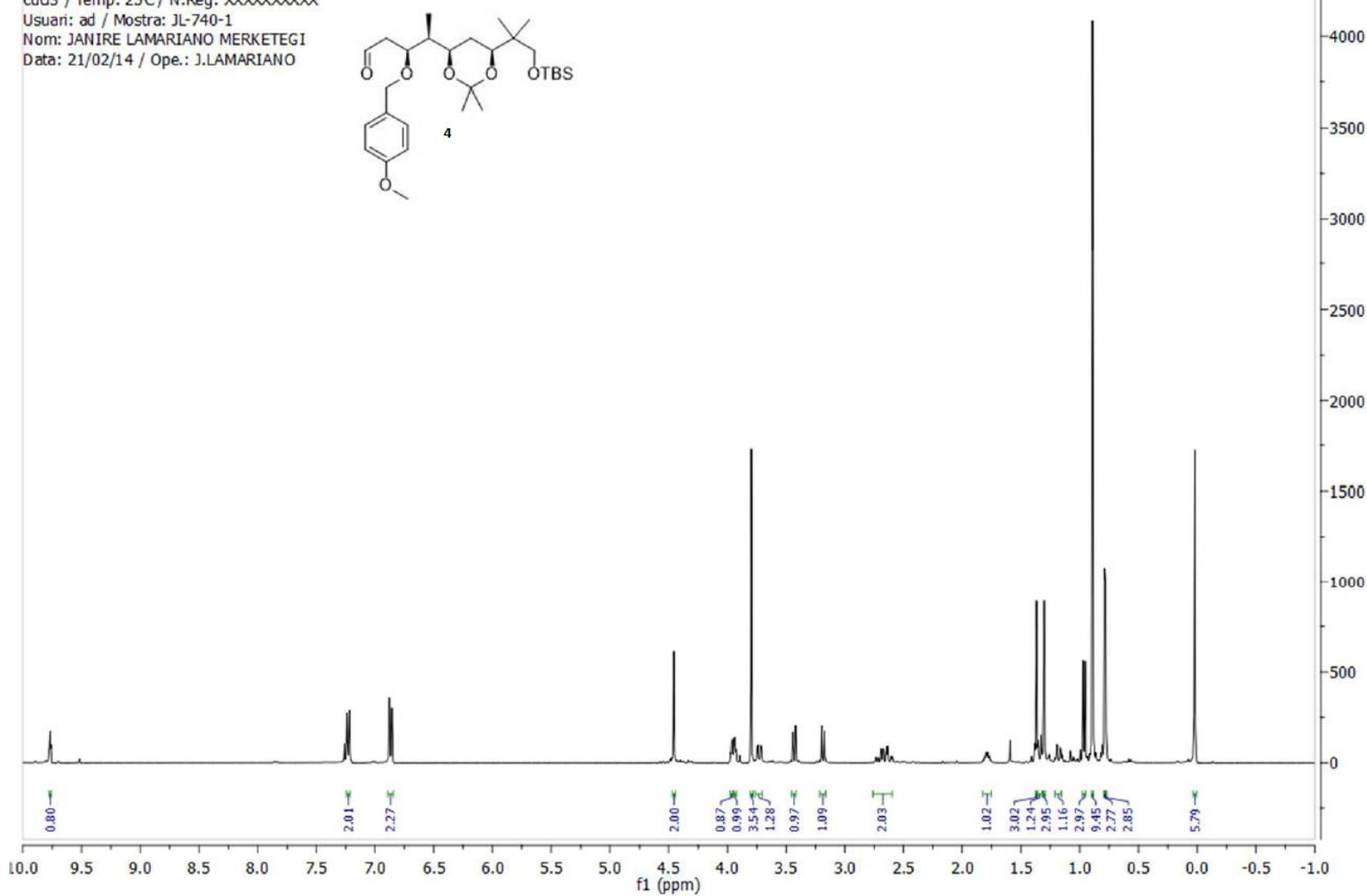

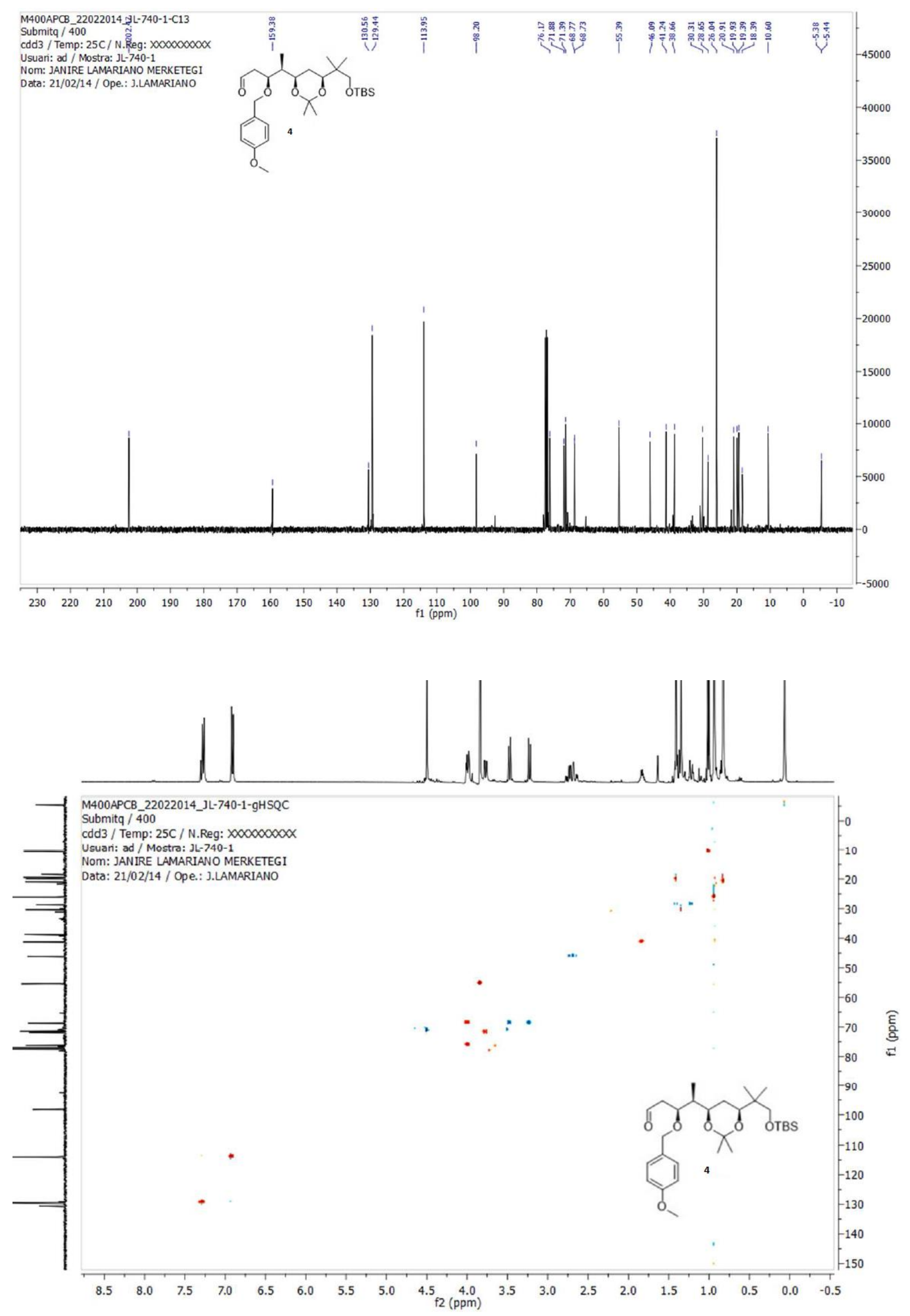
M400APCB_25042016_AGE-5.95-CR-2-H1 M400PCB / Num.Inv. AF/002630

cdcl3 / Temp: 25C / N.Reg: XXXXXXXXXX

Usuari: ad / Mostra: AGE-5.95-CR-2

i⿱

Nom: ALEJANDRO GIL ESCOLANO

Data: 25/04/16 / Ope.: A.GIL

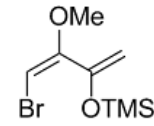

15

$-3000$

$-2800$

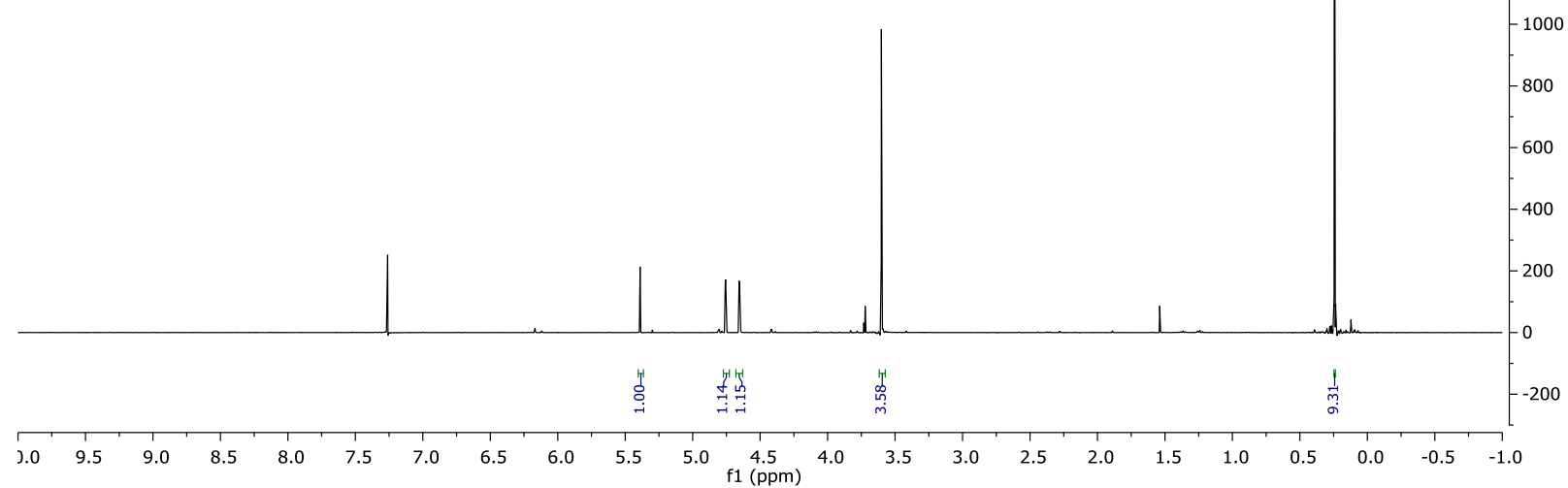
M400PCB / Num.Inv. AF/002630

suari: ad / Mostra: AGE-5.96P

Data: 26/04/16 / Ope.: A.GIL<smiles>[Y16]O[C@H](C[C@H](O)CC(=O)/C(=C\Br)OC)[C@@H](C)[C@H]1C[C@H](C(C)(C)C[OH2+])OC(C)(C)O1</smiles>

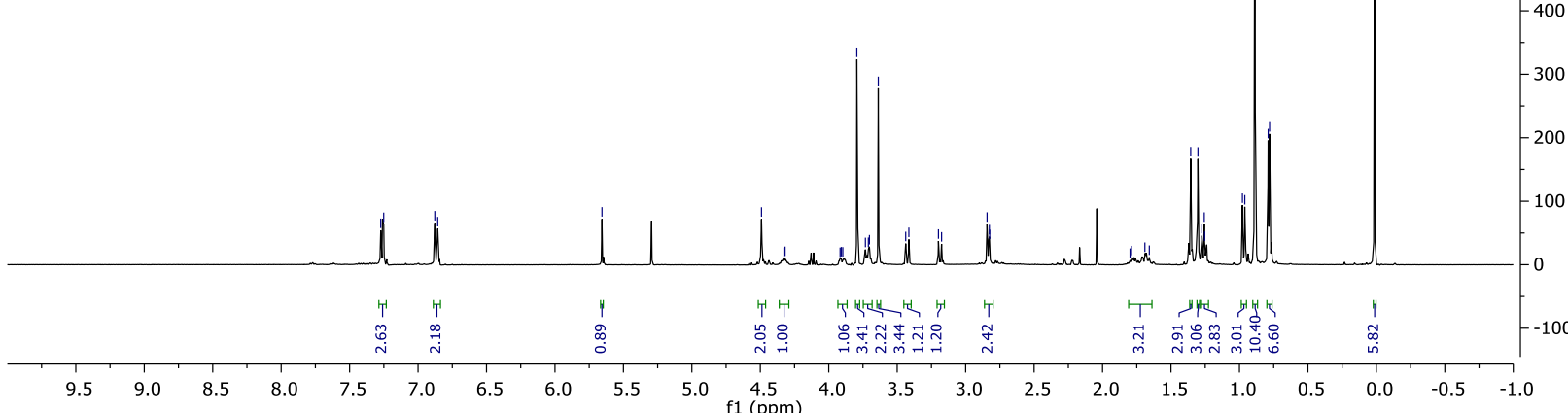


M400APCB_26042016_AGE-5_96-P-C13

M400PCB / Num.Inv. AF/002630

cdcl3 / Temp: 25C/N.Reg: XXXXXXXXXX

Usuari: ad / Mostra: AGE-5_96-P

Nom: ALEJANDRO GIL ESCOLANO

Data: 26/04/16 / Ope.: A.GIL<smiles>[Y16]O[C@H](C[C@H](O)CC(=O)/C(=C\Br)OC)[C@@H](C)[C@H]1C[C@H](C(C)(C)C[SeH])OC(C)(C)O1</smiles>
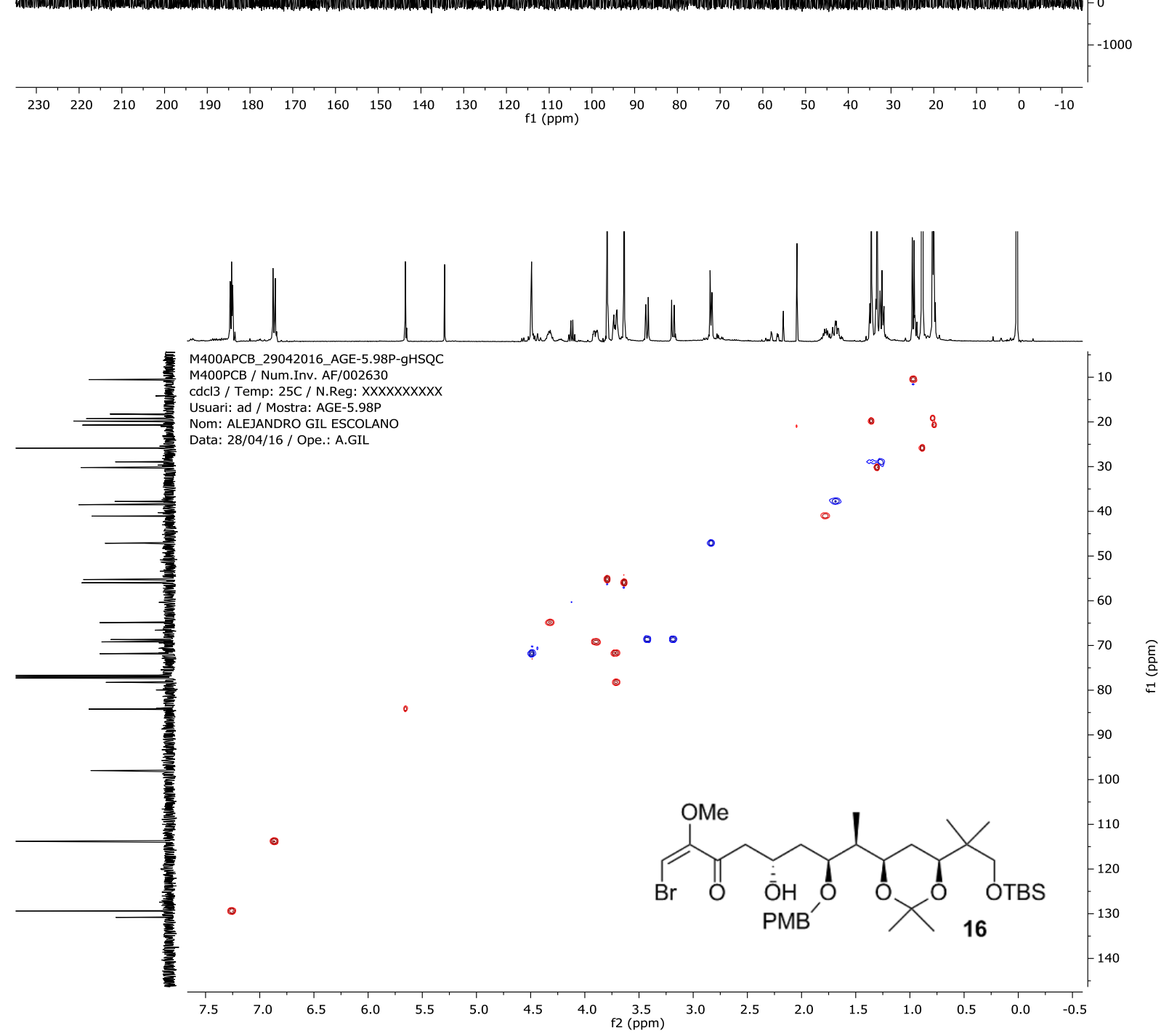


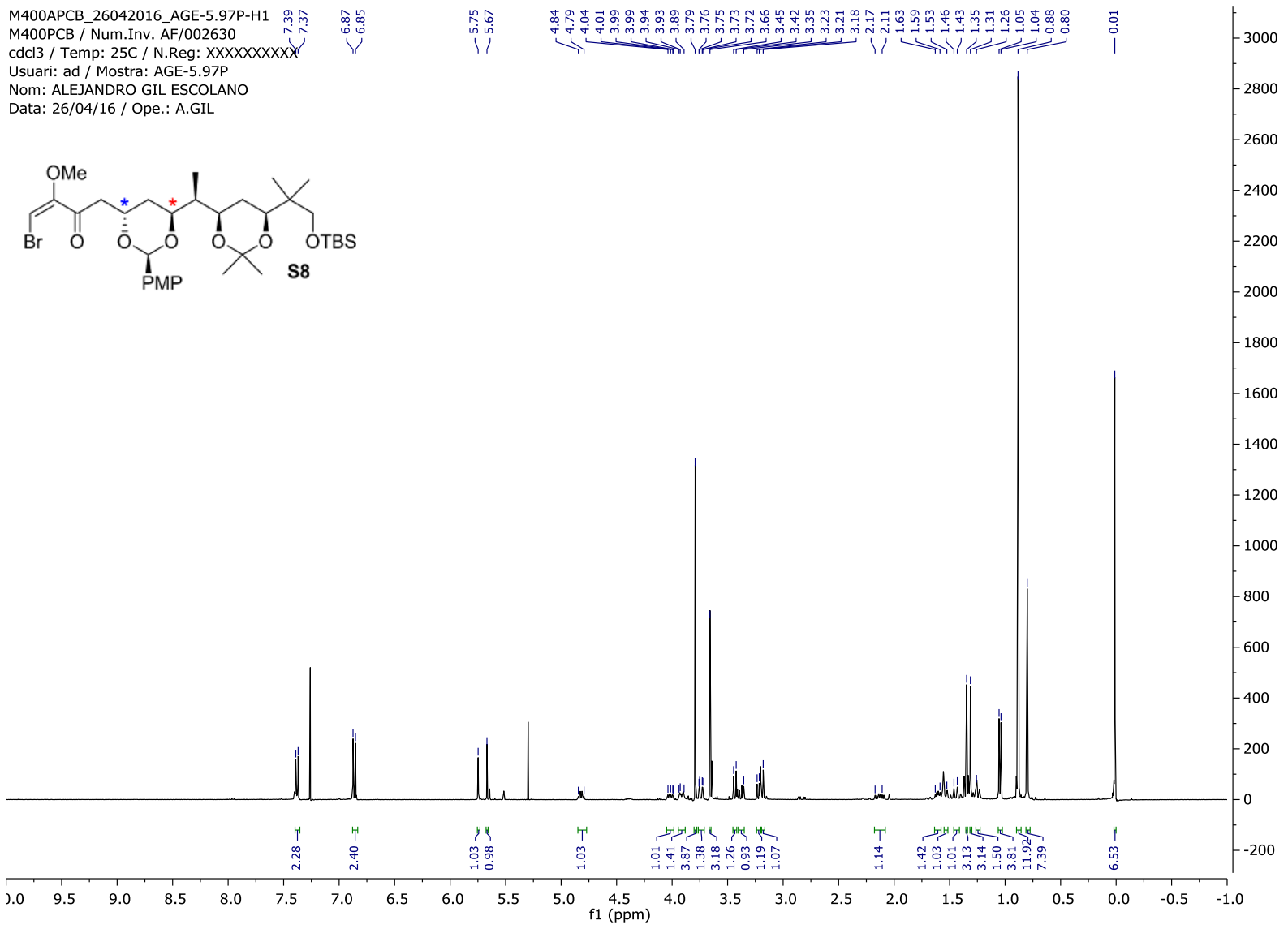

M400APCB_26042016_AGE-5.97P-C13 M40OPCB / Num.Inv. AF/002630

cdcl3 / Temp: 25C / N.Reg: XXXXXXXXXX

Usuari: ad / Mostra: AGE-5.97P

Nom: ALEJANDRO GIL ESCOLANO

Data: 26/04/16 / Ope.: A.GI
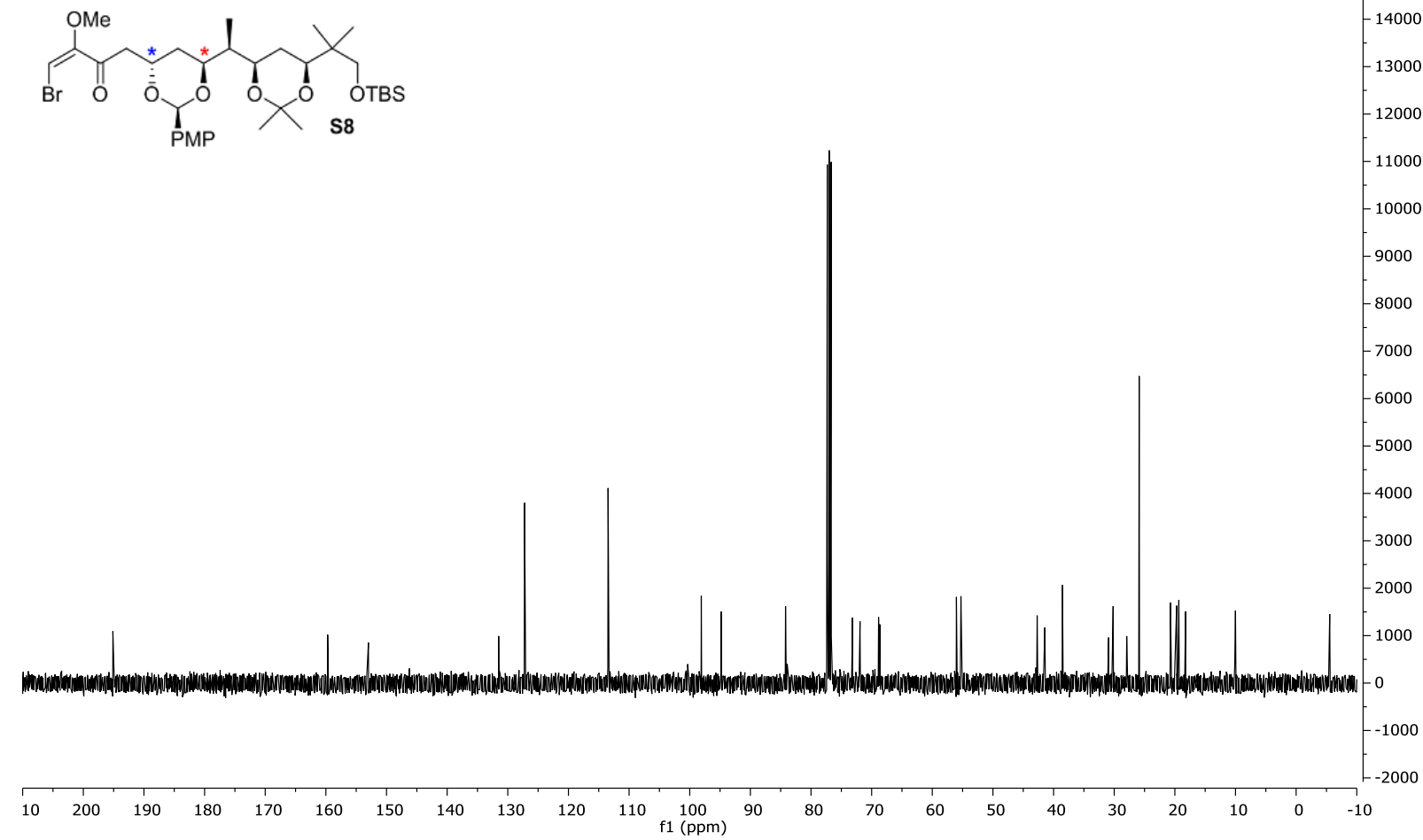

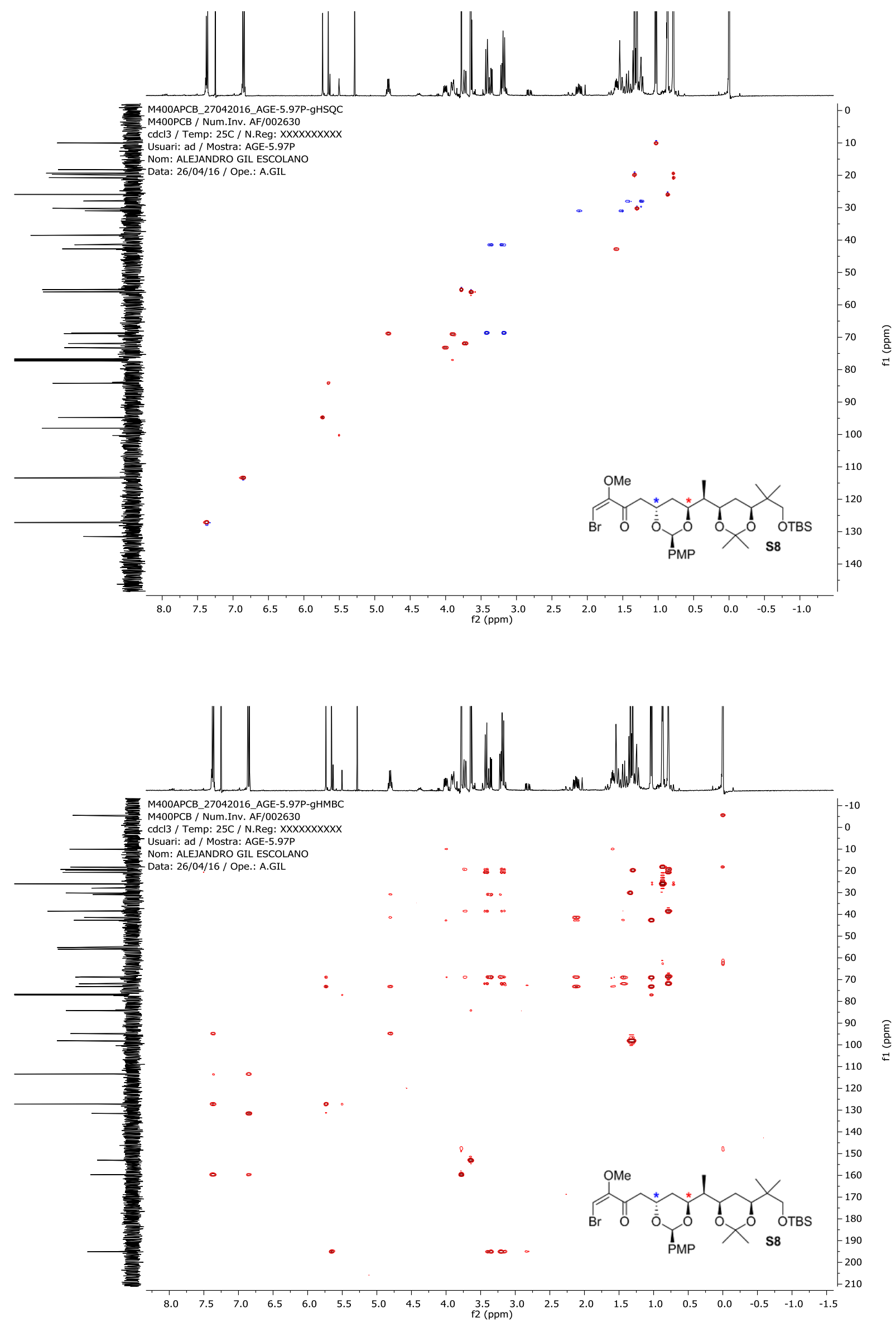


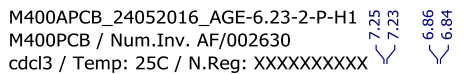

Usuari: ad / Mostra: AGE-6.23-2-P

Nom: ALEJANDRO GIL ESCOLANO

Data: 24/05/16 / Ope.: A.GIL<smiles>[Y15]O[C@H](C[C@H](CC(=O)/C(=C\Br)OC)O[As])[C@@H](C)[C@H]1C[C@H](C(C)(C)C[OH2+])OC(C)(C)O1</smiles>

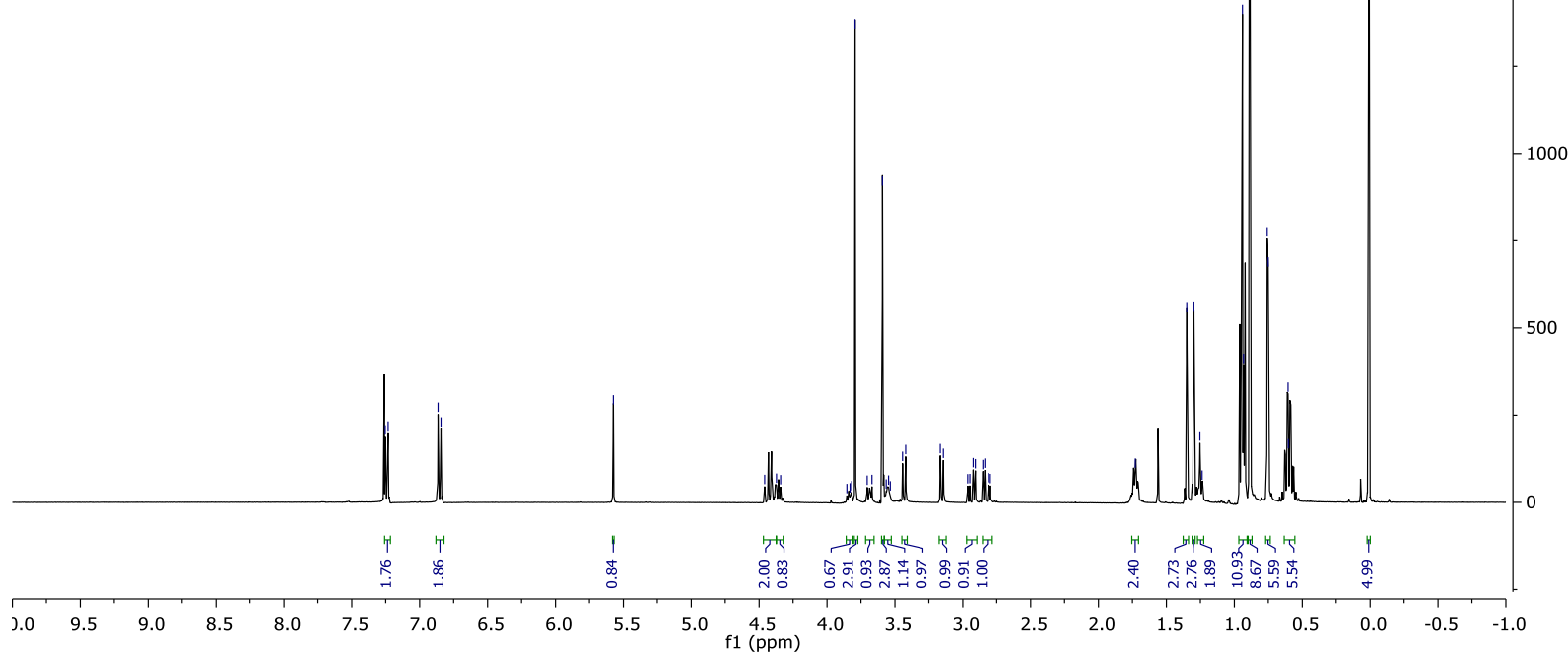

M400APCB 25052016 AGE-6.23-2-P-C13

M400PCB / Num.Inv. AF/002630

cdcl3 / Temp: 25C/N.Reg: XXXXXXXXXX

Usuari: ad / Mostra: AGE-6.23-2-P

Nom: ALEJANDRO GIL ESCOLANO

Data: 24/05/16/Ope.: A.GIL

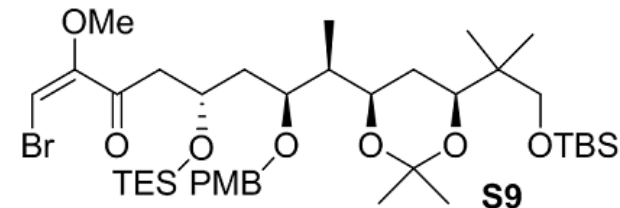

s9

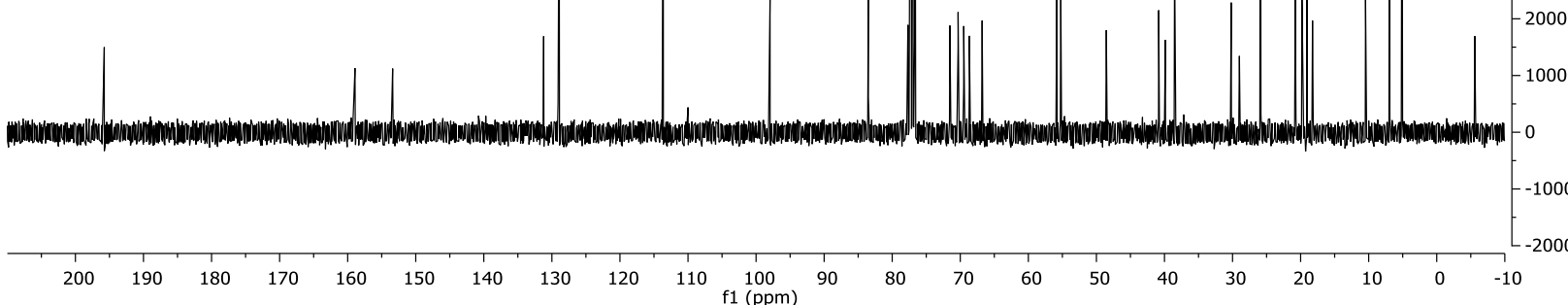




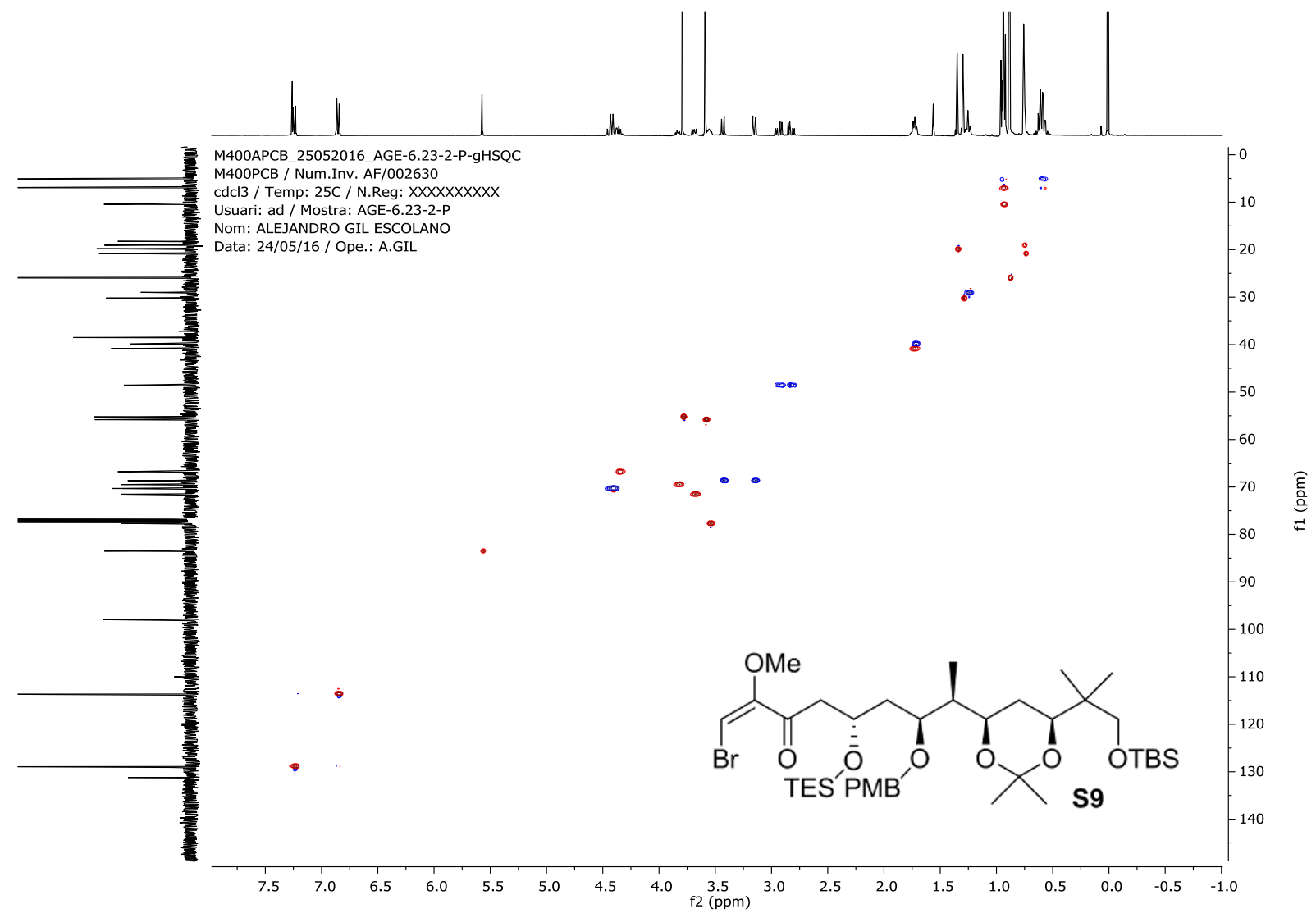

M400APCB_08062016_AGE-6.35P-H1

Usuari: ad / Mostra: AGE-6.35P

NOm: ALEJANDRO GIL ESCOLANO

Data: 08/06/16 / Ope. A.GIL

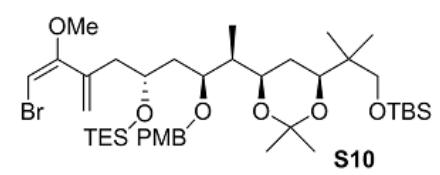

$-3600$

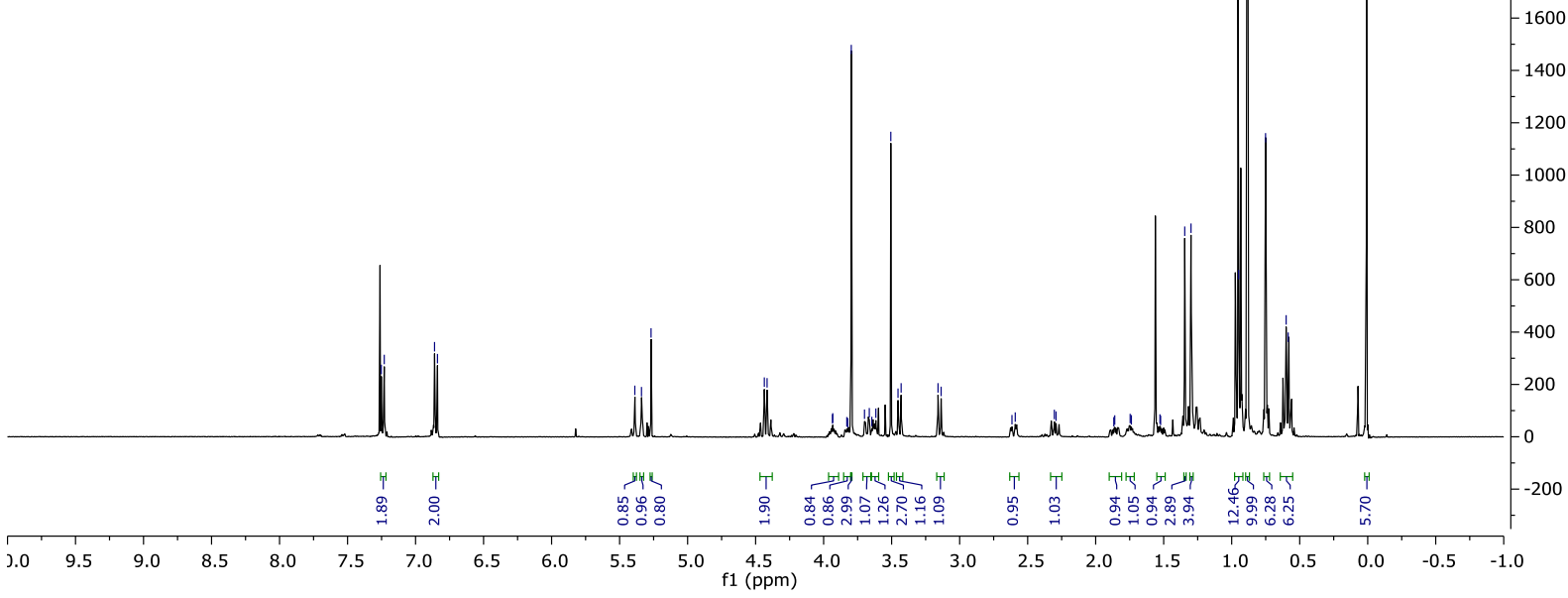


M400APCB_08062016_AGE-6.35P-C13

M400PCB / Num.Inv. AF/002630

cdcl3 / Temp: 25C/N.Reg: XXXXXXXXXX

Usuari: ad / Mostra: AGE-6.35P

Nom: ALEJANDRO GIL ESCOLANO

Data: 08/06/16 / Ope.: A.GIL

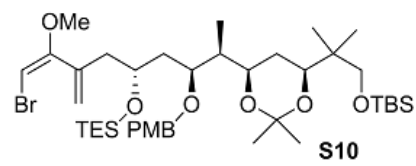
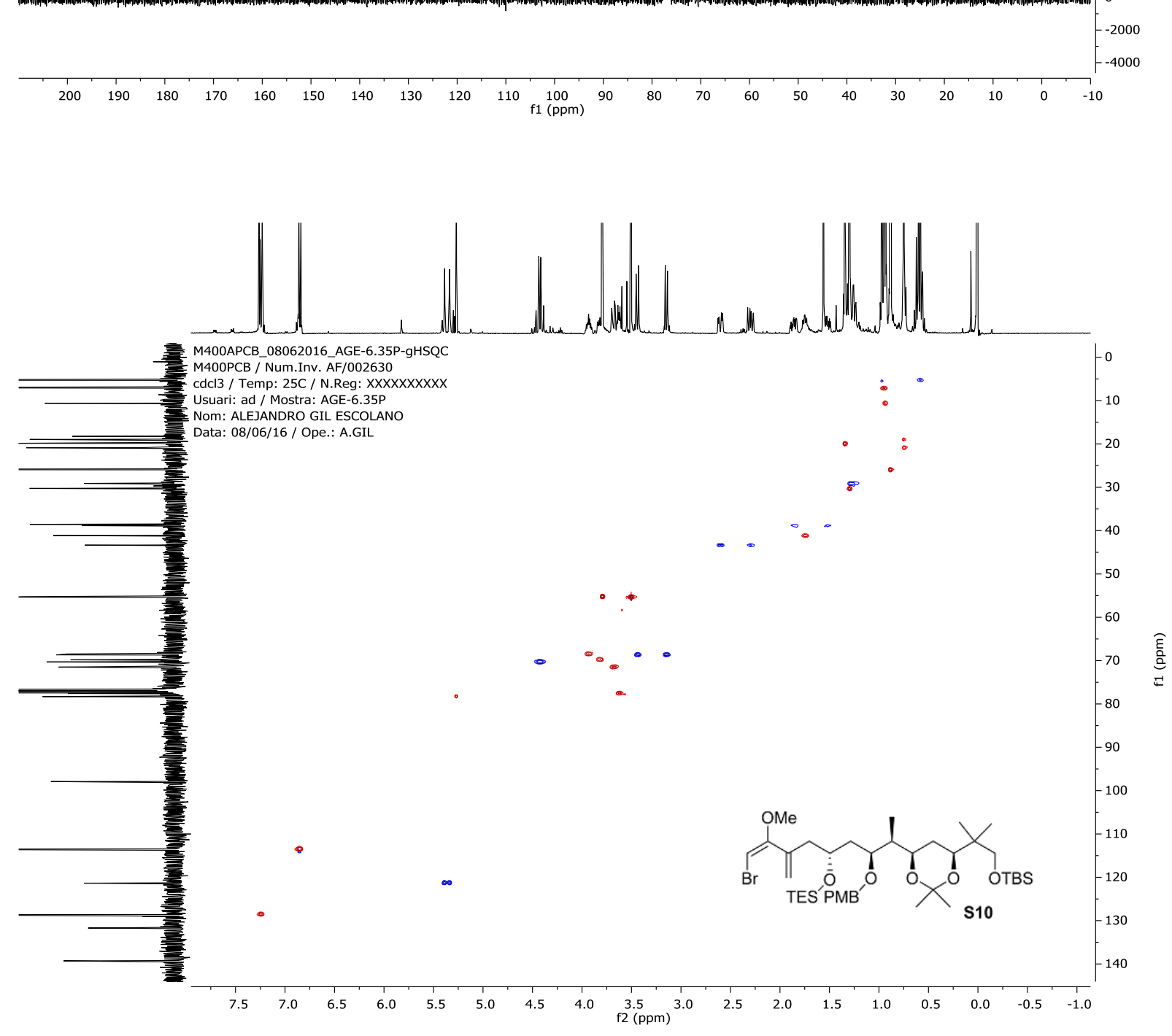


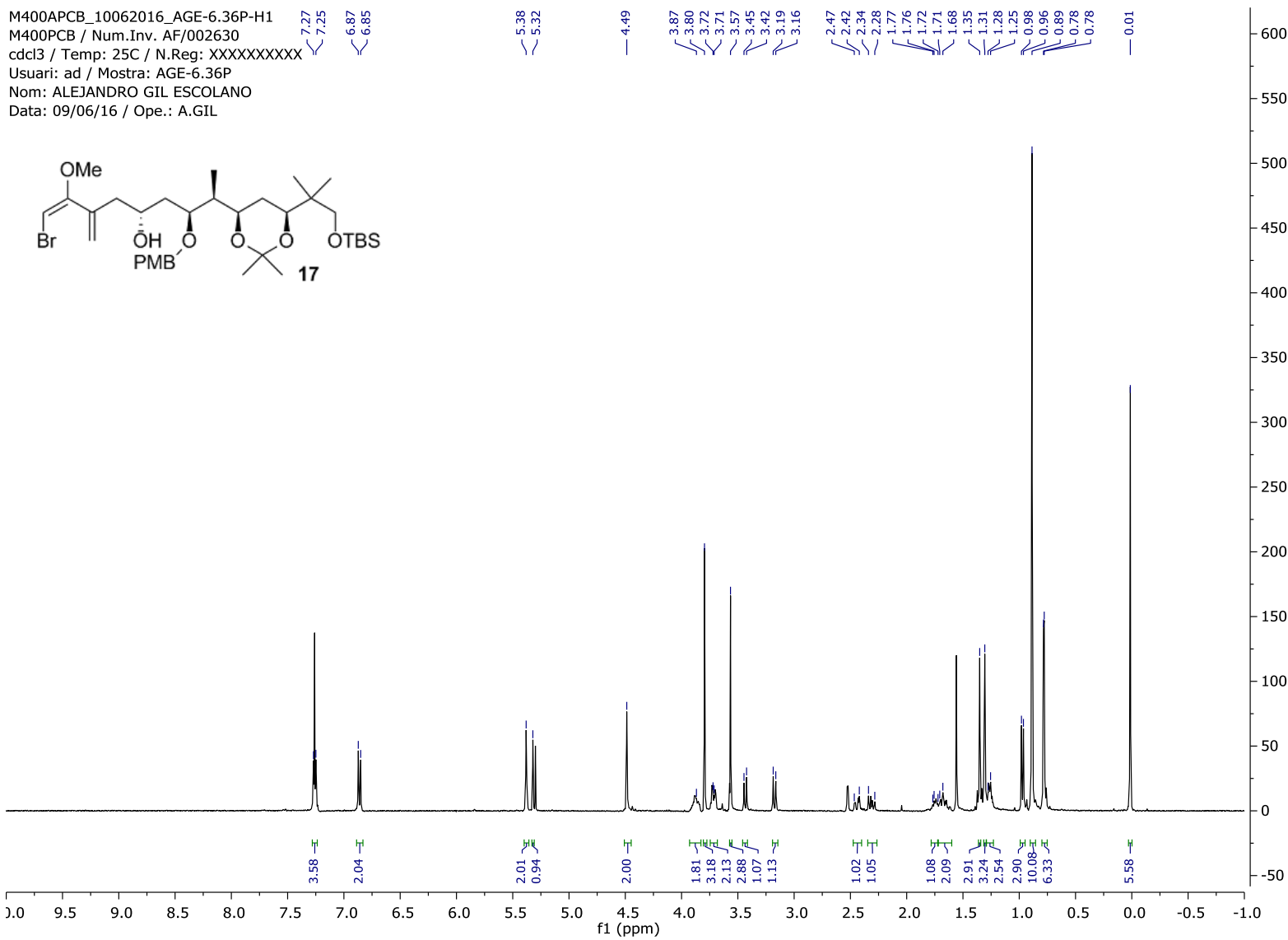

M400APCB_10062016_AGE-6.36P-C13 M400PCB / Num.Inv. AF/002630

cdc13 / Temp: 25C / N.Reg: XXXXXXXXXX

Usuari: ad/Mostra: AGE-6.36P

Nom: ALEJANDRO GIL ESCOLANO

Data: 09/06/16 / Ope.: A.GI

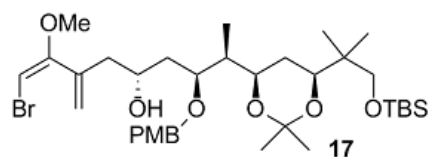

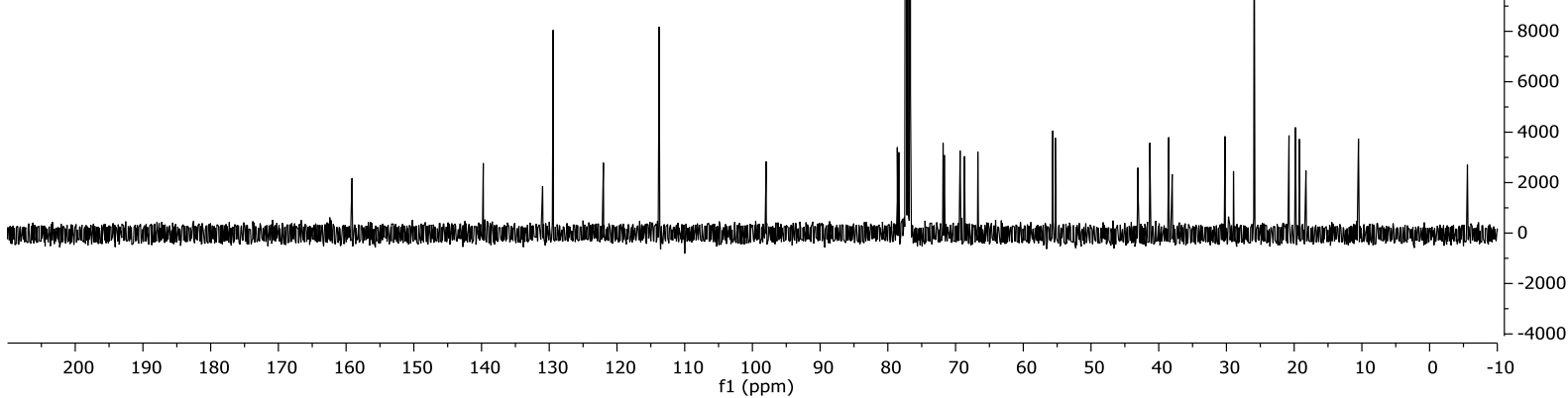



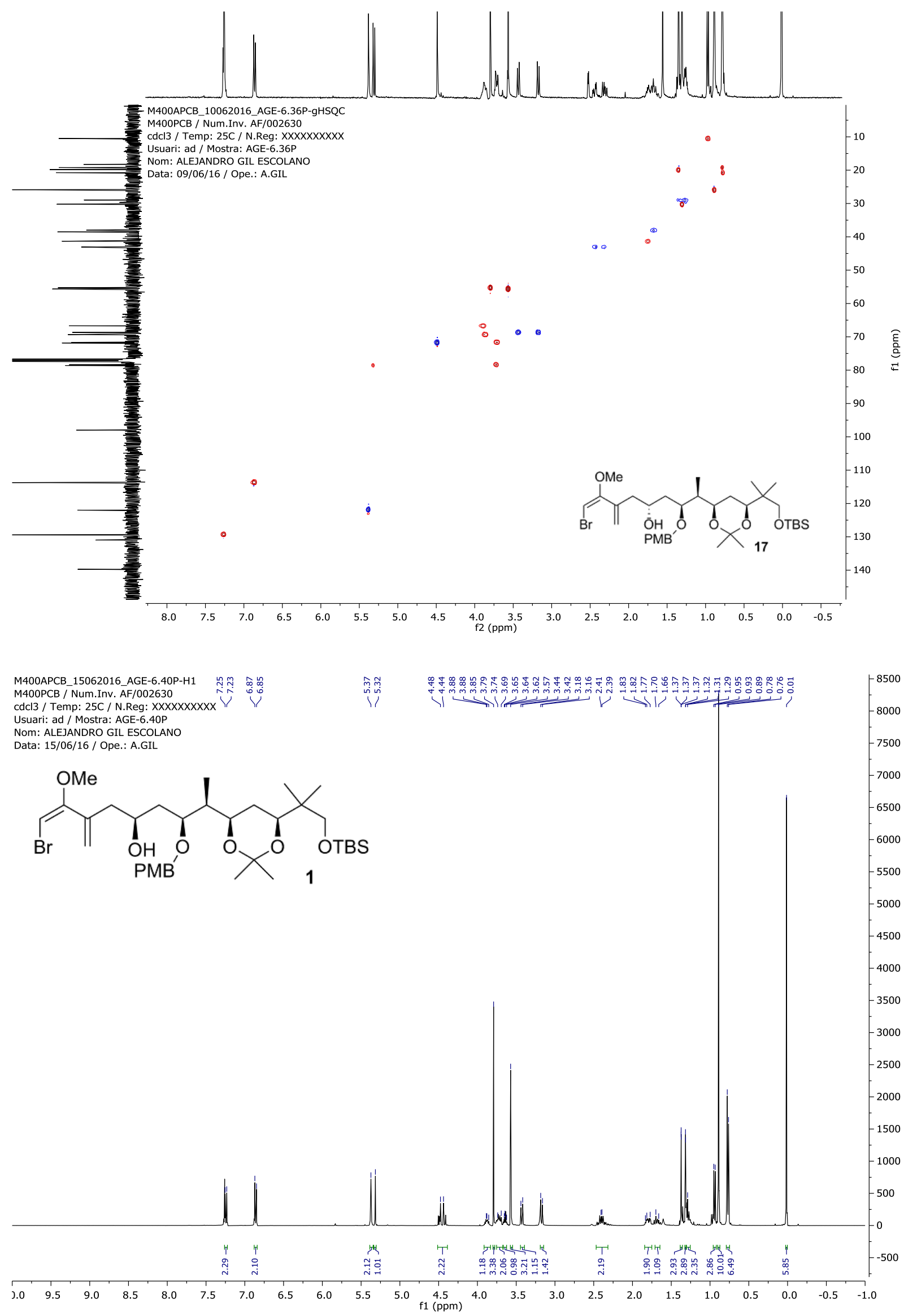
M400APCB_15062016 AGE-6.40P-C13

M400PCB / Num.Inv. AF/002630

cdc13 / Temp: 25C / N.Reg: XXXXXXXXXX

Usuari: ad / Mostra: AGE-6.40P

Nom: ALEJANDRO GIL ESCOLANO

Data: 15/06/16 / Ope.: A.GIL<smiles>[R15]O[C@H](C[C@H](O)CC(=C)/C(=C\Br)OC)[C@@H](C)[C@H]1C[C@H](C(C)(C)C[OH2+])OC(C)(C)O1</smiles>
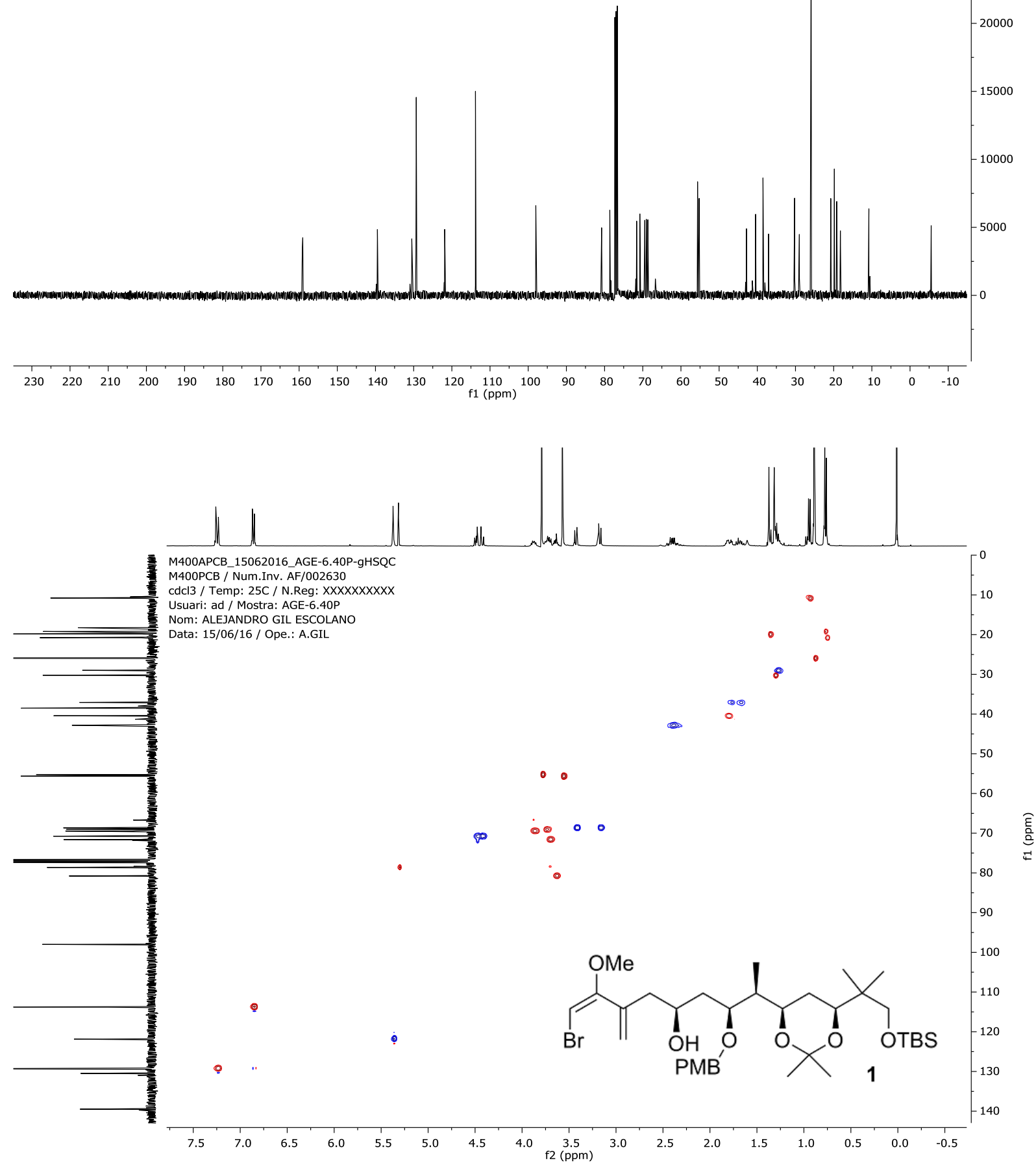


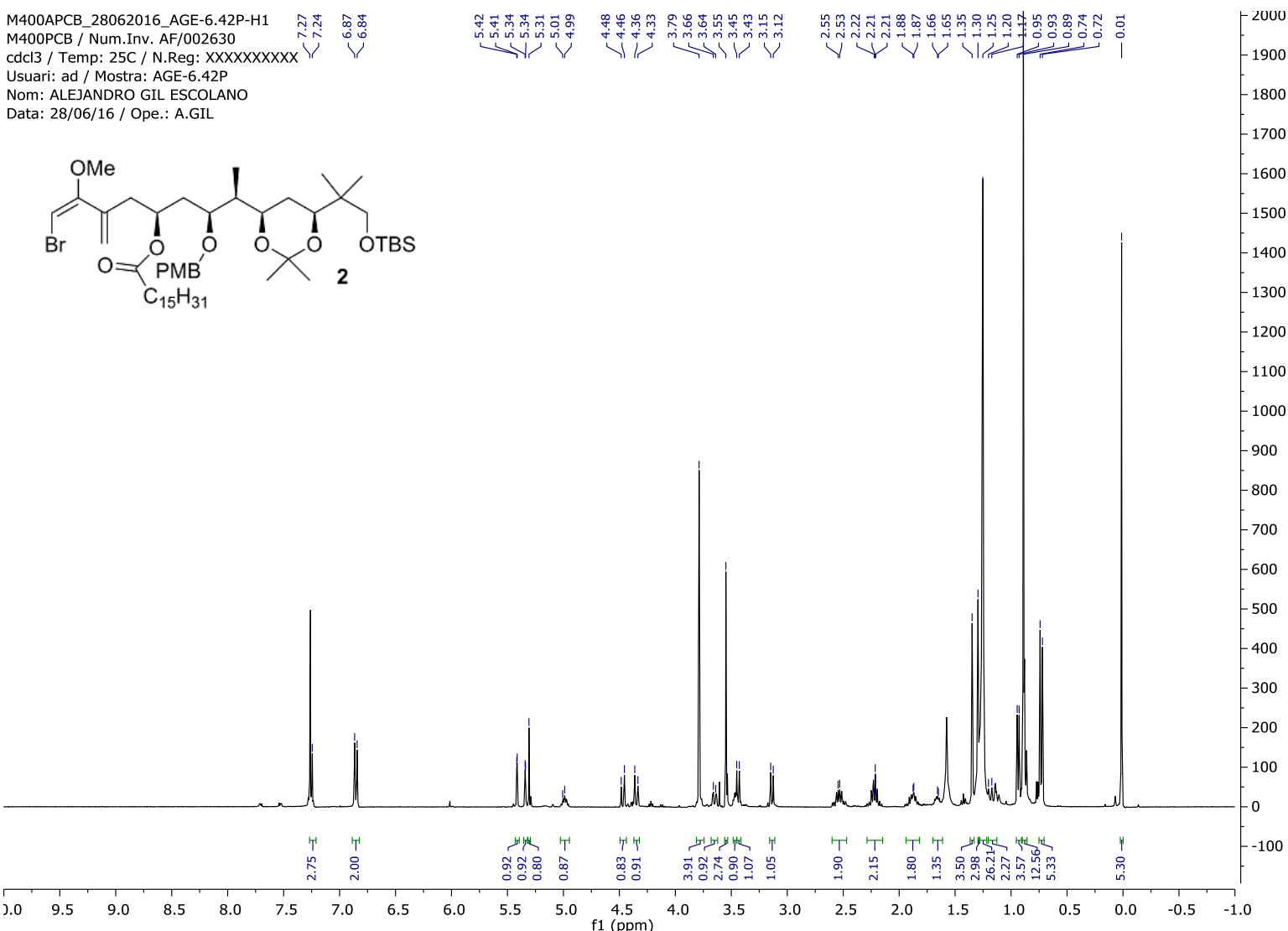

M400APCB_28062016_AGE-6.42P-C13 M400PCB / Num.Inv. AF/002630

cdcl3 / Temp: 25C / N.Reg: XXXXXXXXXXX

Usuari: ad / Mostra: AGE-6.42P

Nom: ALEJANDRO GIL ESCOLANO

Data: 28/06/16 / Ope.: A.GIL

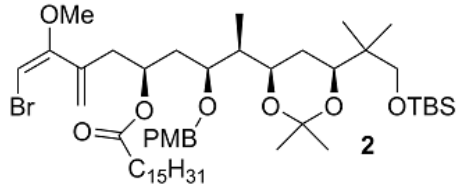

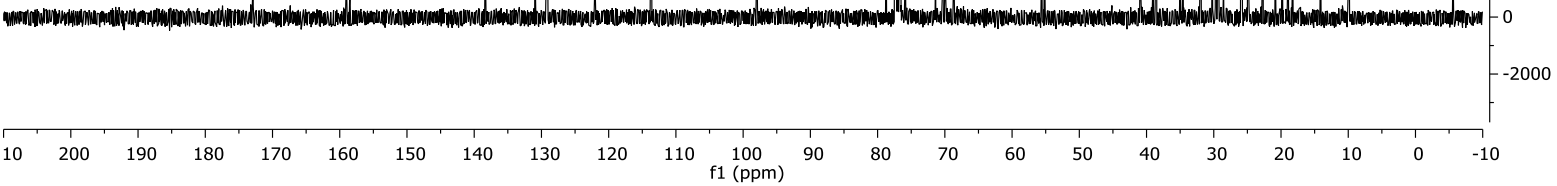



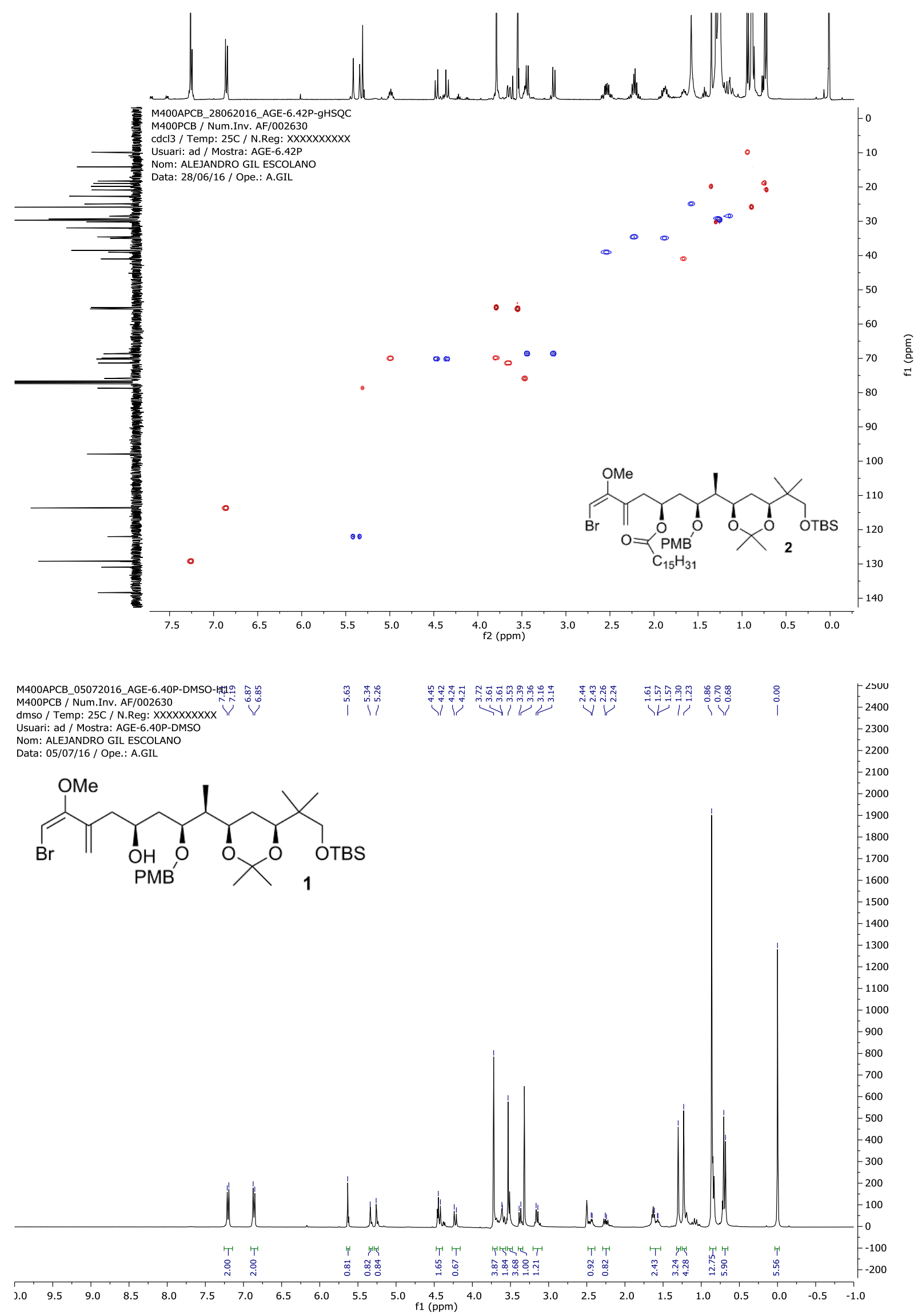
M400APCB 05072016 AGE-6 40P-DMSO-C13

M400PCB / Num.Inv. AF/002630

dmso / Temp: 25C/N.Reg: XXXXXXXXXX

Usuari: ad / Mostra: AGE-6 40P-DMSO

Nom: ALEJANDRO GIL ESCOLANO<smiles>[R15]O[C@H](C[C@H](O)CC(=C)/C(=C\Br)OC)[C@@H](C)[C@H]1C[C@H](C(C)(C)C[OH2+])OC(C)(C)O1</smiles>
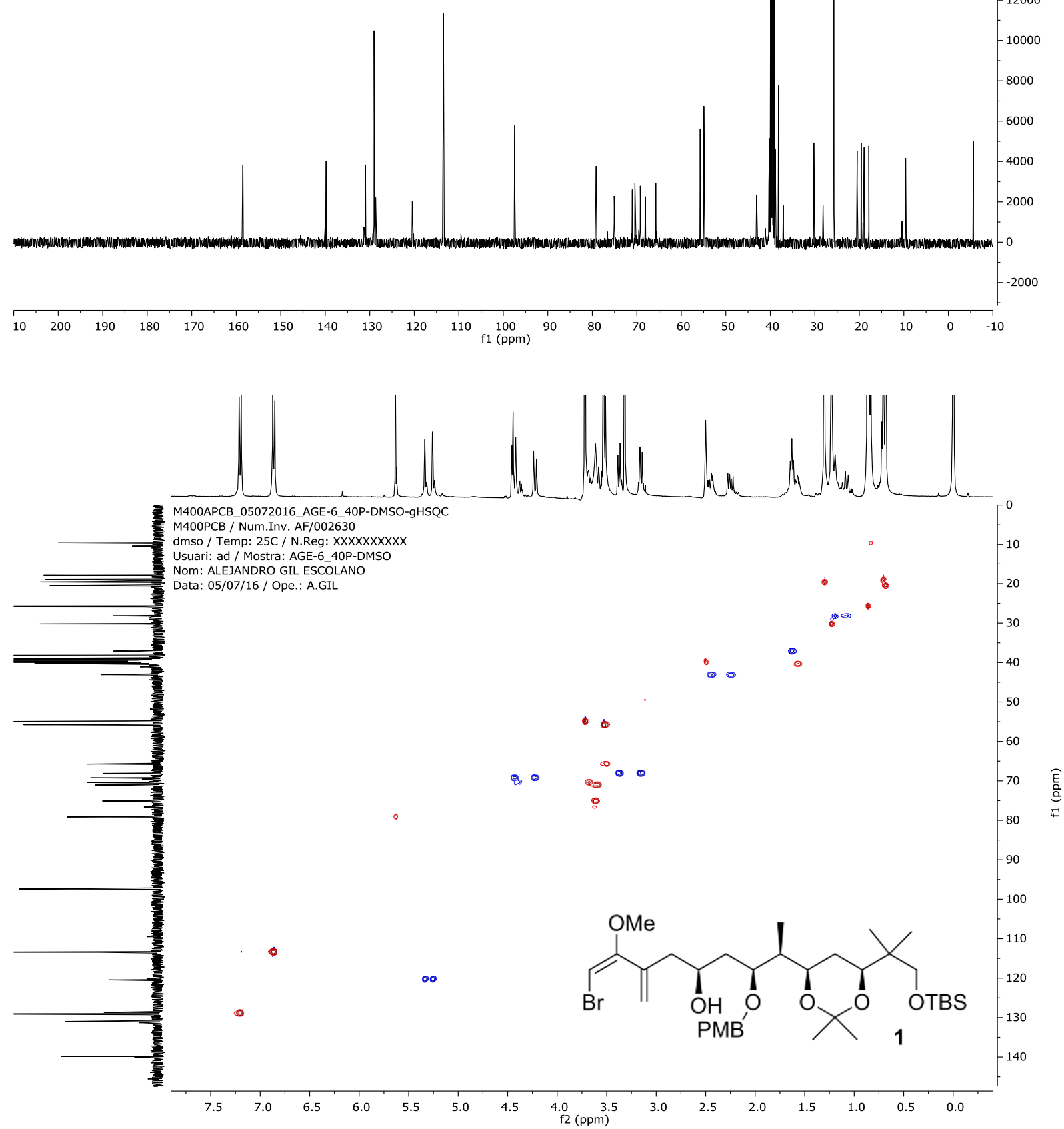


\section{References}

(1) Anderl, T.; Nicolas, L.; Münkemer, J.; Baro, A.; Sasse, F.; Steinmetz, H.; Jansen, R.; Höfle, G.; Taylor, R. E.; Laschat, S. Angew. Chemie - Int. Ed. 2011, 50 (4), 942.

(2) Pulukuri, K. K.; Chakraborty, T. K. Org. Lett. 2014, 16 (8), 2284.

(3) Gil, A.; Lamariano-Merketegi, J.; Lorente, A.; Albericio, F.; Álvarez, M. Chem. - A Eur. J. 2016, 2.

(4) Murakami, M.; Matsuda, H.; Makabe, K.; Yamaguchi, K. Tetrahedron Lett. 1991, 32, 2391.

(5) Williamson, R. T.; Boulanger, A.; Vulpanovici, A.; Roberts, M.; Gerwick, W. H. J. Org. Chem. 2002, 67, 7927. 